
Issued by Sandia National Laboratories, operated for the United States Department of Energy by Sandia Corporation.

NOTICE: This report was prepared as an account of work sponsored by an agency of the United States Government. Neither the United States Government nor any agency thereof, nor any of their employees, nor any of their contractors, subcontractors, or their employees, makes any warranty, express or implied, or assumes any legal liability or responsibility for the accuracy, completeness, or usefulness of any information, apparatus, product, or process disclosed, or represents that its use would not infringe privately owned rights. Reference herein to any specific commercial product, process, or service by trade name, trademark, manufacturer, or otherwise, does not necessarily constitute or imply its endorsement, recommendation, or favoring by the United States Government, any agency thereof or any of their contractors or subcontractors. The views and opinions expressed herein do not necessarily state or reflect those of the United States Government, any agency thereof or any of their contractors.

Printed in the United States of America. This report has been reproduced directly from the best available copy.

Available to DOE and DOE contractors from

Office of Scientific and Technical Information

PO Box 62

Oak Ridge, TN 37831

Prices available from (615) 576-8401, FTS 626-8401

Available to the public from

National Technical Information Service

US Department of Commerce

5285 Port Royal Rd

Springfield, VA 22161

NTIS price codes

Printed copy: A06

Microfiche copy: A01 


\section{DISCLAIMER}

Portions of this document may be illegible electronic image products. Images are produced from the best available original document. 
SAND91-7013

Unlimited Release

Printed October 1991

\title{
MODELLING AND FABRICATION OF HIGH-EFFICIENCY SILICON SOLAR CELLS
}

\author{
A. Rohatgi, A.W. Smith, J. Salami \\ Georgia Institute of Technology \\ School of Electrical Engineering \\ Atlanta, GA 30332
}

Sandia Contract: $42-3377$

\begin{abstract}
This report covers the research conducted on modelling and development of high-efficiency silicon solar cells during the period May 1989 to August 1990. First, considerable effort was devoted toward developing a ray-tracing program for the photovoltaic community to quantify and optimize surface texturing for solar cells. Second, attempts were made to develop a hydrodynamic model for device simulation. Such a model is somewhat slower than drift-diffusion type models like PC1D, but it can account for more physical phenomena in the device, such as hot carrier effects, temperature gradients, thermal diffusion, and lattice heat flow. In addition, Fermi-Dirac statistics have been incorporated into the model to deal with heavy doping effects more accurately. Third and final component of the research includes development of silicon cell fabrication capabilities and fabrication of high-efficiency silicon cells.
\end{abstract}

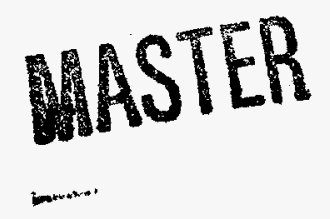




\section{TABLE OF CONTENTS}

TABLE OF CONTENTS $\ldots \ldots \ldots \ldots \ldots \ldots \ldots \ldots \ldots \ldots \ldots \ldots$

LIST OF FIGURES $\ldots \ldots \ldots \ldots \ldots \ldots \ldots \ldots \ldots \ldots \ldots \ldots \ldots \ldots$

LIST OF TABLE $\ldots \ldots \ldots \ldots \ldots \ldots \ldots \ldots \ldots \ldots \ldots \ldots \ldots \ldots$

GLOSSARY OF MATHEMATICAL TERMS $\ldots \ldots \ldots \ldots \ldots \ldots \ldots \ldots$

1. $\underline{\text { SUMMARY } \ldots \ldots \ldots \ldots \ldots \ldots \ldots \ldots \ldots \ldots \ldots \ldots \ldots \ldots \ldots \ldots \ldots}$

2. MODELLING OF LIGHT TRAPPING IN SOLAR CELLS BY TEXTURED

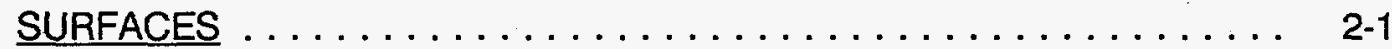

2.1 History of the Analysis of Textured Solar Cells $\ldots \ldots \ldots \ldots$ 2-1

2.2. Development of Ray Tracing Model: TEXTURE . . . . . . . . . . 2-10

Mathematics of Analysis $\ldots \ldots \ldots \ldots \ldots \ldots . \ldots \ldots$

Intersection of Lines and Planes $\ldots \ldots \ldots \ldots \ldots . \ldots . . \ldots 2-10$

Refraction of Light . . . . . . . . . . . . . . . . . . . 2-12

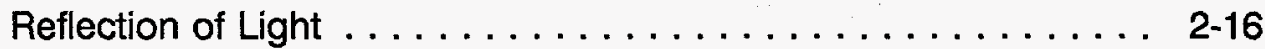

"Constrained" Volume ... . . . . . . . . . . . . . . . . . . 2-18

Minimum Distance . . . . . . . . . . . . . . . . . . . 2.25

Program Structure $\ldots \ldots \ldots \ldots \ldots \ldots \ldots . \ldots \ldots$ 2-28

Program Flow . . . . . . . . . . . . . . . . . . 2-29

Program Input $\ldots \ldots \ldots \ldots \ldots \ldots \ldots \ldots \ldots \ldots . \ldots \ldots$ 2-32

Program Output $\ldots \ldots \ldots \ldots \ldots \ldots \ldots \ldots \ldots \ldots$ 2-35 
3. THE HYDRODYNAMIC MODEL FOR SEMICONDUCTOR DEVICE

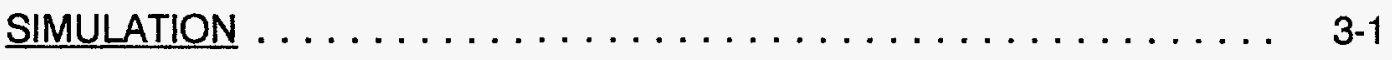

3.1. Pros and Cons of Hydrodynamic Model $\ldots \ldots \ldots \ldots \ldots \ldots$ 3-1

3.2. Development of Transport Model $\ldots \ldots \ldots \ldots \ldots \ldots \ldots \ldots$. 3-5

Assumptions of BTE $\ldots \ldots \ldots \ldots \ldots \ldots \ldots \ldots \ldots \ldots \ldots \ldots \ldots$

Particle Balance Equation $\ldots \ldots \ldots \ldots \ldots \ldots \ldots \ldots \ldots \ldots$

Particle Flux Balance Equation . . . . . . . . $\ldots \ldots \ldots \ldots$.

Energy Balance Equation $\ldots \ldots \ldots \ldots \ldots \ldots \ldots \ldots$ 3-11

Energy Flux Balance Equation $\ldots \ldots \ldots \ldots \ldots \ldots \ldots \ldots \ldots$

3.3. Potential Application to Solar cells, Thyristors, and HBT's ...... 3-18

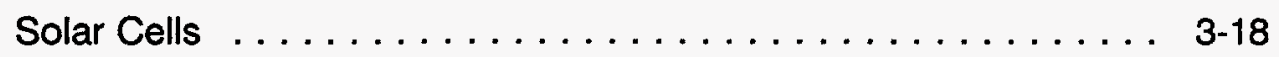

Thyristors $\ldots \ldots \ldots \ldots \ldots \ldots \ldots \ldots \ldots \ldots \ldots \ldots \ldots \ldots \ldots$ 3-19

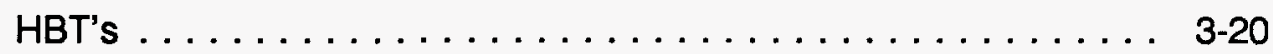

3.4. Preliminary Results of Hydrodynamic Model $\ldots \ldots \ldots \ldots \ldots .3-21$

4. RE-EVALUATION OF THE DERIVATIVES OF THE HALF ORDER FERMI

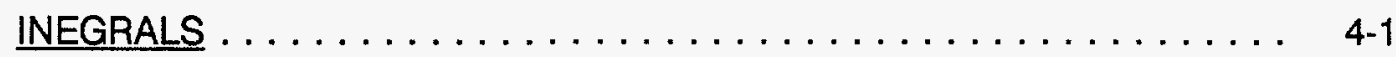

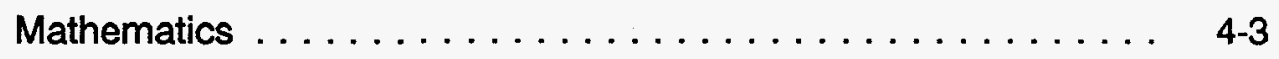

Evaluation of Integrals $\ldots \ldots \ldots \ldots \ldots \ldots \ldots \ldots \ldots \ldots \ldots \ldots \ldots$

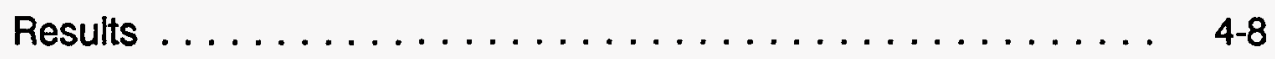

5. SILICON SOLAR CELL FABRICATION $\ldots \ldots \ldots \ldots \ldots \ldots \ldots \ldots \ldots$ 5-1

5.1 Process Development $\ldots \ldots \ldots \ldots \ldots \ldots \ldots \ldots \ldots \ldots$ 5-1

TCA Gettering of the Furnace Tubes $\ldots \ldots \ldots \ldots \ldots \ldots \ldots$ 5-1 
Oxidation of Silicon $\ldots \ldots \ldots \ldots \ldots \ldots \ldots \ldots \ldots \ldots \ldots \ldots$

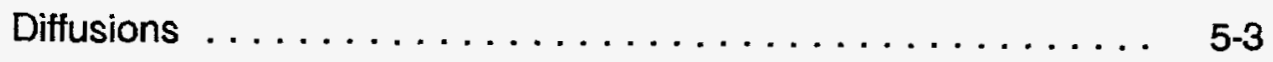

Texturing and Thinning Processes $\ldots \ldots \ldots \ldots \ldots \ldots . \ldots \ldots$

5.2 DEVELOPMENT OF ANTIREFLECTION COATINGS: MODELLING AND EXPERIMENT $\ldots \ldots \ldots \ldots \ldots \ldots \ldots \ldots \ldots \ldots \ldots \ldots$

5.3 SOLAR CELL FABRICATION AND RESULTS $\ldots \ldots \ldots . \ldots \ldots$

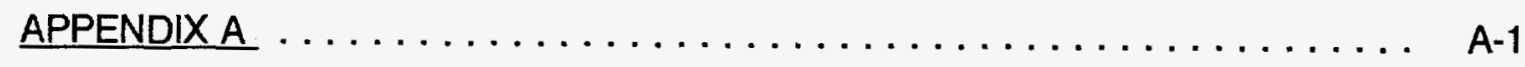

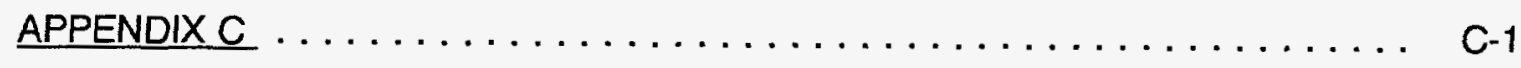




\section{Glossary of Mathematical Terms}

In Section 2

$\eta$

$V_{\text {oc }}$

$R$

$R_{\mathrm{T}, 0}$

$A_{T, 0}$

calf, cbet, cgam

$\mathrm{xO}, \mathrm{yO}, \mathrm{zO}$

$x 1, y 1, z 1$

$a, b, c$ calfn, cbetn, cgamn

$\theta$<smiles>C1C[Pb][Pb]1</smiles>

L

N

$\|\cdot\|$

$\otimes$

$\odot$

In Section 3

f

v

$\nabla$

$\nabla_{k}$

$\mathbf{F}$

$(\partial f / \partial t)_{c}$

$\mathrm{R}$

$R_{\text {th }}$

$P$

G

$m_{c e}$

$\tau_{2}$

$\tau_{3}$

$\tau_{4}$ *

$\mathrm{m}$

$W$

$W_{0}$

$\boldsymbol{\epsilon}$

$<.>$

$m_{i}$

$T_{e, h}$

$\eta_{c, v}$

$F_{j}($.
Index of refraction of material

Open Circuit Voltage

Reflectance of a surface

Reflectance of a textured cell or planar cell, respectively

Area of a textured cell or planar cell, respectively

Direction cosines of a ray, relative to cartesian coordinates

Triplet describing point of origin of a ray

Triplet describing point of impact of a ray

Intercepts of the coordinate axes and mathematical plane

Direction cosines of the normal of a surface

Angles of incidence and reflectance, respectively

Vector pointing from point $P_{0}$ to $P_{1}$

Vector representation of the direction cosines of a line

Vector representation of the direction cosines of a surface normal

Magnitude of a vector

Symbol representing the vector cross product

Symbol representing the vector dot product

Distribution function

Velocity vector

Gradient operator in real space

Gradient operator in $\mathbf{k}$ (momentum) space

External force

Change in distribution due to collisions

Recombination events (SRH, Auger, Band-to-Band)

Thermal resistance of a system

Power

Generation events

Conductivity effective mass

Momentum relaxation time

Energy relaxation time

Energy flux relaxation time

Density of states effective mass

Kinetic energy

Kinetic energy in equilibrium

Potential energy

Average value of parameter

Effective mass in basis direction $\mathbf{i}$

Electron and hole distribution temperatures

Reduced energy of conduction and valance bands

Normalized Fermi integral of order $\mathrm{j}$ at reduced energy 


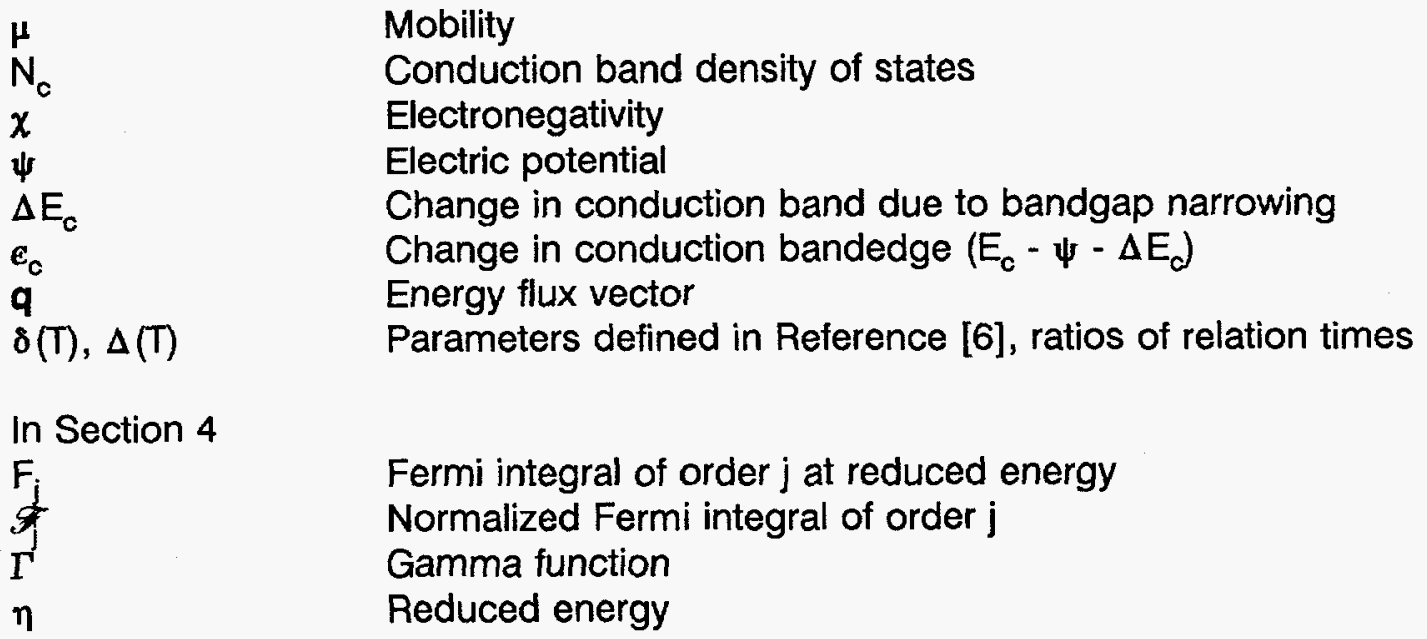




\section{SUMMARY}

This annual report covers the research conducted on modelling and development of high-efficiency silicon solar cells during the period May 1989-August 1990. First, considerable effort was devoted toward developing a ray tracing program for the photovoltaic community to quantify and optimize surface texturing for solar cells. Second, attempts were made to develop a hydrodynamic model for device simulation. Such a model is somewhat slower than drift-diffusion type models like PC-1D, but it can account for more physical phenomena in the device such as hot carrier effects, temperature gradients, thermal diffusion and lattice heat flow. In addition, Fermi-Dirac statistics have been used in the model to deal with heavy doping effects more accurately. Third and final component of the research includes development of silicon cell fabrication capabilities and fabrication of high-efficiency silicon cells.

The technical progress in this report is divided into four chapters. Each chapter is self contained including the figures and references. First chapter (Section 2) describes the development of the ray tracing model (TEXTURE). This program is now complete and is being used for modelling and optimization of light trapping characteristics of solar cells as a result of front and back surface texturing. The model gives the true carrier generation profile and also provides quantitative information about the percent of rays absorbed at each wavelength, number of photons lost from the sides of the cell, photon loss from the back due to imperfect reflector, and increase in surface area due to texture. The model calculates the maximum short-circuit current, assuming $100 \%$ collection, to 
indicate the light trapping efficiency of the texturing scheme being analyzed. Preliminary attempts have been made to analyze various promising surface texturing schemes for silicon cells. In addition, some initial calculations have been done to investigate the merit of texturing GaAs, CdTe and CulnSe ${ }_{2}$ cells. More work is in progress to demonstrate the value and applicability of this ray tracing program.

Section 3 discusses the development of the hydrodynamic model and its pros and cons compared to drift-diffusion type models and Monte Carlo device modelling techniques. So far, our hydrodynamic model has been developed to a point that it can calculate the equilibrium characteristics of a device. All the equations have been formulated and most of the computer code has been written. Work is now in progress to complete the model so it can predict the transient behavior and can be used for modelling devices like solar cells.

Section 4 deals with the re-evaluation of the Fermi integrals including the derivatives of the half-order Fermi integrals. This was necessary since the hydrodynamic model is based on Fermi Dirac statistics. The Fermi integrals of half orders are important in the simulation of semiconductor transport processes while the first two derivatives are needed for the interpolation. We used numerical integration to calculate and tabulate Fermi integrals ranging from $-1 / 2$ to $5 / 2$ over the reduced energy $(E f-E c / k T)$ range of -5 to 20 in 0.25 increments. It is also shown that values of $F_{-3 / 2}$ or the second derivative of $F_{1 / 2}$ in the literature are incorrect.

Section 5 covers the progress made in the area of process development and 
fabrication of high-efficiency silicon cells. This section describes the effort made toward establishing and improving solar cell processes such as oxidation, phosphorous and boron diffusion, aluminum heat treatment, and two-layer antireflection coating to attain high-efficiency solar cells. Cell fabrication is in progress using $F Z, M C Z$ and $C Z$ silicon. So far, uncoated cells with efficiencies of $\sim 13 \%$ and coated cells with efficienies of $\sim 18 \%$ have been fabricated. 


\section{MODELLING OF LIGHT TRAPPING IN SOLAR CELLS BY TEXTURED SURFACES}

2.1 History of the Analysis of Textured Solar Cells

Conventional single crystal solar cells consist of planar front and back surfaces. This is a result of the single crystal being wafered from a boule of the material and subsequently being polished to remove surface damage created during the wafering process. High-efficiency cells created on these wafers have short-circuit currents approaching the amount of electron-hole pairs created by the absorbed light. However, the quantity of light which is absorbed is a small fraction of the amount which is incident upon the cell. A portion of the light cannot be absorbed due to the energy of the photon being less than the bandgap of the material; this is a non-recoverable loss. Some of the light is reflected from the front surface of the cell. This loss may be reduced by an appropriately designed anti-reflection coating consisting of thin layers of dielectric material with various indices of refraction and thicknesses. Another loss of photons involves those which escape though the back of the cell, usually those with small absorption coefficients. One way to minimize this loss is by the application of a back surface reflector created by a quarter wavelength dielectric material coated with a highly reflective metal. The back loss may also be reduced by an increase in the thickness of the cell; unfortunately, this is costly and leads to a reduction in the open-circuit voltage of the cell. Even with these advances, a significant portion of the useable photons are not utilized. Most of these photons are in the long wavelength regime where the absorption coefficient is small. Therefore, most of the photons which are reflected from the back surface reflector are coupled out the front of the cell after making two passes through the 
material. The way to 'trap' the light within the cell material is to have the photons totally internally reflected at the semiconductor/air interface. This implies that either the angle of incidence of the light reflected from the back must be other than normal or the front surface must no longer be planar. To change the angle of incidence of the light coming from the back to the front of the cell requires that the back surface of the cell be textured. Thus, it follows that to further increase the short-circuit current of a solar cell requires at least one surface of the cell be textured.

The first textured cell was created in 1960 by Dale and Rudenberg [1]. Their surface texturing, shown schematically in figure 2-1, was inverted tetrahedrons (the authors called them inverted pyramids, we have changed the name) created by ultrasonically cutting the surface of the cell. Their goal, in which they succeeded, was to

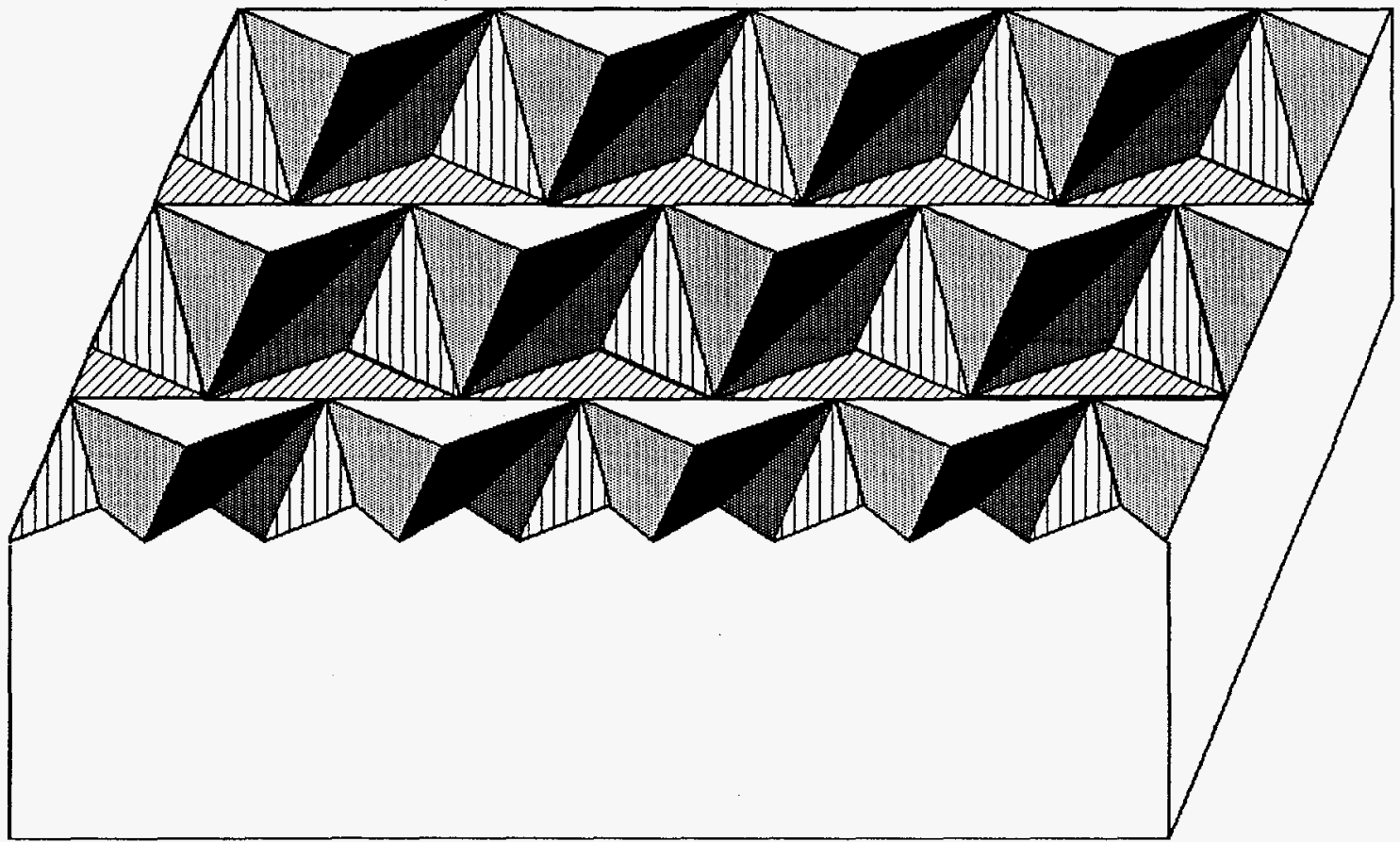

Figure 2-1. Schematic of inverted tetrahedrons created by ultrasonically cutting the surface of silicon, created in 1960 by Dale and Rudenberg. 
reduce the front-surface reflectance of bare silicon from $\sim 30 \%$ to $4 \%$. The final efficiencies of their cells were "comparable to the best of the plain coated cells." This was in spite of the fact that the surface texture increases the series resistance of the cell and, without proper oxide passivation, could lead to a reduction in open-circuit voltage.

In 1969, A.E. St. John [2] patented a method of creating multiple internal reflections for fast optical detectors. The surface of the silicon was roughened by sandblasting. This leads to random surface structures of various angles, figure 2-2. Since the light is refracted into the material at all angles, the average path length through the material is increased. Also, any light returning from the back surface would impinge upon a random surface and a significant fraction ( $-92 \%)$ would be totally internally reflected leading to further photon collection. A surface with random surface structures is also called Lambertian.

The Lambertian surfaces have not, as of yet, produced high-efficiency singlecrystal solar cells. This may be a result of the surface damage left by the texturing process. On the other hand, the Lambertian design is applied with some success to

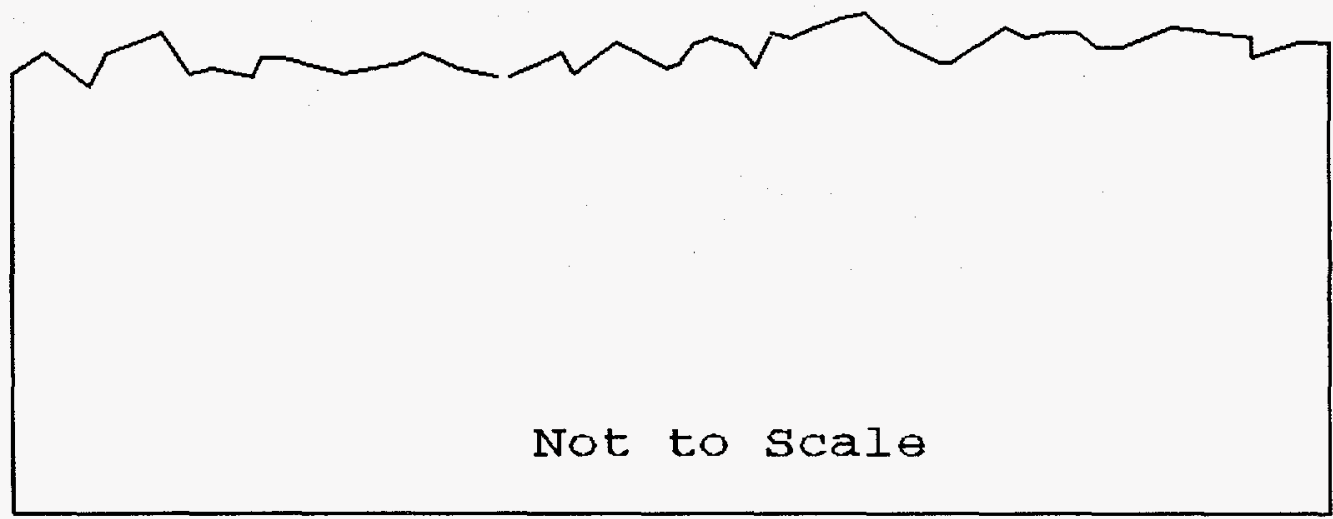

Figure 2-2. Cross section of a Lambertian surface, not drawn to scale. 
polycrystalline solar cells. The surface roughness is created by the grains of the material having different heights and orientations. Some of the material systems which use Lambertian surface texturing include $\mathrm{CdS}-\mathrm{Cu}_{2} \mathrm{~S}$ [3], $\alpha-\mathrm{Si} \mathrm{[4],} \mathrm{and} \mathrm{CulnSe}{ }_{2}$ [5]. The mathematical analysis of the Lambertian structure has been studied by several researchers $[6,7,8]$ using various techniques. The conclusion drawn from the analysis is that the design produces a maximum path length enhancement of $\sim 4 \eta^{2}$, where $\eta$ is the index of refraction of the material. This path length enhancement leads to greater spectral response in the red region of the solar spectrum.

It was not until 1974 that significant work on geometrically textured solar cells was again reported. In that year both Arndt [9] and Baraona [10] presented silicon cells with surfaces covered in pyramids. These surface structures were created by anisotropically etching the front surface of $(100)$ silicon. The etches (buffered $\mathrm{KOH}$ or Hydrazine hydrate) preferentially expose the (111) planes which form the pyramids. If the unprotected wafer were placed directly in the etch, pyramids of random height would form. This leads to an improvement in the short-circuit current of approximately $20 \%$. However, if the surface was oxidized and then line patterns opened in the oxide prior to the etch, uniform pyramids or v-grooves, figures 2-3a and 2-3b, could be created. Again,

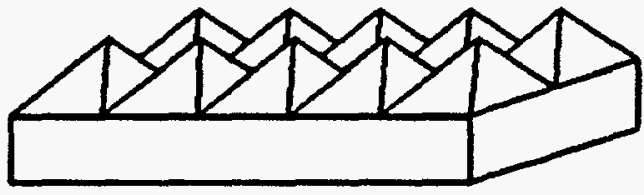

A

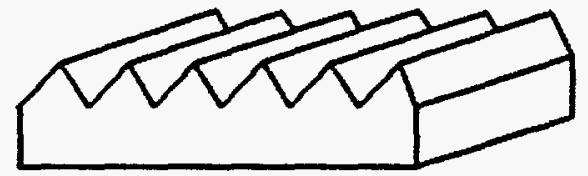

$\mathbf{B}$

Figure 2-3. Surface textures created by the first anisotropic etch: A.) pyramids, B.) v-grooves. 
an improvement in the cell performance was observed.

In 1984, anisotropically etched GaAs solar cells were introduced by Roedel and Holm [11]. Structures with v-grooves and inverted v-grooves were analyzed based upon the reduction of front surface reflectance; the increase in path length of the light was not taken into account. However, analytical formulas for estimating the changes in cell output parameters with various surface texturing designs were presented. The most important of these is the change in open-circuit voltage, which actually decreases as the surface area of the cell is increased. This decrease in voltage is offset by a larger increase in the short-circuit current thus leading to higher cell efficiencies. The expression for the change in voltage is given as, assuming the dominant recombination mechanism is area dependent,

$$
\Delta V_{o c}=\frac{K T}{q} \ln \left[\frac{\frac{\left(1-R_{T}\right)}{\left(1-R_{\partial}\right)}}{\frac{A_{T}}{A_{0}}}\right]
$$

Where $A_{T}$ and $R_{T}$ are the surface area and reflectance of the textured cell, $A_{0}$ and $R_{0}$ are the surface area and reflectance of the planar cell. This equation will usually over estimate the loss in open-circuit voltage because it does not take into account the enhancement in current due to the path length increase. Still, the authors predicted increases in GaAs cell efficiencies of $51 \%$ and $38 \%$ for the v-groove and inverted v-groove structures respectively, inverted v-grooves are in the shape of triangles with small openings in the top surface of the material.

The mathematical analysis for the path length enhancement of geometrical 


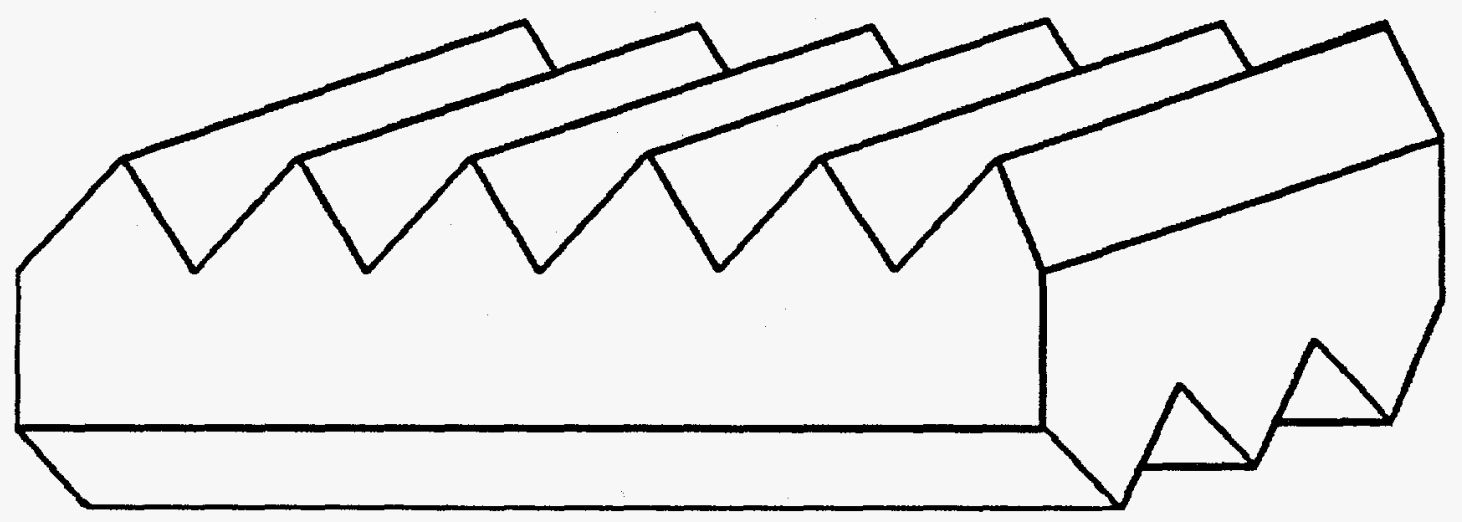

Figure 2-4. Example of perpendicular slats geometry proposed by Landis.

texturing was undertaken by Green and Campbell in 1987 [12]. Using a ray tracing program, the number of passes a ray makes through a cell was traced until the ray was coupled out of the cell. The surface structures studied included v-grooves, Lambertian, pyramids, brickwork pyramids, and some double sided geometries, such as pyramids on both sides and perpendicular slats (proposed by Landis), figure 2-4. The authors concluded that due to path length enhancement and light trapping properties, the perpendicular slats geometry could out perform the Lambertian design. However, the authors did not take into account surface reflectance losses.

In 1988, these same authors introduced the tilted pyramid design [13], in which the faces of the pyramid were purposefully made non-symmetrical. The design approached the characteristics of the Lambertian design, and a simple manufacturing technique was proposed. In addition, it was proposed that with the addition of a flat cover glass, the path length enhancement could be further increased. This was due to rays being internally reflected from the air/glass interface due to the angular dependence of the light after striking the tilted surfaces of the pyramids.

Also in 1988, Uematsu [14] introduced the parallel slat geometry for ultra thin 


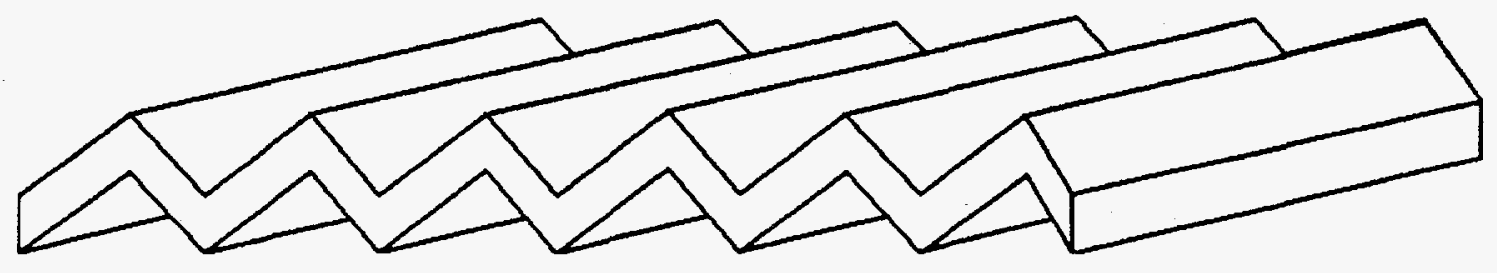

Figure 2-5. Example of the parallel slats geometry proposed by Uematsu.

devices. The device consisted of parallel v-grooves on both the front and back surface of the cell, figure 2-5. The predicted current was smaller than other texturing designs, but larger than that of a flat cell. The smaller current was offset by making the cells very thin to increase the open-circuit voltage.

Experimental verification of light trapping in perpendicular and parallel slats geometries was reported in 1988 by Keavney et al [15]. The amount of light trapping was found by placing the samples in an integrating sphere, the total reflection and transmission were measured and combined to calculate the amount of absorption. As predicted from the previous theoretical work, the perpendicular slats produced the highest absorption. However, when solar cells were fabricated on the samples, the perpendicular slat geometry performed poorly due to the back metal contact being applied directly to the silicon. This reduces the effectiveness of the back-surface reflectance and acts as a recombination sink for carriers.

Cell modelling has lagged behind both the experimental verification of light trapping effects in cells and the modelling of the current enhancement due to light trapping. Part of this may be due to the difficulty in accurately describing the photon generation profile in the geometric structures. The earliest attempt to model cells by Basore with light trapping used the Lambertian design [16]. Since the front surface texture was small compared to the cell thickness, the surface could be modelled as a 
conventional cell with flat surfaces and a high surface recombination velocity at the front surface. The generation profile was created by increasing the photon flux and angle of incidence of incoming light until the amount of photons absorbed matched an analytical model. Efficiencies in excess of $24 \%$ were predicted with fairly high surface recombination velocities. Landis [17] was the first to attempt to model the perpendicular slats geometry. The approach to the generation profile was similar to that just described. Unfortunately, it is unclear if the increase in surface area was considered in the calculations. The front and back surface recombination velocities were modelled as 10 $\mathrm{cm} / \mathrm{s}$. Also, the texture heights to cell thickness were not reported, making it unclear if the author took into account generation profile effects. Other types of cells have been modelled with the assumption that the surface texture dimensions are much less than the thickness of the cell. It was shown that the relative enhancement in cell efficiency due to light trapping is much greater for cells with lower lifetimes. This is a result of the carriers being generated closer to the collecting junctions and the fact that for high lifetime material thick base cells most of the photogenerated electron-hole pairs were collected in the first place.

The next step in textured cell modelling was taken in 1988 with the introduction of PC-1D version 2 [18], a cell modelling program. The program uses a quasi-one dimensional model to describe the textured cell. By letting the area be a function of depth into the device, the increase in surface area of both the front and back surface texture is incorporated into the cell simulation. In addition, the program accepts generation profiles created by any method, allowing the incorporation of two dimensional effects of the surface texture.

In 1989, both the UNSW [19] and Stanford University [20] independently 


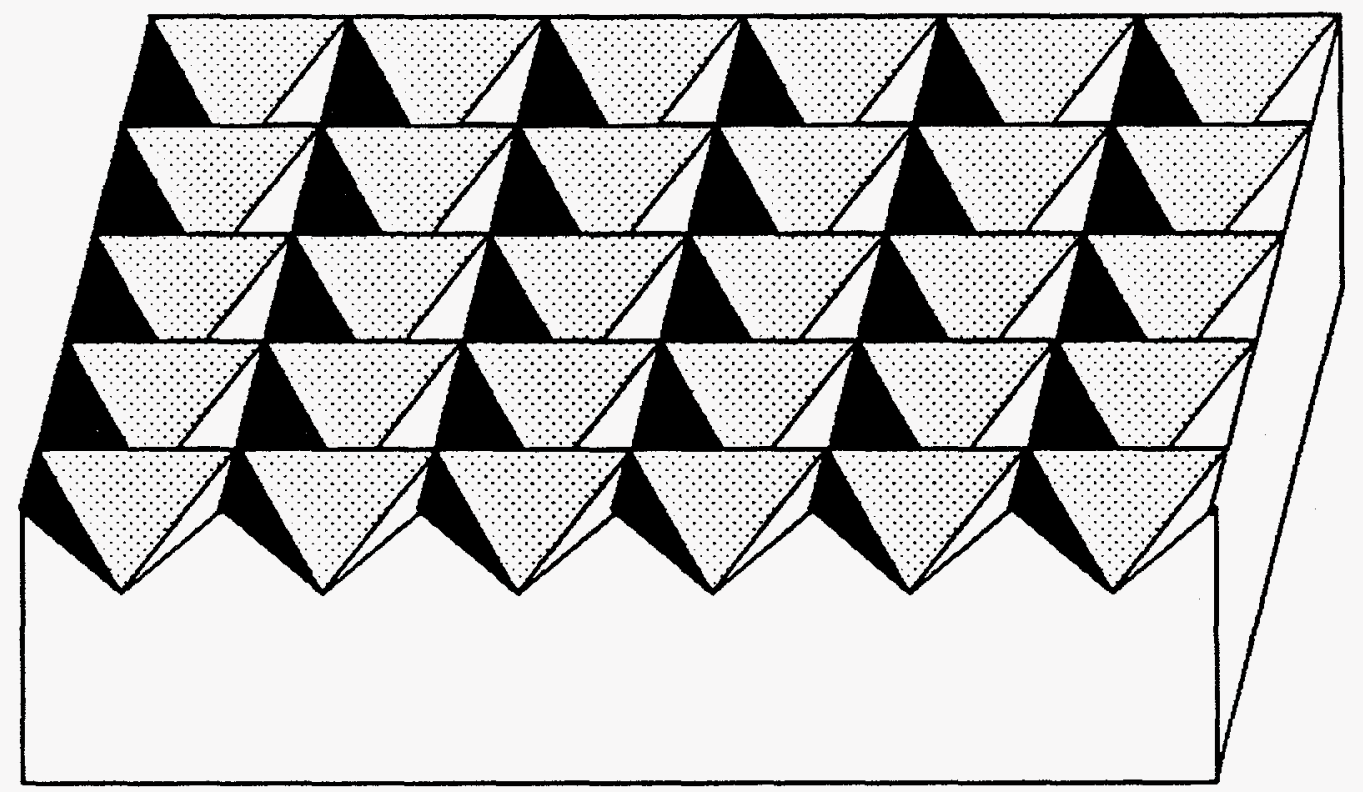

Figure 2-6. Example of inverted pyramids, the last row of pyramids has been cut away to show geometry.

introduced cells with inverted pyramids on the front surface, figure 2-6. It was reported that cells with this surface structure consistently produced higher short-circuit current densities than the normal pyramid structure. Currently, a theoretical analysis of this structure has not been reported.

Although textured cells have been produced for 30 years, the technology is still growing. Different anisotropic etches are being explored; in addition better lithography techniques are being developed. These advances will lead to new surface textures and finer structures. In addition, laser patterning and ultrasonic cutting should still be explored, even if these are currently not economically feasible for mass production. 


\subsection{Development of Ray Tracing Model: TEXTURE}

\section{Mathematics of Analysis}

Ray tracing concepts have been used extensively in the analysis of geometric optics. In the area of solar energy, ray tracing has been used to design solar concentrator lenses and solar thermal systems. In terms of solar cell applications, ray tracing is a relatively 'new' technique, but the equations are the same as for the other applications.

\section{Intersection of Lines and Planes}

The mathematics of ray tracing is based on the geometric concepts of the intersection of lines and planes. In addition, Snell's law of refraction and the law of reflection must be accommodated to provide the direction of the lines (rays) after striking a surface. Complexity arises when the possibility of an intersection with a mathematical plane is possible but a physically realistic intersection would not be obtained.

The parametric equation of a line is given by

$$
\frac{x 1-x 0}{c a l f}=\frac{y 1-y 0}{c b e t}=\frac{z 1-z 0}{c g a m}
$$

where $x 1, y 1$, and $z 1$ are the coordinates of the point of intersection, $x 0, y 0$, and $z 0$ are the original point of origin. The direction cosines of the line (calf, cbet, cgam) are found from either Snell's law or the law of reflection. The equation of a general plane, shown in figure $2-7$ is given by 


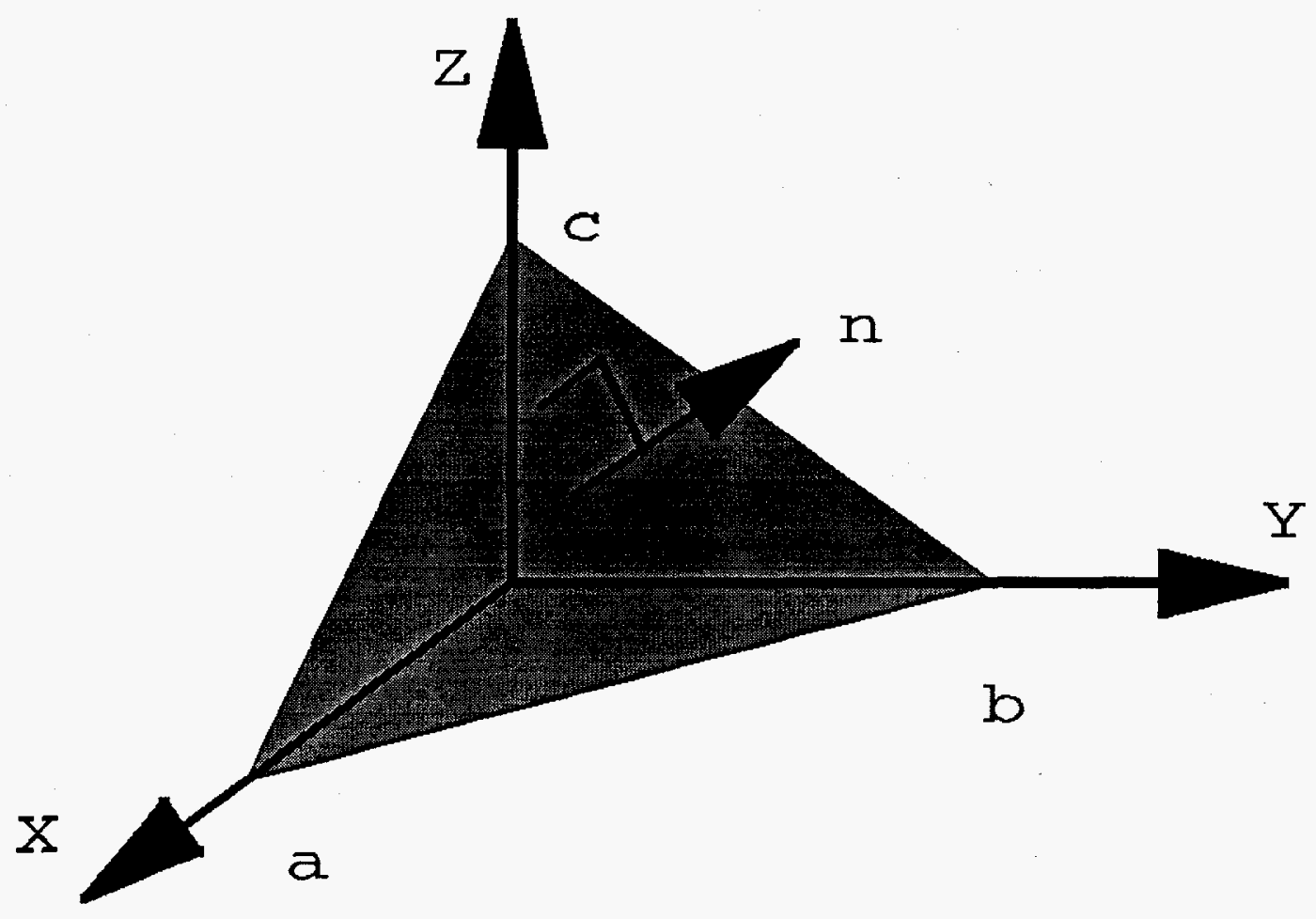

Figure 2-7. A general plane showing the intercepts of the plane and axes.

$$
\frac{x 1}{a}+\frac{y 1}{b}+\frac{z 1}{c}=d
$$

where $a, b$, and $c$ are the intercepts of the $x, y$, and $z$ axes respectively. The direction cosines of the normal of the plane, relative to the cartesian coordinates, are given as follows:

$$
\begin{aligned}
& \text { calfn }=\frac{a}{\sqrt{a^{2}+b^{2}+c^{2}}} \\
& \text { cbetn }=\frac{b}{\sqrt{a^{2}+b^{2}+c^{2}}} \\
& \text { cgamn }=\frac{c}{\sqrt{a^{2}+b^{2}+c^{2}}}
\end{aligned}
$$


The calculation of these angles will become important for the angles of refraction and reflection. The point of intersection is found by solving equations (2-1) and (2-2) simultaneously for one of the three unknown coordinates. For example, if given $x 0$, yo, $z 0$, calf, cbet, cgam, calfn, cbetn, and cgamn, the $z$ coordinate of the point of intersection between the line and plane is

$$
z 1=\frac{d \text { cgam }- \text { calfn(xOcgam }-z 0 \text { cal })- \text { cbetn }(y 0 \text { cgam }-z 0 c b e t)(2-7)}{\text { calfcafin }+ \text { cbetcbetn }+ \text { cgamcgamn }}
$$

This formula will provide valid mathematical coordinates provided the line is not parallel to the plane; i.e. the denominator is non-zero. If calf and cbet are non-zero, then $x 1$ and y1 may be calculated from equation (2-1). If either angle of the above direction cosines is zero, then that coordinate position is the same as the initial point; i.e. $x 1=x 0$ or $y 1$ $=$ y0. Mathematically, this new point of intersection $(x 1, y 1, z 1)$ is contained on the mathematical plane but there is the possibility that it is not contained on the physical plane, see figure 2-8. Therefore, the point of intersection is checked to be sure that it is on the physical surface being considered.

\section{Refraction of Light}

Once it is determined that the ray hits the surface, the direction cosines of the reflected or refracted ray must be determined to follow the ray any further. Figure 2-9 shows an example of Snell's law of refraction; the reflected ray is also included. The plane of the paper is called the refraction (or reflection) plane, and the normal of the plane is perpendicular to the incoming ray and surface normal. Since the incoming ray and the surface normal direction cosines are known, the cross product of these two 


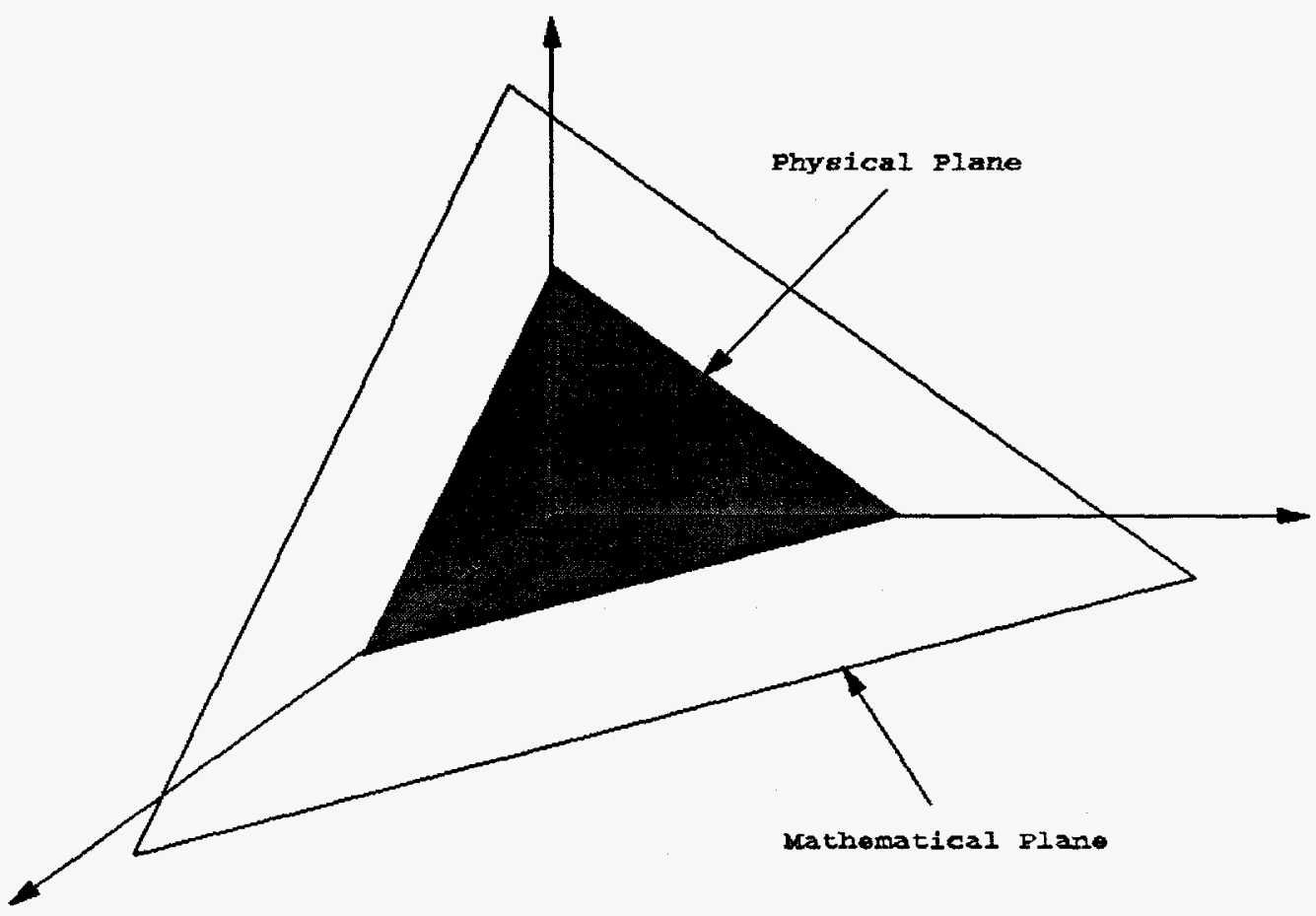

Figure 2-8. Concept of mathematical plane intersection and physical plane intersection.

vectors is the refraction plane normal.

$$
\text { refraction plane normal }=\text { incident ray } \otimes \text { surface normal }
$$

Carrying out the vector product leads to the following equations for the direction cosines of the refraction plane normal:

$$
\begin{aligned}
& \text { calfp = cbet } \times \text { cgamn }- \text { cbetn } \times \text { cgam } \\
& \text { cbetp }=\text { calfn } \times \text { cgam }- \text { cgamn } \times \text { calf }
\end{aligned}
$$




$$
\text { cgamp }=\text { calf } \times \text { cbetn }- \text { calfn } \times c b e t
$$

Therefore, since the refracted ray is also perpendicular to the refraction plane normal, the dot product of the two vectors will be zero. Mathematically this is equivalent to

$$
0=\text { calfr } \times \text { calfp }+ \text { cbetr } \times \text { cbetp }+ \text { cgamr } \times \text { cgamp }
$$

Where calfr, cbetr, and cgamr are the unknown direction cosines of the refracted ray.

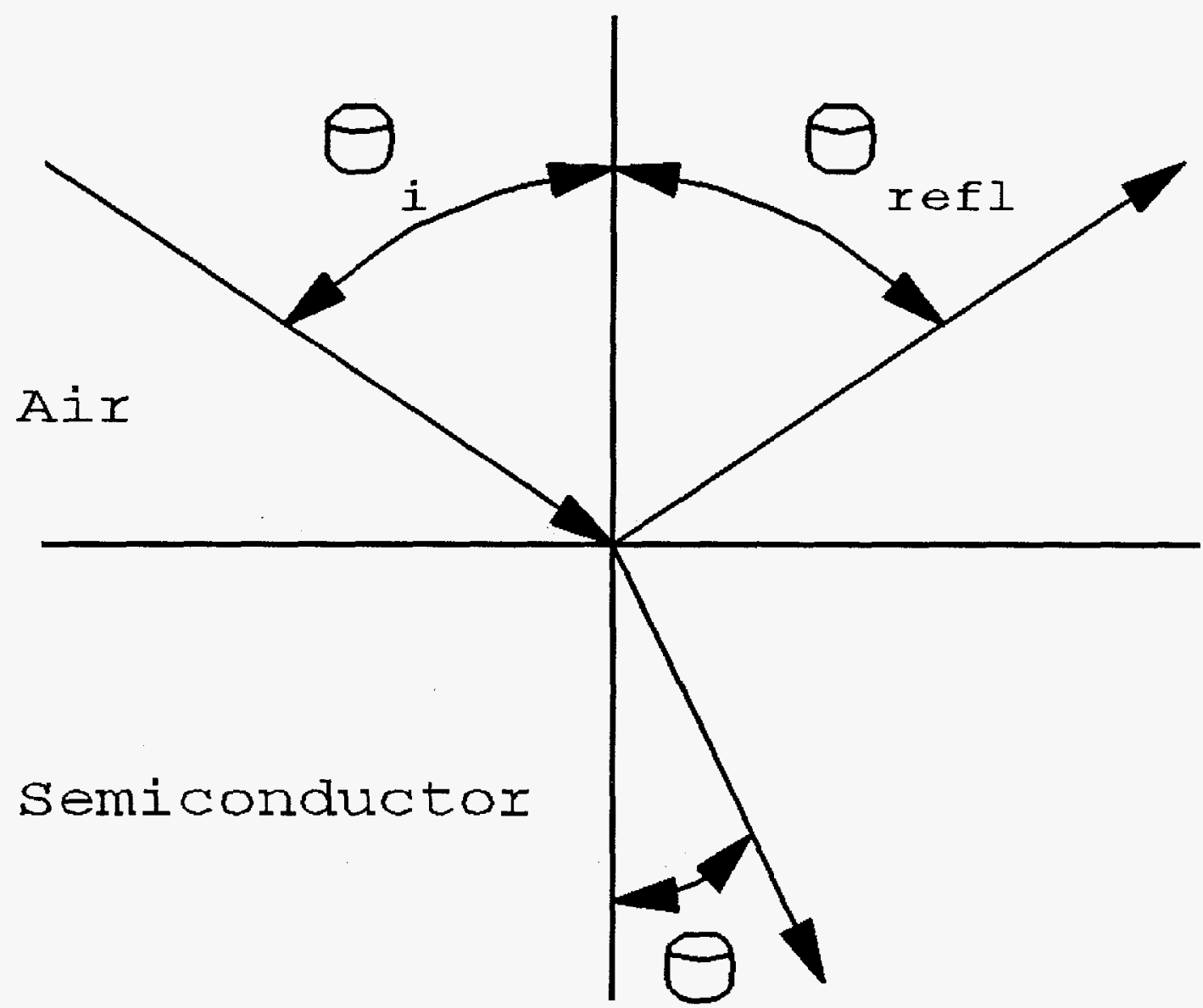

refr

Figure 2-9. Example of Snell's law of refraction, the reflected ray is also present. 
The next condition on the refracted ray is given by Snell's law as follows: First, the angle between the surface and the incoming ray is found from the dot product of the surface normal and the incoming ray

$$
\cos \left(\theta_{i}\right)=\text { calf } \times \text { calfn }+ \text { cbet } \times \text { cbetn }+\operatorname{cgam} \times \operatorname{cgamn}
$$

Then, by a re-arrangement of Snell's law from

$$
\frac{\sin \left(\theta_{i}\right)}{\sin \left(\theta_{r}\right)}=\frac{\eta_{r}}{\eta_{i}}
$$

to the following form, to solve for $\theta_{r}$,

$$
\cos \left(\theta_{r}\right)=\cos \left(\arcsin \left(\sin \left(\theta_{i}\right) \frac{\eta_{r}}{\eta_{i}}\right)\right)
$$

This relates the incoming angle to the cosine of the refracted angle. This is equivalent to the dot product of the surface normal and the refracted ray

$$
\cos \left(\theta_{r}\right)=\text { calfr } \times \text { calfn }+ \text { cbetr } \times \text { cbetn }+ \text { cgamr } \times \text { cgamn }
$$

The third and final condition will be given by the angle between the refracted ray and the incoming ray. This is given by the sum of the two angles

$$
\cos \left(\theta_{r i}\right)=\cos \left(\arccos \left(\theta_{i}\right)+\arccos \left(\theta_{r}\right)\right)
$$

which is equal to the dot product between the incoming ray and the refracted ray 


$$
\cos \left(\theta_{r}\right)=\text { calfr } \times \text { calf }+ \text { cbetr } \times \text { cbet }+ \text { cgamr } \times c g a m
$$

This leads to a three-by-three system of equations for the unknowns calfr, cbetr, and cgamr, given in vector-matrix form by

$$
\left[\begin{array}{ccc}
\text { calfp } & \text { cbotp } & \text { cgamp } \\
\text { calfn } & \text { cbetn } & \text { cgamn } \\
\text { calf } & \text { cbet } & \text { cgam }
\end{array}\right]\left[\begin{array}{c}
\text { calfr } \\
\text { cbetr } \\
\text { cgamr }
\end{array}\right]=\left[\begin{array}{c}
0.0 \\
\cos \left(\theta_{j}\right) \\
\cos \left(\theta_{n}\right)
\end{array}\right]
$$

This system is solved by the method of determinants for the new direction cosines of the refracted ray, calfr, cbetr, and cgamr. These values are then substituted for the old values of the direction cosines (calf, cbet, cgam) and give the new direction the ray is travelling.

\section{Reflection of Light}

For the angle of reflection, the first condition is the same as for a refracted ray; i.e. the ray must be perpendicular to the normal of the plane of reflection. The second condition to determine the reflected ray is that the angle of incidence is equal to the angle of reflection relative to the surface normal,

$$
\cos \left(\theta_{r}\right)=\cos \left(\theta_{i}\right)
$$

Therefore, the following relationship holds, for the dot product of the reflected ray and the surface normal,

$$
\cos \left(\theta_{i}\right)=\text { calfr } \times \text { calfn }+ \text { cbetr } \times \text { cbetn }+ \text { cgamr } \times \text { cgamn }
$$


The third and final condition on the reflected ray, which also comes from the fact that the angle of incidence equals the angle of reflection, is the angle between the incident ray and the reflected ray is $2 \theta_{i}$. The following equation is created by taking the dot product of the reflected ray with the incoming ray,

$$
\cos \left(2 \theta_{i}\right)=\text { calfr } \times \text { calf }+ \text { cbetr } \times \text { cbet }+ \text { cgamr } \times \text { cgam }
$$

Arranging these three conditions into a linear set produces

$$
\left[\begin{array}{ccc}
\text { calfp } & \text { cbetp } & \text { cgamp } \\
\text { calfn } & \text { cbetn } & \text { cgamn } \\
\text { calf } & \text { cbet } & \text { cgam }
\end{array}\right]\left[\begin{array}{c}
\text { calfr } \\
\text { cbetr } \\
\text { cgamr }
\end{array}\right]=\left[\begin{array}{c}
0.0 \\
\cos \left(\theta_{i}\right) \\
\cos \left(2 \theta_{i}\right)
\end{array}\right]
$$

Again this system is solved by the method of determinants to produce the new direction cosines of the reflected ray. In addition, if the surface being considered is the top of the cell or the top of the cover glass and the direction of travel is outward, then the angle of incidence is checked against the critical angle. The critical angle is given by

$$
\sin \left(\theta_{c}\right)=\frac{\eta_{r}}{\eta_{i}} \text { with } \eta_{i}>\eta_{r}
$$

If the angle of incidence (equation (2-21)) is smaller than the critical angle then ray is coupled out of the material and the new angle of refraction is calculated. 


\section{"Constrained" Volume}

The direction of reflected and refracted rays and the intersection of rays and surfaces may be calculated using the above analysis. An example of a ray bouncing within a textured cell is shown in figure $2-10$. However, the physical surfaces must be

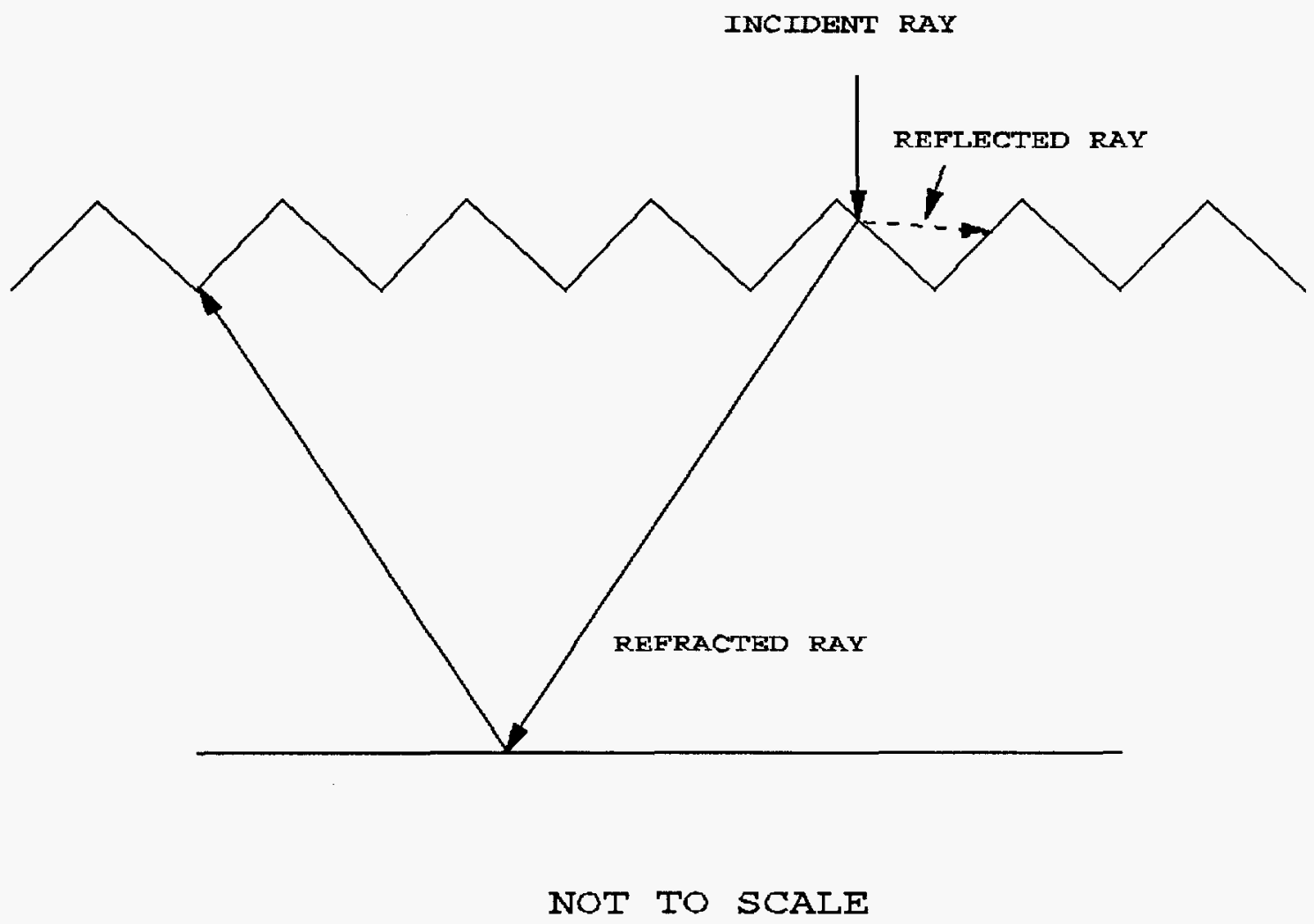

Figure 2-10. Example of path of ray through a textured cell, the reflected ray is shown. modelled mathematically within the computer. One option is to use a Fourier series to describe the textured surface $(z=F(x, y))$. Unfortunately, the general Fourier series for pyramids and tetrahedrons would be quite complex. The alternative is to use the symmetry of the textured surface to some advantage. The following description applies to finding the intersection of a ray and a pyramidally textured surface; however, a similar analysis also applies for other surfaces. First, assume the ray has already entered the 


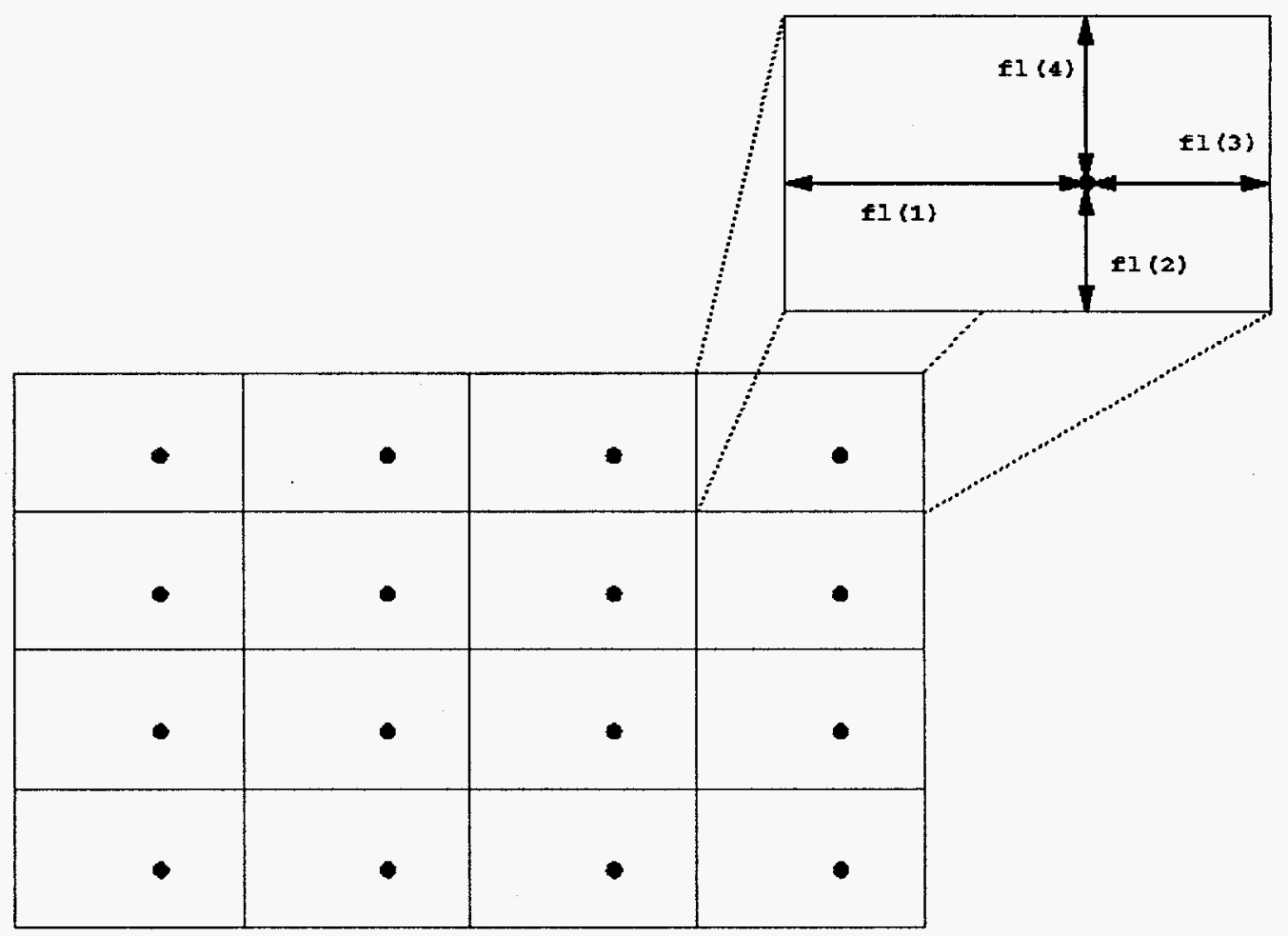

Figure 2-11. Pattern of rectangles on the front surface of a textured cell.

cell and has been reflected up from the back surface reflector. If the plane located at the substrate thickness is imagined from the ray's perspective, it appears to be a series of regular repeating rectangles, figure $2-11$. The $X Y$ dimensions of the rectangle are depicted in the figure $2-11$ and these values are specified by the designer. These dimensions also determine the peak position of the pyramid. The peak of the pyramid is located above the dot at a height specified by the designer. The intersection of the ray and the plane located at the substrate thickness (the bottom of the pyramids) is given by, 


$$
\begin{gathered}
z 1=\text { thickness } \\
x 1=x 0+\left[\frac{(\text { thickness }-z 0)}{\text { cgam }}\right] \text { calt } \\
y 1=y 0+\left[\frac{(\text { thickness }-z 0)}{\text { cgam }}\right] \text { cbet }
\end{gathered}
$$

where $x 0, y 0$, and $z 0$ are the coordinates of the point of origin of the ray on the back surface. The direction cosines calf, cbet, and cgam were calculated using the reflection analysis described in the reflection section. The coordinates $x 1, y 1$, and $z 1$ designate where the ray has struck the plane located at the substrate thickness. The rectangle in which the ray hits the substrate plane is then determined by the knowledge of the characteristic dimensions. The $X Y$ coordinates of the peak of the pyramid are recorded as an offset (xoffset, yoffset). Now define the location of the peak of the pyramid projected onto the base plane as the origin of a local coordinate system. The point of intersection may be expressed in terms of the local coordinate system as 


$$
\begin{aligned}
& z I=z 1-\text { thick } \\
& x I=x 1-x o f f s e t \\
& y I=y 1-\text { yoffset }
\end{aligned}
$$

where $\mathrm{xl}, \mathrm{yl}$, and $\mathrm{zl}$ are the coordinates in the local system. Notice that every pyramid can be mapped into the reference pyramid by a suitable set of offsets. Therefore, routines need only be written to examine individual texturing geometries with appropriate offsets. Figure 2-12 shows an example of a reference pyramid. Similar structures may be created for other geometries, where the point of intersection of a ray and the bottom of the structure is designated by the point A. Now, given that the bottom of the pyramid has been struck, the ray is trapped or constrained within the pyramid's volume. The ray may not exit the volume without encountering an exterior surface. Given that the ray is travelling up, and has hit the bottom of the pyramid, only four planes need be checked to see if the ray intersects them. Mathematically, the ray may hit any or all the planes, but it will only hit one which satisfies all of the requirements of the textured physical surface. Therefore, once it has been determined that a plane has been struck, the other planes which have not been checked need not be examined. The coordinates of the point of intersection in local coordinates is found by solving the following equations 


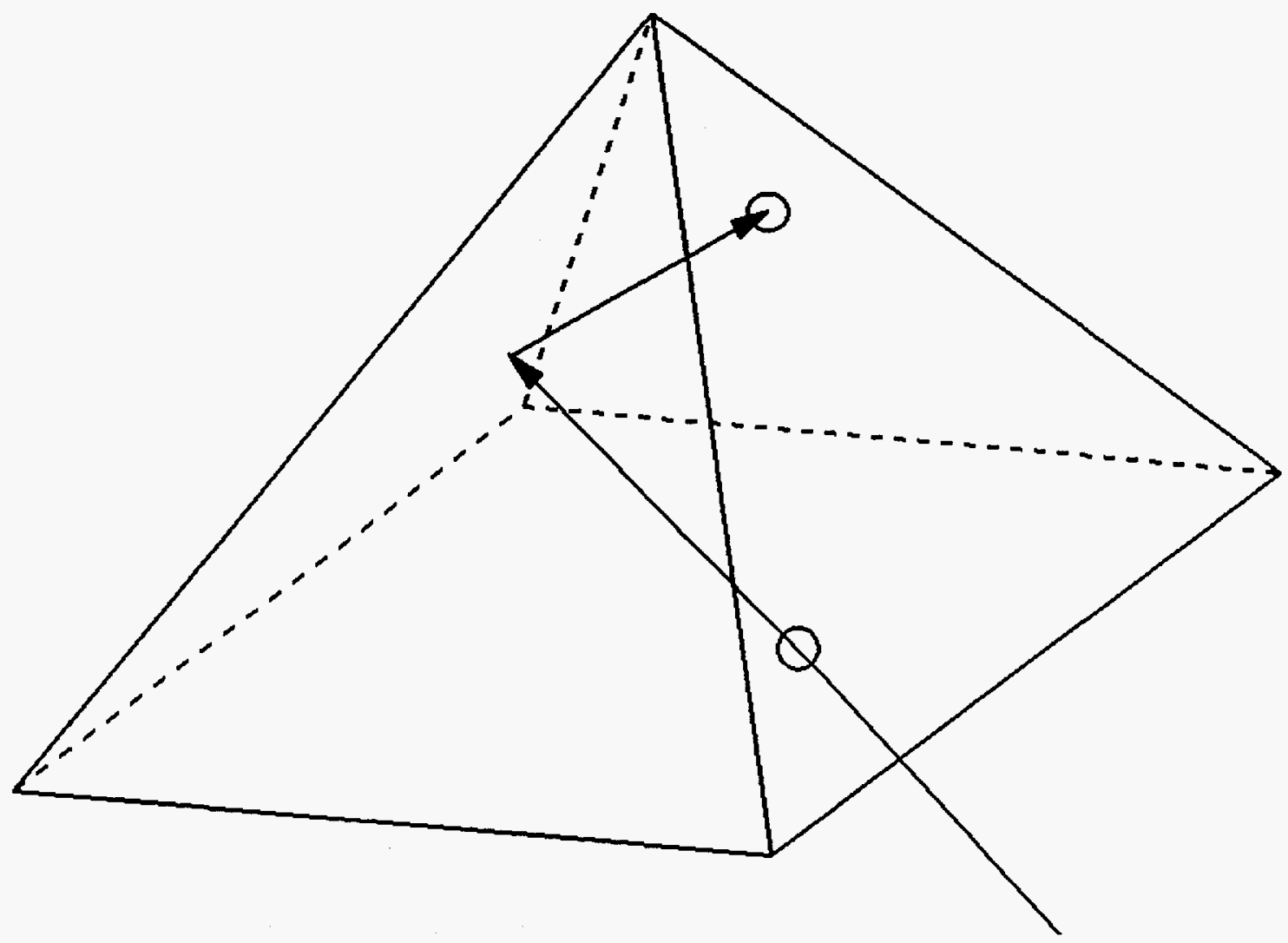

Figure 2-12. Reference pyramid with ray striking bottom and two sides.

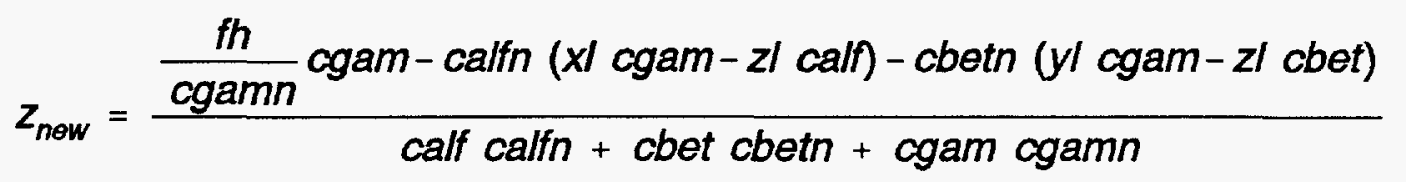

$$
\begin{aligned}
& x_{\text {new }}=x l+\frac{z_{\text {new }} \text { calf }}{\text { cgam }} \\
& y_{\text {now }}=y l+\frac{z_{\text {now }} \text { cbet }}{\text { cgam }}
\end{aligned}
$$


The coordinates $x_{\text {new }}, y_{\text {new }}$, and $z_{\text {new }}$ signify the point of intersection of the ray and one plane of the pyramid, point $B$ in figure 2-12. The direction cosines of the surface normal are found from information provided by the designer in the form of the dimensions of the rectangle; i.e. the intersection of the planes of the pyramid with the coordinate axes. These values of direction cosines of the surface normal take on four different values depending upon which face of the pyramid is being examined. The next step in the analysis is to determine if the ray has struck within the parameters of the physical surface for each plane of the pyramid. Criteria for each coordinate must be met in order for the ray to strike one of the four physical surfaces, and these are

$$
\begin{aligned}
& \text { plane } 1 \\
& \text { plane } 2 \\
& 0 \leq z_{\text {new }} \leq f h \\
& 0 \leq z_{\text {new }} \leq f h \\
& x_{\text {now }}=\frac{z_{\text {new }}-f h}{f h} f(1) \\
& -\frac{f l(1)}{f(2)}\left|y_{\text {new }}\right| \leq x_{\text {now }} \leq \frac{f(3)}{f(2)}\left|y_{\text {new }}\right| \\
& -\frac{f(2)}{f(1)}\left|x_{\text {new }}\right| \leq y_{\text {new }} \leq \frac{f((4)}{f((1)}\left|x_{\text {new }}\right| \\
& y_{\text {new }}=\frac{z_{\text {new }}-f h}{f h} f(2) \\
& \text { plane } 3 \\
& \text { plane } 4 \\
& 0 \leq z_{\text {new }} \leq f h \\
& 0 \leq z_{\text {new }} \leq f h \\
& x_{\text {new }}=\frac{f h-z_{\text {new }}}{f h} f((3) \\
& -\frac{f((1)}{f /(4)}\left|y_{\text {new }}\right| \leq x_{\text {new }} \leq \frac{f(3)}{f(4)}\left|y_{\text {new }}\right| \\
& -\frac{f((2)}{f((3)}\left|x_{\text {new }}\right| \leq y_{\text {new }} \leq \frac{f((4)}{f(3)}\left|x_{\text {new }}\right| \\
& y_{\text {new }}=\frac{f h-z_{\text {now }}}{f h} f(4)
\end{aligned}
$$


The equations on the left are for planes which do not intersect the $y$ axis, while the equations on the right are for planes which do not intersect the $x$ axis. The parameters $f h, f l(1), f(2), f l(3)$, and $f(4)$ are all defined in figure 2-11. If the new coordinates satisfy all the criteria for one plane of the pyramid, then that plane contains the point of intersection. The local coordinates are then converted to global coordinates by the transformation

$$
\begin{aligned}
& z 1=\text { thick }+z_{\text {now }} \\
& x 1=\text { xoffset }+x_{\text {new }} \\
& y 1=\text { yoffset }+y_{\text {new }}
\end{aligned}
$$

After it is determined that the ray strikes a plane of the pyramid, the new angles of the ray are calculated from the reflection analysis, the critical angle criteria is also checked. If the critical angle criteria is violated then the angles of refraction are calculated and the ray escapes. If the critical angle criteria is not violated, the other three sides of the pyramid are checked to see if the ray rehits the front surface. If none of the other surfaces are struck from within and the critical angle has not been violated then the ray is again travelling towards the back. Since, the ray was "trapped" within the reference pyramid until it could no longer strike a textured surface, the term 'constrained volume' was coined to describe how the rays were traced within the textured surface. This same concept of constrained volume may be applied to rays which strike the outer surface of 


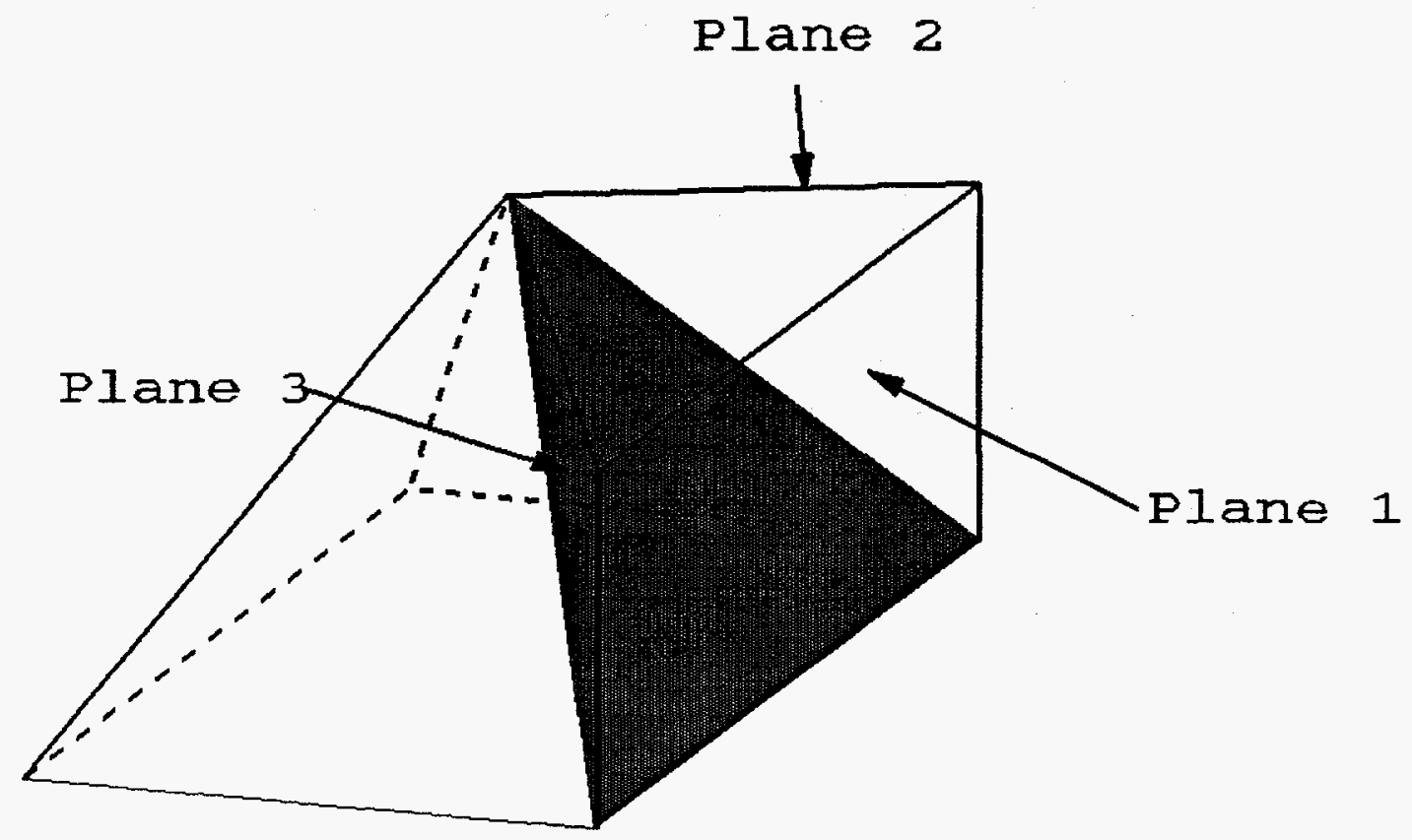

Figure 2-13. Reference pyramid, the real surface is darkened, the imaginary constrained volume is held within planes 1 through 3 and the real surface.

the textured surface as well. In this case, however, different imaginary mathematical planes are used to describe the constrained volume. Figure 2-13 shows an example of the 3 imaginary planes for one side of the reference pyramid. If the ray hits one of the imaginary planes it is moved to the adjoining imaginary volume and tracing continues. This tracing continues until either the ray strikes a real surface or the ray does not hit any of the imaginary surfaces, in which case it has escaped out the top and cannot rehit the surface texture.

\section{Minimum Distance}

Using the above analysis, the path of a packet of photons may be traced as it progresses through the cell structure. The number of photons absorbed will depend 
upon the distance travelled and the absorption coefficient of the material at the wavelength modelled. If the incident flux is $\mathrm{I}_{0}$, the number of photons absorbed is

$$
\text { number absorbed }=I_{0}(\exp (-\alpha(\lambda) 0)-1)
$$

Where $\alpha(\lambda)$ is the absorption coefficient as a function of wavelength, and $d$ is the distance travelled and is given by

$$
d=\sqrt{(x 1-x 0)^{2}+(y 1-y 0)^{2}+(z 1-z 0)^{2}}
$$

The packet of photons is followed until the number of photons falls below a specified level or the ray is coupled out of the cell. Summing all the photons absorbed for all the wavelengths considered produces the maximum short-circuit current possible (assuming $100 \%$ collection of electron-hole paris) with the cell structure. This number may be compared to the number of photons absorbed within a cell structure with flat surfaces to show the enhancement in collection due to light trapping. However, to take into account finite diffusion lengths, generation profiles are required. In the case of a cell with flat surfaces, the generation profile defines how deep within the cell the photons are absorbed. It also reflects the distance from the electrically collecting surfaces or junctions. This concept must be maintained in the transition from the three dimensional profile in a textured cell to the one dimensional generation profile of a flat cell simulation. To accomplish the conversion, the minimum distance from the point of absorption to the nearest collecting surface is calculated, either point A or B in figure 2-14; similar points are also checked on the other three surfaces but are not depicted in this figure. The distance from any point in space to a plane is given by 


$$
D=\frac{\left|N \odot \overline{P_{0} P_{1}}\right|}{|M|}
$$

Where $\mathbf{N}$ is the direction cosines of the surface normal and the vector $P_{0} P_{1}$ is from the point in question to any point on the surface of the plane. The minimum distance will be in a line along the normal of the plane. However, if the point of intersection is not on the textured surface (mathematically correct but physically unrealistic) then the point $B$ is chosen as the collecting surface. In any case, the distance from the point of absorption to the point B is calculated and checked. The formula for the distance from any point,

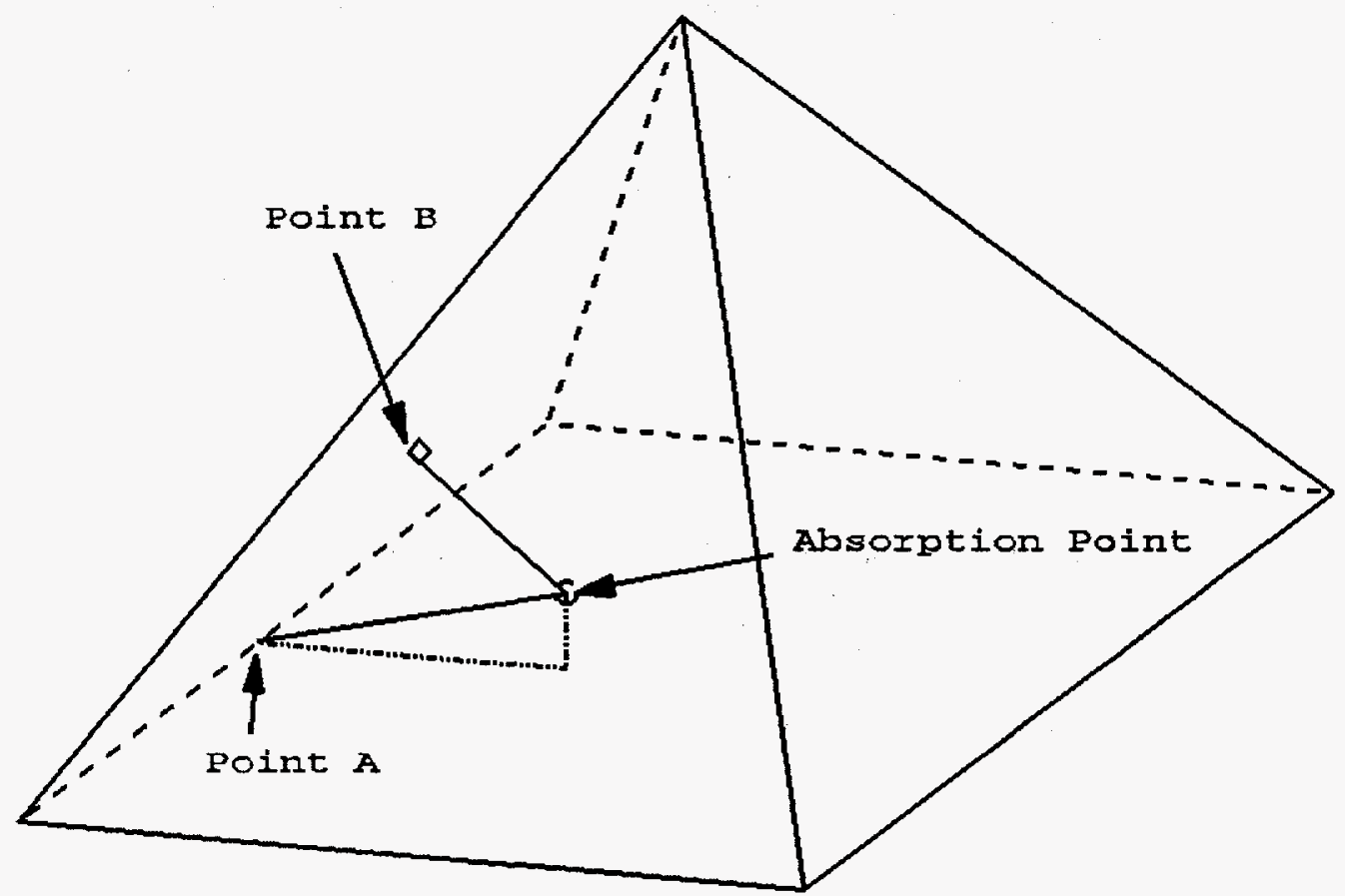

Figure 2-14. Concept of minimum distance from a point of absorption to a collecting surface, minimum distance is from point of absorption to either point $A$ or point $B$. 
not on a line, to a line in space is given by

$$
D=\frac{\left\|L \otimes \overline{P_{0} P_{1}}\right\|}{\|L\|}
$$

where $L$ is the direction cosines of the line, and the vector $P_{0} P_{1}$ is from the point in space to any point that is on the line. A similar procedure finds the distance from the point of absorption to the back surface. The number of photons absorbed at the absorption point are added to the number already assigned at the node located at the distance which corresponds to the minimum of the front or back surface. By following the path of all of the incoming rays and finding the absorption as a function of this effective depth, the one dimensional generation profile for PC-1D (*.GEN files) is produced. The generation profile as a function of position file is then used for one dimensional cell simulations.

\section{Program Structure}

A computer program to model all of the effects of various surface texturing geometries on the enhancement of light trapping in solar cells would be quite complex. The program would have to account for two and three dimensional effects, increase in surface recombination, and other effects which may not even be considered important at this time. The program to be described in this section is not the total answer to the problem of modelling textured solar cells. It is, however, the next step in the development of such a program. The program is capable of modelling a variety of materials and surface structures. Currently, the program supports seven front surface structures, eight back surfaces, and five cover glass texturing geometries. In addition, some of the more common photon loss mechanisms are calculated using the program. 
Some of the other effects that are included in the program include: grid lines parallel to the coordinate axes, loss of photons out the sides of the cell, effect of light passing through a cover glass, both the absorption in and loss of photons out the sides of a cover glass, the effect of a textured cover glass, non-unity back surface reflectance, and front surface reflectance as a function of wavelength. Some of the effects which are not incorporated into the program include reflection as a function of angle of incidence, antireflection coatings, free carrier absorption, and surface pointing errors (imperfect texturization). These effects could be incorporated into the structure of the program with an increase in both memory and computation time. Since it was desired to have a wide base of users, the program was designed to be run on a personal computer. However, with the incorporation of some of the above effects, computation time in most cases may exceed reasonable limits for some users. Fortunately, the program was written using strict FORTRAN 77 standards and therefore should be portable to most mainframes and mini-computers. An alternative to moving the program to a faster computer was to create different output modes. Different modes produce specific information as a compromise to computational speed. An example is calculating a cumulative photon generation number instead of a generation profile as a function of position. The cumulative photon generation number is the total number of photons absorbed but gives no spatial information as to the location of maximum absorption, thus saving time.

\section{Program Flow}

Program flow basically follows the path of the incoming light. An example of program flow is depicted in figure 2-15. For instance, if a cover glass is present, light must pass through the cover glass before it strikes the front of the cell. Every time the 


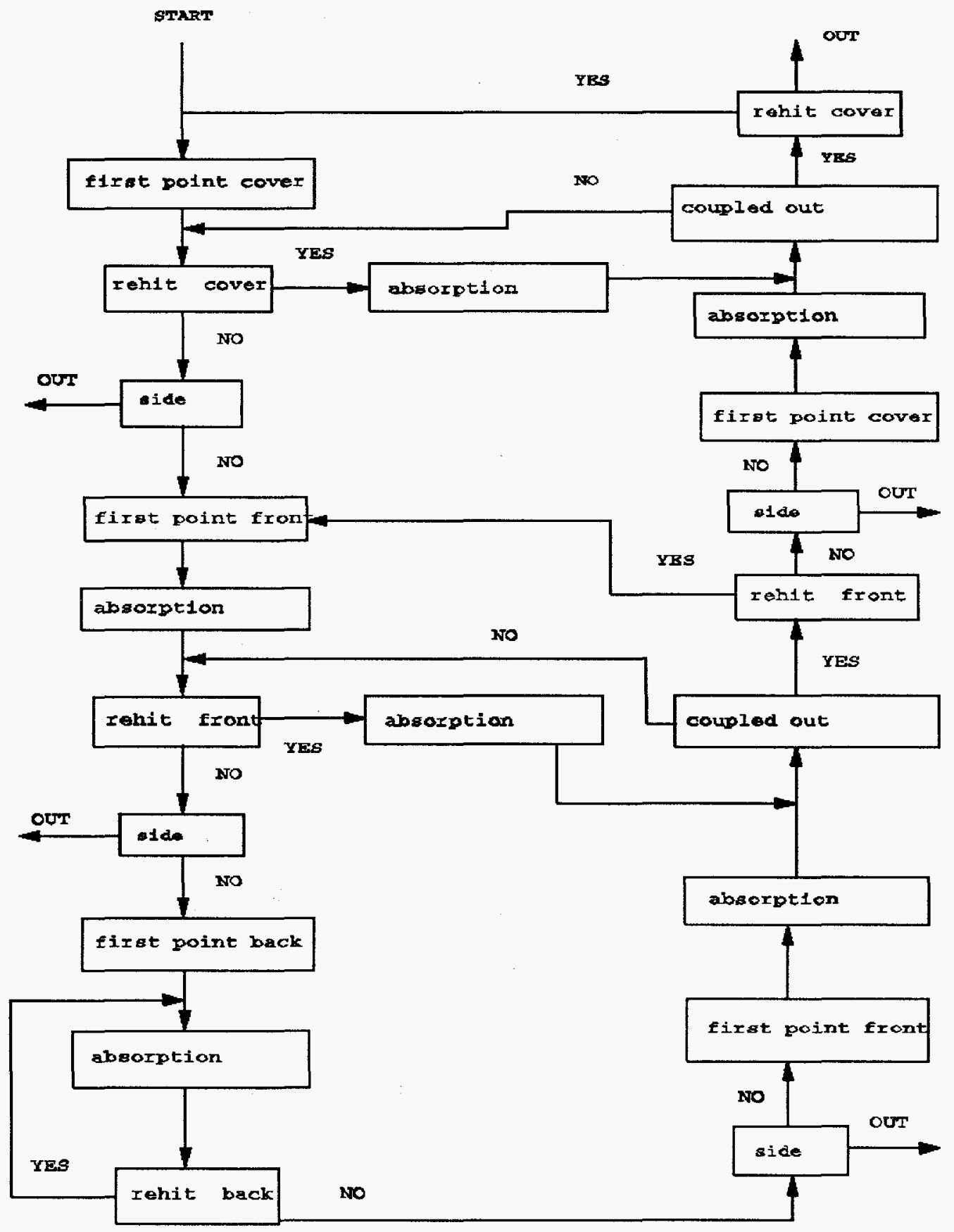

Figure 2-15. Flow chart of raytracing program. At each absorption box, the number of photons is checked against a cutoff. If the number is lower a new ray is started. 
ray strikes an exterior surface, the number of photons is reduced by the amount dictated by the reflection coefficient of the surface. These reflected photons become a daughter ray which will be traced after the trace of the parent ray is completed. As the light passes through the cover glass the path is checked to determine if the side of the cover glass is encountered. If the side of the glass is struck, it is determined if the critical angle has been exceeded. If the angle of incidence is less than the critical angle, the photons are lost and any daughter rays will be traced. If the critical angle has been exceeded, the ray is reflected from the side and tracing continues. Once it has been determined where the photons have hit the top of the cell, by the constrained volume method, a daughter ray is created. This daughter ray contains the photons reflected from the surface of the cell. The remaining portion of photons enter the cell structure and are refracted. After entering the cell, the front surface is re-checked to see if the ray rehits the front from the inside of the cell (only very steep textures). If the front surface is rehit, the critical angle condition must be checked again. Incidence at an angle below the critical angle upon rehitting the front surface creates a daughter ray containing the amount of photons which are refracted out of the cell. If the front surface has not been rehit, then the photons must be travelling towards the back surface. Again the path of the ray is checked to determine, in this case, if the side of the cell is struck. During every process where it is determined that the ray has struck a surface, the distance travelled by the ray is computed and the number of photons in the packet adjusted to reflect the absorption in the material. This process of checking every possible avenue of photon loss mechanism is continued until the ray is either coupled out the cell and it is determined that it may not re-enter, or the number of photons contained in the ray falls below a specified limit. Then if no daughter rays are left, a new parent ray is started. If all of the parent rays for a 
particular wavelength have been simulated, a new set of parent rays is generated at the next wavelength to be modelled. This is continued until all the wavelengths in the spectrum specified have been modelled.

\section{Program Input}

A user-friendly input pre-processor program has been created to isolate the user from much of the mathematics of the program. By running the pre-processor program and answering the questions, a file is created in the proper format for the main program to read. The input program was also written in FORTRAN 77 to be portable between computer systems. However, to provide pop-up menus for the user, ANSI graphical standards have been followed. This limits the use of the program to computers which

\begin{tabular}{|c|c|}
\hline \multicolumn{2}{|c|}{ 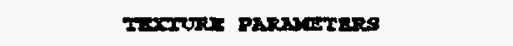 } \\
\hline $\begin{array}{l}\text { F110 wanagerwnt: } \\
\text { Intonafty }(p / \mathrm{em}-2\}= \\
\text { spoctrum: }\end{array}$ & $\begin{array}{l}\text { e110name } \\
1.0 \mathrm{D}-1 \\
\text { AK15d.apC }\end{array}$ \\
\hline 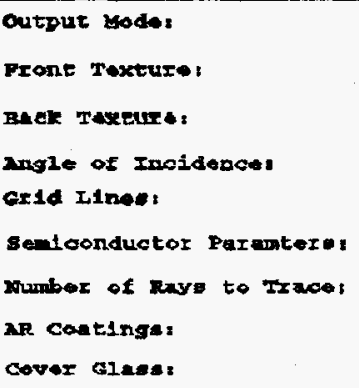 & $\begin{array}{l}\text { OONN } \\
\text { I-TXTRA } \\
\text { FLAT } \\
\text { none } \\
1000 \\
\text { no }\end{array}$ \\
\hline
\end{tabular}

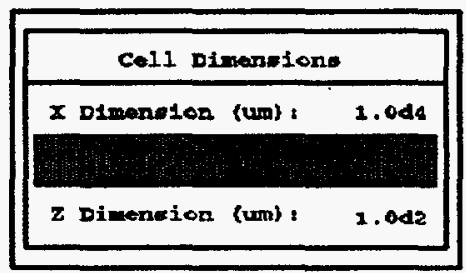

T Dimension (um):

Figure 2-16. Input screen example, shows three levels of menus: the main menu, the cell dimension menu, and the input window. 
are not running other graphical environments. In addition, FORTRAN 77 does not provide the capability of scanning the keyboard for a key stroke, thus requiring a computer dependent assembly language routine. An example of a typical input screen is depicted in figure 2-16. The box on the left of figure $2-16$ shows the main input screen, the reverse video designates the cursor position. The cursor position is changed by using the cursor movement keys or the letters $i$ and $k$. By striking the return and escape keys, the user is presented different levels of menus to input the required data. For instance, if the user strikes return when the cursor is at the position in figure 16 a second menu appears (the box in the upper right) prompting for the cell dimensions. By striking return again an input window at the bottom of the screen opens prompting the user for the specified input (the reverse video in the upper right). Upon entering the information, the program checks for incompatibilities which could cause an error in the main program. If the input is acceptable, the program returns to the previous menu and displays the updated parameter. Once the user has specified all the desired characteristics of the cell to be modeled, a file is created by selecting the "Save File" option under the "File Management" window. The program will then prompt for a file name. Since the main program only requires the ASCll file created by the input program, the input program may be run on a personal computer and the input file ported up to a larger computer. Besides the cell dimensions, the user must specify the light intensity and the spectrum of wavelengths. The spectrums are in the same form as those of PC-1D version 2. The output mode in which the main program is to be run is also an input; the four modes will be explained in the next section. The front and back texture of the cell must also be input along with the corresponding dimensions. Currently the program supports seven front structures. These include: flat, lambertian, slats in the $x$ direction, pyramids, 
tetrahedrons, inverted pyramids, and inverted tetrahedrons. The eight back structures include: flat, lambertian, slats in the $x$ direction, slats in the $y$ direction, positive slats in the $x$ direction, pyramids, tetrahedrons, and square wells. For most structures, the choice of the back texture is independent of the front texture. The user must also enter the angle of incidence of the incoming light. This allows the program to model some important factors. The first is the amount of light trapping in a structure when the system which tracks the sun may be off by a few degrees. Another factor is the amount of light trapping for a fixed system as the sun moves across the sky. This will aid in the determination of a light trapping scheme which is relatively insensitive to the angle of the incoming light. A third factor which may be accounted for by the user input angle of incidence is the effect of a secondary optical element in the solar concentrator system. In the same input menu a small pointing error may be entered to account for the sun not being a point source. At the grid line input, the user is prompted for a file name which contains the geometry of the gridlines to be simulated. Currently, up to 40 grid lines parallel to the coordinate axes may be simulated on a cell. The reflection coefficients as a function of wavelength for a grid line must be specified in the grid file. The program allows the use of two reflection coefficients, for rays which strike the exterior and rays which strike the interior. This accounts for grid lines made of layers of material such as silver (a highly reflective metal) and titanium (a poor reflector). In the semiconductor parameter menu the user is prompted for the semiconductor absorption, index of refraction, reflection coefficient, and back surface reflectance. All of these parameters may be specified as static values or files of values as a function of wavelength. In addition, a thin film dielectric may be simulated on the back surface; i.e. the index of refraction of a material is entered and if the critical angle is violated the ray is coupled out 
of the cell. The number of rays to trace designates the number of parent rays at each wavelength which are to be followed. The higher this number the more statistically accurate the simulation, but at a higher computation cost. The final input is whether a cover glass is present. if it is present, the main menu is enlarged to reflect the information required to define the cover glass. If a cover glass is present, the user must enter the cover glass texture, dimensions, and the material parameters in a manner similar to that of the semiconductor. Currently five surface textures may be simulated: flat, Lambertian, slats in the $x$ direction, slats in the $y$ direction, and pyramids.

\section{Program Output}

Currently there are four output modes supported by the program. These modes are a reflection of the need for different information as a compromise to speed. These output modes include: 1.) quantitative mode, 2.) qualitative mode, 3.) peak-to-peak mode, and 4.) PC-1D mode. These four modes will now be explained.

\section{Quantitative Mode}

The quantitative mode provides the user with the total number of photons absorbed within the cell structure. It does not provide any spatial information. In addition, the number of photons absorbed in the cover glass and those lost out the back, front, and sides of the cell is recorded. The increase in both the front and back surface areas of the cell is provided in the output file. This may be used to estimate the change in open circuit voltage by using the methodology in reference [11]. The percentage of photons absorbed at each wavelength simulated is also output in this mode. This provides the user with an estimate of the spectral response of the cell assuming $100 \%$ 
collection of the photogenerated carriers. For short wavelengths the number of strikes on the front surface may be estimated. For instance, if the front surface reflection coefficient is $10 \%$ and $99.9 \%$ of the short wavelength photons are absorbed then the ray rehits the front surface three times $\left(1-R^{3}\right)$.

\section{Qualitative Mode}

The qualitative mode provides the average number of passes the rays makes before permanently exiting the cell. The path length enhancement is also included in the output, in relation to the path length for a cell with planar surfaces. This mode shows the degree to which the simulated structure traps the light, the more passes the more effective the structure. This mode will provide an estimate of the red response of the cell, since this is the long wavelength region of the spectrum and is very dependent upon long distances to be absorbed. No quantitative information about photon absorption is given in this mode.

\section{Peak-to-Peak Mode}

The peak-to-peak mode gives a generation profile as a function of depth into the cell from the peak of the front structure to the peak of the back surface texture. Using this generation profile in one dimensional cell simulations may be dangerous unless the texture heights are much smaller than the thickness of the cell. If the surfaces of the cell are flat and the cover glass is textured, then this mode will provide a valid one dimensional generation profile for device simulation. This generation profile will be created with a substantial decrease in computer time because the minimum distance routines are bipassed. 


\section{PC-1D Mode}

The PC-1D mode provides the user with an effective one dimensional generation profile as well as a file containing the area as a function of depth into the cell. By using these two files in PC-1D version 2, one dimensional cell simulations may be performed. This will aid in the determination of the best texturing scheme, not only in terms of light trapping but also the compromise to open circuit voltage in the cell structure. 


\section{REFERENCES}

1. B. Dale and H.G. Rudenberg, "High Efficiency Silicon Solar Cells", in Proceedings $14^{\text {th }}$ Annual Power Sources Conf., U.S. Army Signal Research and Development Lab., 1960.

2. A.E. St. John, "Multiple Internal Reflection Structure in a Solicon Detector Which is Obtained by Sandblasting", U.S. Patent 3487 223, 1969.

3. A. Rothwarf and A.M. Barnett, "Design Analysis of the Thin-Film CdS-Cu $\mathrm{C}_{2} \mathrm{~S}$ Solar Cell", IEEE Trans. Elec. Dev., vol. ED-24, pp. 381-387, 1977.

4. H. lida, T. Mishuju, A. Ito, and Y. Hayashi, "The Structure of Natively Textured $\mathrm{SnO}_{2}$ Film and Its Application to an Optical Confinement-Type a-Si:H Solar Cell", IEEE Trans. Elec. Dev., vol. ED-34, pp. 271-276, 1987.

5. J.R. Sites and P.H. Mauk, "Reflection Loss from Polycrstalline CulnSe ${ }_{2}$ Solar Cells", in Proceeding of the 19th IEEE Photovoltaic Specialists Conf., pp. 818-822, 1987.

6. E. Yablonovitch and G.D Cody, "Intensity Enhancement in Textured Optical Sheets", IEEE Trans. Elec. Dev., vol. ED-29, pp. 300-305, 1982.

7. P. Sheng, "Optical Absorption of Thin Film on a Lambertian Reflector Substrate", IEEE Trans. Elec. Dev., vol. ED-31, pp. 634-636, 1984.

8. J. Lodenquai, Theoretical Characteristics of a Solar Cell with a Specific Thin-Film Design", IEEE Trans. Elec. Dev., vol. ED-33, pp. 198-202, 1986.

9. R.A. Arndt, J.F. Allison, J.G. Haynos, and A. Mealenberg, Jr., "Optical Properties of the COMSAT Non-Reflective Cell", in Proceeding of the 11th IEEE Photovoltaic Specialists Conf., pp. 40-43, 1975.

10. C.R. Baraona and H.W. Brandhorst, "V-Grooved Silicon Solar Cells", in Proceeding of the 11th IEEE Photovoltaic Specialists Conf., pp. 44-48, 1975.

11. R.J. Roedel and P.M. Holm, "The Design of Anisotropically Etched III-V Solar Cells", Solar Cells, vol. 11, pp. 221-239, 1984.

12. M.A. Green and P. Campbell, "Light Trapping Properites of Pyramidally Textured and Grooved Surfaces", in Proceeding of the 19th IEEE Photovoltaic Specialists Conf., pp. 912-917, 1987.

13. P. Campbell, S.R. Wenham, and M.A. Green, "Light Trapping and Reflection Control with Tilted Pyramids and Grooves", in Proceeding of the 20th IEEE Photovoltaic Specialists Conf., pp. 713-716, 1988. 
14. T. Uematsu, M. Ida, K. Hane, Y. Hayashi, and T. Saitoh, "A New Light Trapping Structure for Very-Thin, High-Efficiency Silicon Solar Cells", in Proceeding of the 20th IEEE Photovoltaic Specialists Conf., pp. 792-795, 1988.

15. S.P. Tobin, C.J. Keavney, L.M. Geoffroy, and M.M Sanfacon, "Experimental Comparison of Light-Trapping Structures for Silicon Solar Cells", in Proceeding of the 20th IEEE Photovoltaic Specialists Conf., pp. 545-548, 1988.

16. P.A. Basore, "Production Effficiency Goals for Silicon Solar Cells", in Proceeding of the 19th IEEE Photovoltaic Specialists Conf., pp. 905-911, 1987.

17. G.A. Landis, "Thin, Light-Trapping Silicon Solar Cells for Space", in Proceeding of the 20th IEEE Photovoltaic Specialists Conf., pp. 708-712, 1988.

18. P.A. Basore, PC-1D Installation Manual and User's Guide Version 2.1, lowa State Univeristy Research Foundation, Ames, IA, 1989.

19. A. Cuevas, R.A. Sinton, and R.M. Swanson, "Point- and Planar-Junction P-I-N Silicon solar Cells for Concentration Applications. Fabrication, Performance and Stability", in Proceeding of the 21st IEEE Photovoltaic Specialists Conf., pp. $327-$ 332, 1990.

20. J. Zhao, A. Wang, and M.A. Green, "24\% Efficient PERL Structure Silicon Solar Cells", in Proceeding of the 21st IEEE Photovoltaic Specialists Conf., pp. 333-335, 1990. 


\section{THE HYDRODYNAMIC MODEL FOR SEMICONDUCTOR DEVICE SIMULATION}

\subsection{Pros and Cons of Hydrodynamic Model}

Two different types of device modelling are currently used to predict device characteristics. The first is the drift-diffusion model which is computationaly fast but neglects many physical effects such as thermal gradients and ballistic transport. The other type of modelling is the Monte Carlo technique which is physically more detailed but is computationaly intensive and unsuitable for device optimization. Each of these models will be briefly discussed as a prelude to the hydrodynamic model, which is considered to be an intermediate method. The hydrodynamic model is computationaly faster than the Monte Carlo technique but lacks some of the details. On the other hand, the hydrodynamic model is slower than the drift diffusion but it incorporates more physical phenomena that are becoming increasingly important in today's devices. Therefore, the hydrodynamic model can simulate a wide class of devices for which the drift-diffusion and Monte Carlo approaches are inappropriate.

Traditional device modelling is accomplished by solving the drift-diffusion equations self-consistently with Poisson's equation. This technique has long been used as an engineering tool for device design. Unfortunately, with the advent of ultra-small device dimensions, the applicability of this method has been questioned [1]. As device dimensions are decreased, a large spatially nonuniform electric field is created, which produces carrier velocities in excess of the steady state saturation value even at low operating voltages. This velocity overshoot is a result of either of two effects: a difference in energy and momentum relaxation times or carrier scattering into satellite 
valleys or high mobility states. Attempts have been made to accommodate this overshoot effect by making the carrier mobility field dependent $\{\mu(E)\}[2,3]$ or by adding terms to the current equation which depend upon the gradient of the electric field $\{q \mu(E) n L(E) \partial E / \partial x\}[4,5]$. However, Stratton [6] has shown that the field dependent mobility approach is invalid because the mobility should depend upon the average carrier energy. Ballistic transport is still not accounted for by adding the electric field gradient term[4]. The inability to properly account for high field effects results in the prediction of lower carrier velocities than observed. Since the maximum operating frequency of a device depends upon the carrier velocity, this miscalculation can lead to differences between experimental and modelled frequency responses. Also, the carriers are assumed to be in thermal equilibrium with the lattice at all times, so that carrier heating by the field is ignored. Since the rate of carrier diffusion is proportional to carrier temperature, simulations which ignore temperature effects will underestimate diffusion currents [7].

In addition to heating effects, decreasing device dimensions increase the importance of heavy doping effects. According to the rules of MOSFET scaling [8], the doping concentration must be increased to maintain the same output characteristics as the device size decreases. Therefore, as device packing density rises, the doping density must increase to levels which may produce degeneracy. In most simulations these degeneracy effects have not been accurately modeled.

In contrast to the drift-diffusion models, monte carlo simulations are particulate solutions to the Boltzmann transport equation. Therefore, the complex nature of the band structure and scattering events are incorporated into the model. A single carrier, or small ensemble, is followed as it progresses through the device. During its travel, it will 
undergo many different scattering mechanisms. Unfortunately, a large number of particles must be simulated to gain a statistically valid answer. The time scale used in the simulation is very small, which makes it difficult to include carrier generation/recombination processes. The inability to model these bulk processes limits Monte Carlo simulations to devices which do not possess quasi-neutral regions. In addition, if Poisson's equation is to be solved self-consistently, a large amount of computer time is required.

Hybrid schemes involving Monte Carlo simulations in certain regions of a device coupled to drift-diffusion calculations for quasi-neutral regions have been reported $[9,10,11]$. However, the computation time for these simulations is still limited by the monte carlo technique. Regions of applicability of the different models must be identified before simulations begin, making the method non-adaptive. The major problem with this technique is related to satisfying the boundary conditions between different simulation regions.

The hydrodynamic model appears is a compromise between these two methods. It incorporates more physical effects than the drift-diffusion model without a large increase in computation time. Its computation time is much less than monte carlo, but quantum phenomena cannot be modeled. Hot carrier effects and thermal diffusion arise naturally in the derivation of the model. In addition, a lattice heat flow equation is appended easily to the formulation. The scattering events are still treated phenomenologically and may be taken from steady state monte carlo calculations or estimated from measurable quantities. The hydrodynamic model, or some variation of it, has been applied to silicon MOSFETS [12], BJT's [13], and thyristors [14]; GaAs MESFET's [15,16], logic elements [17], and HEMT's [18]; and AIGaAs/GaAs HBT's [19,20,21]. Some of the areas in which 
this model has not been applied to date include: solar cells under concentration, thermoelectric devices, avalanche photodiodes, and power rectifiers, where its application is expected to be important.

In the next section the transport model derivation will be discussed. Where appropriate, conditions will be imposed to show that the hydrodynamic model reduces to the drift-diffusion model. Section 3 highlights the need for such a model by exposing hot carrier or temperature effects in different devices. Numerical results of hot carrier models by other authors is presented in section 4 . The assumptions made in these models will be discussed. A detailed account of the derivation of a general moment equation is included in the appendix and the continuity and energy moment equations are provided as examples. 


\subsection{Development of Transport Model}

\section{Assumptions of BTE}

Since both the hydrodynamic and drift-diffusion models are based entirely on the Boltzmann transport equation (BTE), some discussion of the transport equation is in order. The BTE is a valid representation of the time dependent motion of charge carriers and energy provided the following assumptions hold:

1) The number of carriers in the device is large enough that a statistical distribution function applies [22].

2) Quantum mechanical phenomena are included in the effective mass and scattering coefficient terms [22].

3) Individual electrons are considered to be uncorrelated, i.e. there is little carrier-carrier interaction [3].

4) The scattering probability is independent of external forces and collisions are instantaneous [3].

5) Forces applied to the electron wavepacket do not vary appreciably over the wavelength of the packet, i.e. $10 \mathrm{~nm}$ [23].

In most devices, these assumptions can be justified. Only in quantum structures where distinct energy states arise will these assumptions begin to fail. The reason for this inability to model quantum phenomena is due to the semi-classical nature of the equation; both position and momentum of the particles are assumed to be known at all times. If the quantum mechanical system can be considered separate, then the bulk and quantum systems may be coupled as done by Widiger for HEMT's [18]. The semiclassical form of the BTE used is shown in (1) and can actually be derived from the 
quantum mechanical Louiville equation [24].

$$
(\partial f / \partial t)+v \cdot \nabla f+F \cdot \nabla_{k} f=(\partial f / \partial t)_{c}
$$

This is a continuity equation representing all of the phenomena which affect the

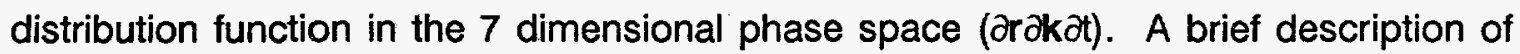
each term will now be undertaken. The first term represents the time rate of change of the distribution function. The second term shows the change in the distribution due to carriers diffusing under their own velocity into or out of the volume. Those carriers exiting or entering the volume by a drift field caused by some applied force is represented by the third term. The last term indicates how many carriers leave or enter the volume due to scattering events. As noted in the assumptions most of the quantum mechanical effects are included in the scattering term. Moments of the BTE are obtained by multiplying $(3-1)$ by the quantity to be conserved and integrating over all $\mathbf{k}$ space. To completely describe the system, an infinite set of moments must be calculated because each moment will contain a moment of the next higher order. Therefore, at some point an approximation must be made for the highest moment. In the derivation to follow it has been assumed that the $5^{\text {th }}$ moment can be written as an energy squared term. In order to avoid adding complexity to the derivation of the moments, the following observations can be made about the scattering term:

1) The scattering term must vanish in equilibrium (evaluate to zero) for all moments.

2) When a stimuli disturbs the distribution, the scattering events must return the system to equilibrium.

The simplest form to accomplish these two criteria is 


$$
(\partial f / \partial t)_{c}=-\left(f-f_{o}\right) / \tau .
$$

The quantity $\tau$ is the relaxation time, and (3-2) is the relaxation time approximation. Substitution of this back into (3-1) produces the final form of the Boltzmann transport equation to be used

$$
(\partial f / \partial t)+v \cdot \nabla f+F \cdot \nabla_{k} f=-\left(f-f_{o}\right) / \tau .
$$

F is given by $(-\nabla €-\nabla W)$ for electrons [25]. To simplify the derivation, the crystal structure was assumed to be cubic with ellipsoidal energy surfaces in $\mathbf{k}$ space. This reduces the effective mass tensor to a vector and the temperature tensor to a scalar [26]. In all cases, moments can be generated for holes by a similar derivation. If the semiconductor has satellite valleys then moment equations for each valley must be formulated. The equations for different valleys would then be coupled by the scattering terms.

The hydrodynamic model is created by taking the first four moments of the Boltzmann transport equation (BTE), similar to the drift-diffusion model which takes the first two moments. Up to this point both models contain the same assumptions. Energy balance equations for semiconductors were first applied to homogeneous material with a parabolic band structure by Stratton [6] and further extended by Blotekjaer to include general distributions [27]. Both of these derivations expanded their final distribution function in spherical harmonics and added terms heuristically; i.e. heat flow. Rudan and Odeh's [26] derivation used a general distribution function, but reduced their equations to Boltzmann statistics in the end. The first attempt to relax the homogenous material constraint and keep Fermi-Dirac statistics was reported by McAndrew et al [28]. This 
derivation properly accounted for the terms added in earlier derivations (heat flow) and eliminated one variable in the energy equation. But, it also added terms in the carrier continuity equation which do not appear in any other derivation. Azoff [29] also generalized the derivation by including inhomogeneous material and Fermi-Dirac statistics; however, the fourth moment was only approximated. In a later paper, Azoff relaxed the parabolic band approximation [30]. Unfortunately, when the parabolic band approximation is relaxed the equations cannot be easily expressed in a conservation form. This loss of the conservation form leads to difficulties in applying efficient numerical methods to the equations.

\section{Particle Balance Equation}

The carrier continuity equation is obtained by taking the first moment of (3-3), which is accomplished by multiplying it by 1 and integrating over all $k$ space. The resulting equation is

$$
(\partial n / \partial t)+\nabla \bullet(n v)=-R+G
$$

Here $R$ and $G$ are the recombination and generation rates, respectively. For semiconductors R is usually accounted for by Shockley-Read-Hall, Auger, and band-toband recombination. These must be added heuristically since they do not arise naturally in the BTE. The first term denotes the time rate of change in the carrier concentration. The second term represents the change in carrier concentration due to flow. This equation is the same for both the hydrodynamic and drift-diffusion models. In the literature, McAndrew et al [28] recover the following continuity equation 


$$
(\partial n / \partial t)+m_{c e} \nabla \cdot\left(n v / m_{c e}\right)=-R+G .
$$

The second term is different because they did not cancel out the gradient of the conductivity effective mass arising from the third term in (3-3) with the one coming from the second term in (3-3).

\section{Particle Flux Balance Equation}

The second moment produces the current equation (conservation of momentum), with $v$ being the conserved quantity. The general form of the equation is given by

$$
-n v=\tau_{2}(\partial(n v) / \partial t)+(2 / 3) \nabla(n<W>)+n \nabla e-n<W>\nabla \ln \left(m^{*}\right)[29]
$$

Until a distribution function is assumed, (6) cannot be used in device modelling. By using the Fermi-Dirac distribution function the resulting form is

$$
\begin{aligned}
& -n v=\tau_{2}(\partial(n v) / \partial t)+\sum\left(\tau_{2} / m_{i}\right) \hat{a}_{i} \hat{a}_{i} \cdot\left[k_{b} T_{e}\left(F_{y_{2}}\left(\eta_{c}\right) / F_{y_{2}}\left(\eta_{c}\right)\right) \nabla n+\right. \\
& n \nabla \varepsilon_{c}-n \nabla \chi-1 / 2 n k_{b} T_{e}\left(F_{y_{2}}\left(\eta_{c}\right) / F_{-1 / 2}\left(\eta_{d}\right)\right) \sum \nabla m_{i} / m_{i}+ \\
& \left.(5 / 2) n k_{b}\left(F_{3 / 2}\left(\eta_{c}\right) / F_{y_{2}}\left(\eta_{d}\right)\right)\left(1-(3 / 5)\left(F_{y_{2}}\left(\eta_{d}\right) F_{y_{2}}\left(\eta_{d}\right) /\left(F_{3 / 2}\left(\eta_{d}\right) F_{-1 / 2}\left(\eta_{c}\right)\right)\right)\right) \nabla T_{e}\right]
\end{aligned}
$$

Where $\hat{a}_{i}$ is a unit vector in the $x, y$, or $z$ direction and $F_{-y_{2}}\left(\eta_{d}\right), F_{y_{2}}\left(\eta_{c}\right)$ and $F_{3 / 2}\left(\eta_{c}\right)$ are the Fermi integrals of different orders, with $\eta_{c}$ as the reduced Fermi energy. The first term on the RHS is the transient response of the current, and is usually neglected because the current reacts almost instantaneously to an applied force. The multiplicative term $\left(\left(\tau_{2} / m_{i}\right) \hat{a}_{i} \hat{a}_{i}\right)$ in the sum is usually defined, after multiplying by $q$, as the mobility. Notice 
in this derivation the mobility will be direction dependent similar to the result obtained by Kishore [31]. The first term in the sum on the right hand side is the diffusion component. Under non-degenerate conditions the Fermi integrals reduce to exponentials and the ratio evaluates to 1 which results in the normal diffusion term, $k_{b} T_{e} \mu \nabla n / q$. The next term in the sum is due to the gradient in the conduction band, which includes the potential $\psi$ and any heavy doping effects. The next term, $\nabla \chi$, is due to the gradient in the electron affinity. This term will only be important in graded gap and heterojunction devices. The fourth term in the sum indicates that electrons will move toward a region with a larger effective mass. The second sum in the effective mass term arises because the crystallographic axes do not necessarily have to match to the axes of cartesian coordinates. In several drift diffusion simulations [32,33] the effective mass term has been written as $1 / 2 n k_{b} T_{e}\left(F_{y_{2}} / F_{-y_{2}}\right) \nabla N_{c} / N_{c}$. However, as pointed out in [34] this only applies for non-degenerate materials. The final term in the summation is the result of a force which will tend to move the electron towards sections of the material with a cooler electron temperature. This effect is utilized in thermal electric devices. In order to recover the commonly used drift-diffusion equations for homojunctions, the following simplifications are made:

1) No temperature gradients exist, the last term in the sum is zero.

2) A homogeneous material with constant effective mass and uniform electron affinity is present, therefore the third and fourth terms of the sum are zero.

3) The system is non-degenerate, all Fermi integrals reduce to exponentials (Boltzmann factors) and ratios of the Fermi integrals evaluate to one.

4) The transient response of the current is zero, this implies an instantaneous reaction of the current to an applied force. 
The result of all of these assumptions leads to

$$
-n v=\mu k_{b} T_{e} \nabla n / q+n \mu v \varepsilon_{c}
$$

which is the commonly used drift diffusion equation.

\section{Energy Balance Equation}

The $3^{\text {rd }}$ moment will produce an energy conservation equation, multiply (3) by $\mathrm{W}$ and integrate over all $\mathbf{k}$ space. The resulting conservation relation is

$$
\partial(n W) / \partial t+\nabla \bullet(n q)+n v \cdot \nabla \epsilon_{c}-n v \bullet \nabla \chi=-(n W-n W o) / \tau_{3}
$$

Where $n q$ is the energy flux and $\mathrm{nW}_{\mathrm{o}}$ is the equilibrium energy. The first term is the time rate of change in the average energy. The third and fourth terms reflect the amount of energy the carriers gain due to the field and change in electron affinity. In some instances $[14,35,36]$ the third term is called the joule heating and appended directly to the lattice heat flow equation. This is incorrect because the carriers gain the energy from the field, not the lattice. Since the electrons do not have to be in thermal equilibrium with the lattice at all times the joule heat is not applied directly to the lattice. The carriers may either drift or diffuse to another region of the device before thermalizing. The term on the RHS reflects the amount of energy gained or lost due to recombination or generation and the energy gained or lost due to scattering with the lattice. This term couples the electron temperature to the lattice and hole temperatures. At this point in the derivation discrepancies arise in the form which $W$ should take on arise. If the distribution function 
was assumed to be a displaced Maxwellian, then the form of $W$ is

$$
W=(1 / 2) m_{e} V^{2}+(3 / 2) k_{b} T
$$

The displaced Maxwellian was used in early derivations which used spherical harmonics to expand the distribution function. However, if the distribution function is Fermi-Dirac, then the form is

$$
W=(3 / 2) k_{b} T_{0}\left(F_{3 / 2}\left(\eta_{d}\right) / F_{1 / 2}\left(\eta_{d}\right)\right)
$$

The reason for the discrepancy lies in the shape of the distribution function. When the Maxwellian is shifted to a higher energy the center of mass of the function moves, but the states of the lower energy are still populated. In the case of the Fermi-Dirac distribution the lower energy states actually become depopulated [37], figure 3-1 [38]. Models which use equation (3-10) frequently ignore the kinetic portion, justifying this by assuming that it is much smaller than the thermal component.

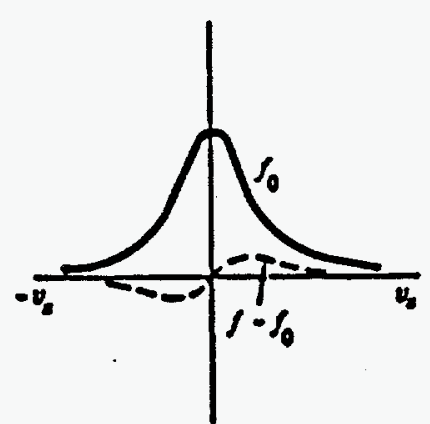

(a)

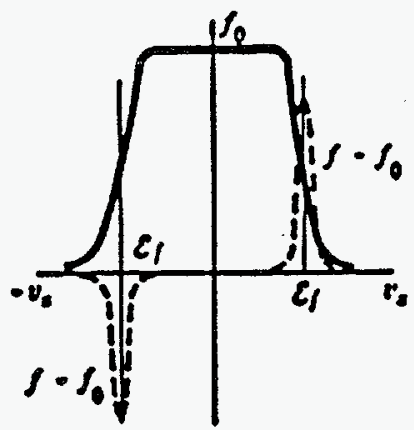

(b)

Figure 3-1. Change in distribution function in the presence of an applied field:

a) Boltzmann; b) Fermi-Dirac [38]. 
Turning to the term on the RHS of the energy balance equation, Cook [13] applied an analytic model for the energy source $\backslash$ sink for Si but did not account for the lattice heating. Szeto [39] expanded Cook's model and recognized that the term should be coupled to the lattice heat flow equation. The form used by Szeto also couples the electron and hole temperatures as seen below

$$
\begin{aligned}
& \left(n W-n W_{o}\right) / \tau_{3}=(3 / 2) k_{b}\left(\left(n / \tau_{n 1}\right)\left(T_{e}-T_{1}\right)+\left(p / \tau_{p 1}\right)\left(T_{h}-T_{1}\right)\right) \\
& +(3 / 2) k_{b}\left(T_{e}-T_{p}\right) p n\left(1 /\left(p_{o} \tau_{n 2}\right)-1 /\left(n_{o} \tau_{p 2}\right)\right)+E_{g} R \text { [39] }
\end{aligned}
$$

The energy balance equation presented in (3-9) is the same as presented elsewhere except the third and fourth terms are a result of an expansion [40]. For a homogeneous material the fourth term on the right is zero. If $n v \bullet \nabla \varepsilon_{c}$ and $\left(n W-n W_{0}\right) / \tau$ are rewritten as $\mathbf{J} \cdot \nabla \epsilon_{\mathrm{c}}$ and $\mathrm{nB}(\mathrm{T})$ respectively, then an equation similar in form to that obtained by Stratton [6] and Widiger [18] is recovered. Instead of using an analytical expression for $\mathrm{nB}(\mathrm{T})$ as in [13] and [6], Widiger [18] and others use steady state monte carlo simulations to estimate the energy relaxation effects.

\section{Energy Flux Balance Equation}

The $4^{\text {th }}$ moment provides the energy flux conservation equation. It is obtained by multiplying (3-3) by $W v$ and integrating over all $k$ space. The resulting equation including Fermi-Dirac statistics and variable bandgap materials is 


$$
\begin{gathered}
-n q=\tau_{4}(\partial(n q) / \partial t)+\sum\left(5 \tau_{4} / 2 m_{\mathrm{i}}\right) \hat{a}_{\mathrm{i}} \hat{a}_{\mathrm{i}} \cdot\left[n k_{\mathrm{b}} T_{e}\left(F_{3 / 2}\left(\eta_{d}\right) / F_{1 / 2}\left(\eta_{d}\right)\right) \nabla \epsilon_{c}-\right. \\
n k_{b} T_{e}\left(F_{3 / 2}\left(\eta_{d}\right) / F_{1 / 2}\left(\eta_{d}\right)\right) \nabla \chi+n\left(k_{b} T_{e}\right)^{2}\left(F_{3 / 2}\left(\eta_{d}\right) / F_{y^{2}}\left(\eta_{d}\right)\right) \nabla n \\
-1 / 2 n\left(k_{b} T_{e}\right)^{2}\left(F_{3 / 2}\left(\eta_{d}\right) / F_{1 / 2}\left(\eta_{d}\right)\right) \sum \nabla m_{i} / m_{i} \\
+(7 / 2)^{*} n k_{b}{ }^{2} T_{e}\left(F_{5 / 2}\left(\eta_{d}\right) / F_{1 / 2}\left(\eta_{d}\right)\right)\left(1-(3 / 7)\left(F_{y_{2}}\left(\eta_{d}\right) F_{3 / 2}\left(\eta_{d}\right) / F_{5 / 2}\left(\eta_{d}\right) F_{-1 / 2}\left(\eta_{d}\right)\right) \nabla T_{e}\right]
\end{gathered}
$$

As in the case of the current equation the multiplier in the sum will make the energy flow direction dependent. Also, only if $\tau_{4}$ is equal to $\tau_{2}$ is it valid to set $q \tau_{4} / m_{i}$ equal to the electron mobility. If the material system in question is non-degenerate, then $\Delta$ and $\delta$ are defined as [6]

$$
\delta(T)=\Delta(T)=\left(5 \tau_{4} / 2 \tau_{2}\right)
$$

then equation (3-13) may be rewritten in the following form, neglecting the transient term

$$
-n q=+\delta(T) k_{b} T n v+\Delta(T) k_{b} \nabla T .
$$

This is basically equation (3-8) in [6], but the composition dependence is included in our formulation. Equations in the form of (3-15) are also used in several other references $[13,16,41]$. Usually the variables $8(T)$ and $\Delta(T)$ are fixed to certain constant values of order 1 . Since this approximation may not be valid at elevated carrier temperatures or with carrier degeneracy, the full form of equation (3-11) should be utilized. The explanations of each individual term in (3-13) follow along the same lines as that of the current equation (3-7), and therefore will not be repeated.

The final form of the system of equations can now be given as 
Electron continuity:

$$
(\partial n / \partial t)+\nabla \cdot(n v)=-R+G
$$

Hole continuity:

$$
(\partial p / \partial t)+\nabla \cdot(p v)=-R+G
$$

Electron energy balance:

$$
\partial\left(n W_{n}\right) / \partial t+\nabla \cdot\left(n q_{n}\right)+n v \cdot \nabla \varepsilon_{c}-n v \cdot \nabla \chi=-\left(n W_{n}-n W_{0}\right) / \tau_{3 n}
$$

Hole energy balance:

$$
\partial\left(p W_{p}\right) / \partial t+\nabla \bullet\left(p q_{p}\right)+p v \bullet \nabla \varepsilon_{v} \cdot p v \bullet \nabla \chi-p v \bullet \nabla E_{g}=-\left(p W_{p}-p W_{o}\right) / \tau_{3 p}\left(3-1{ }^{\prime}\right.
$$

Poisson's equation:

$$
\nabla \bullet\left(\epsilon_{r} \nabla \psi\right)=\left(q / \epsilon_{o}\right)\left(p-n+N_{d}^{+}-N_{a}\right)
$$

Lattice heat flow:

$$
\rho c_{p}\left(\partial T_{1} / \partial t\right)+\nabla\left(\kappa \nabla T_{1}\right)=\left(n W_{n}-n W_{o}\right) / \tau_{3 n}+\left(p W_{p}-p W_{o}\right) / \tau_{3 p}
$$

Equations (3-20) and (3-21) are Poisson's equation and the lattice heat flow equation respectively. The terms introduced in (3-21), $\rho$ and $c_{p}$, are the material density and heat capacity. Notice that losses in carrier energy in (3-18) and (3-19) are generation terms in (3-21). Also, note that since this derivation used the Fermi-Dirac distribution the forms of $W$ are be given by

$$
\begin{aligned}
& W_{n}=(3 / 2) k_{b} T_{e}\left(F_{3 / 2}\left(\eta_{c}\right) / F_{1 / 2}\left(\eta_{c}\right)\right) \\
& W_{p}=(3 / 2) k_{b} T_{p}\left(F_{3 / 2}\left(\eta_{v}\right) / F_{1 / 2}\left(\eta_{v}\right)\right)
\end{aligned}
$$

This yields the six equations in the six unknowns of $n, p, \psi, T_{e}, T_{h}$, and $T_{1}$. 
The auxiliary equations which must accompany these are:

Electron flux:

$$
\begin{aligned}
& -n v_{n}=\tau_{2 n}\left(\partial\left(n v_{n}\right) / \partial t\right)+\sum\left(\tau_{n 2} / m_{e j}\right) a_{i} \hat{a}_{i} \cdot\left[k_{b} T_{e}\left(F_{y_{2}}\left(\eta_{d}\right) / F_{y_{2}}\left(\eta_{d}\right)\right) \nabla n+n \nabla e_{c}-n \nabla x\right. \\
& -1 / 2 n k_{b} T_{e}\left(F_{1 / z}\left(\eta_{d}\right) / F_{-1 / 2}\left(\eta_{d}\right)\right) \sum \nabla m_{e i} / m_{e i} \\
& \left.+(5 / 2)^{*} n k_{b}\left(F_{3 / 2}\left(\eta_{d}\right) / F_{y_{2}}\left(\eta_{d}\right)\right)\left(1-(3 / 5)\left(F_{y_{2}}\left(\eta_{d}\right) F_{y_{2}}\left(\eta_{d}\right) /\left(F_{3 / 2}\left(\eta_{c}\right) F_{-y_{2}}\left(\eta_{d}\right)\right)\right)\right) \nabla T_{a}\right]
\end{aligned}
$$

Hole flux:

$$
\begin{aligned}
& -p v_{p}=\tau_{2 p}\left(\partial\left(p v_{p}\right) / \partial t\right)+\sum\left(\tau_{p 2} / m_{p i}\right) \hat{a}_{i} \hat{a}_{i} \cdot\left[k_{b} T_{p}\left(F_{i k p}\left(\eta_{v}\right) / F_{t / p p}\left(\eta_{v}\right)\right) v p+p \nabla \varepsilon_{v}-p \nabla \chi\right. \\
& -p v E_{g}-1 / 2 p k_{b} T_{p}\left(F_{1 / p p}\left(\eta_{v}\right) / F_{-1 / p p}\left(\eta_{v}\right)\right) \sum \nabla m_{p i} / m_{p i} \\
& \left.+(5 / 2)^{*} \mathrm{pk}_{\mathrm{b}}\left(\mathrm{F}_{3 / 2}\left(\eta_{\mathrm{v}}\right) / F_{y_{2}}\left(\eta_{\mathrm{v}}\right)\right)\left(1-(3 / 5)\left(F_{y_{2}}\left(\eta_{\mathrm{v}}\right) F_{v_{k} / 2}\left(\eta_{v}\right) /\left(F_{3 / 2}\left(\eta_{v}\right) F_{-y_{2}}\left(\eta_{v}\right)\right)\right)\right) \nabla T_{e}\right] \quad(3-25)
\end{aligned}
$$

Electron energy flux:

$$
\begin{gathered}
-n q_{n}=\tau_{4 n}\left(\partial\left(n q_{n}\right) / \partial t\right)+\sum\left(5 \tau_{4 n} / 2 m_{e i}\right) \hat{a}_{i} a_{i} \cdot\left[n k_{b} T_{e}\left(F_{3 / 2}\left(\eta_{d}\right) / F_{1 / 2}\left(\eta_{d}\right)\right) \nabla \epsilon_{c}\right. \\
-n k_{b} T_{e}\left(F_{3 / 2}\left(\eta_{d}\right) / F_{1 / 2}\left(\eta_{d}\right)\right) \nabla \chi+n\left(k_{b} T_{e}\right)^{2}\left(F_{3 / 2}\left(\eta_{d}\right) / F_{1 / 2}\left(\eta_{d}\right)\right) \nabla n \\
-1 / 2 n\left(k_{b} T_{e}\right)^{2}\left(F_{3 / 2}\left(\eta_{c}\right) / F_{1 / 2}\left(\eta_{d}\right)\right) \sum \nabla m_{e i} / m_{e i} \\
+(7 / 2)^{*} n k_{b}{ }^{2} T_{e}\left(F_{5 / 2}\left(\eta_{c}\right) / F_{1 / 2}\left(\eta_{c}\right)\right)\left(1-(3 / 7)\left(F_{1 / 2}\left(\eta_{c}\right) F_{3 / 2}\left(\eta_{d}\right) /\left(F_{5 / 2}\left(\eta_{d}\right) F_{y / 2}\left(\eta_{d}\right)\right)\right) \nabla T_{e}\right]
\end{gathered}
$$

Hole energy flux:

$$
\begin{aligned}
& -p q_{p}=\tau_{4 p}\left(\partial\left(p q_{p}\right) / \partial t\right)+\sum\left(5 \tau_{4} / 2 m_{p i}\right) \hat{a}_{i} \hat{a}_{i} \cdot\left[p k_{b} T_{p}\left(F_{3 / 2}\left(\eta_{v}\right) / F_{1 / 2}\left(\eta_{v}\right)\right) \nabla \epsilon_{c}\right. \\
& \text { - } p k_{b} T_{p}\left(F_{3 / 2}\left(\eta_{v}\right) / F_{y_{2}}\left(\eta_{v}\right)\right) \nabla \chi-p k_{b} T_{p}\left(F_{3 / 2}\left(\eta_{v}\right) / F_{y_{2}}\left(\eta_{v}\right)\right) \nabla E_{g} \\
& +p\left(k_{b} T_{p}\right)^{2}\left(F_{3 / 2}\left(\eta_{v}\right) / F_{1 / 2}\left(\eta_{v}\right)\right) v p-1 / 2 p\left(k_{b} T_{p}\right)^{2}\left(F_{3 / 2}\left(\eta_{v}\right) / F_{1 / 2}\left(\eta_{v}\right)\right) \sum v m_{p i} / m_{p i}+ \\
& \left.+(7 / 2){ }^{*} \mathrm{pk}_{\mathrm{b}}{ }^{2} \mathrm{~T}_{\mathrm{p}}\left(F_{5 / 2}\left(\eta_{v}\right) / F_{1 / 2}\left(\eta_{v}\right)\right)\left(1-(3 / 7)\left(F_{1 / 2}\left(\eta_{v}\right) F_{3 / 2}\left(\eta_{v}\right) /\left(F_{5 / 2}\left(\eta_{v}\right) F_{-1 / 2}\left(\eta_{v}\right)\right)\right)\right) \nabla T_{p}\right] \text {. }
\end{aligned}
$$


This is the full system which must be solved for the hydrodynamic model. Normally the transient terms in equations (3-24) through (3-27) are assumed to be negligible, similar to the drift-diffusion model. By substituting equations (3-24) through (3-27) into equations (3-20) through (3-22), conservation equations for the six unknown parameters are obtained. The rationale for retaining the Fermi functions of different order is illustrated in figure 3-2. As degeneracy is approached the functions begin to separate. Since the ratio of the functions appears in all of the auxiliary equations, setting these ratios to one is not acceptable.

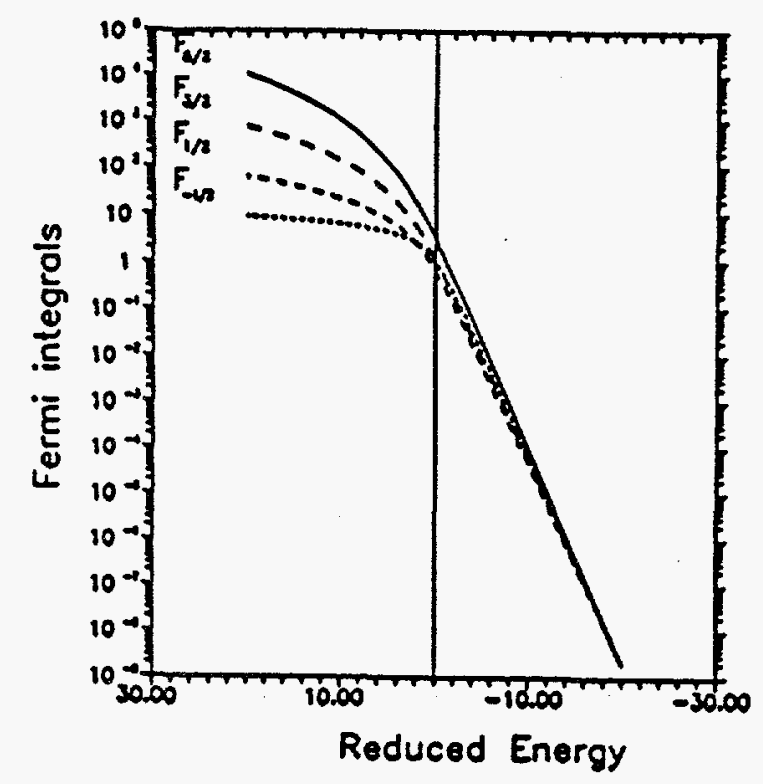

Figure 3-2. Half order Fermi integrals over a reduced energy range from -20 to 20 [42]. 
3.3. Potential Application to Solar cells, Thyristors, and HBT's

\section{Solar Cells}

The principle source of the photovoltaic action in solar cells is the junction or barrier of the device. As the photogenerated charge carriers cross these regions their direction is coincident with falling bandedges, see figure 3-3. From the figure it is seen that the carriers are "hot" and lose energy as they traverse the barrier; i.e. they thermalize. Also from the figure, it is observed that hot carriers are intrinsic to solar cells due to the direction of the current flow. Hot carrier effects may be more pronounced in concentrator designs due to device heating and high current densities. Lattice heating is a direct effect of the interaction of carriers and the lattice. In heterojunction solar cells effective forces, changes in density of states and electron affinities, could lead to junction field values of the order of $10^{4} \mathrm{~V} / \mathrm{cm}$ which produces effective electron temperatures of $1000 \mathrm{~K}$ [43]. This large disparity between carrier and lattice temperatures results in the following effects [44]:

1) the Einstein relation in its simple form no longer holds;

2) the carrier velocity saturates, (velocity saturation is a direct effect of lattice and carrier interaction, if hot carrier effects were ignored the velocity would be proportional to the electric field);

3) the current-voltage relationship must include $T_{e}$ (electron temperature), which is a function of current.

By this last effect any change in carrier temperature, which can be significantly different 
from the lattice temperature, could modify the current-voltage characteristics [45]. Hot carriers can also degrade the front surface passivation layer of a solar cell by producing defects at the semiconductor-passivation layer interface which leads to an increase the front surface recombination. This increase in the front recombination could lead to a decrease in cell efficiency, as seen in the Stanford point-contact cell [46].

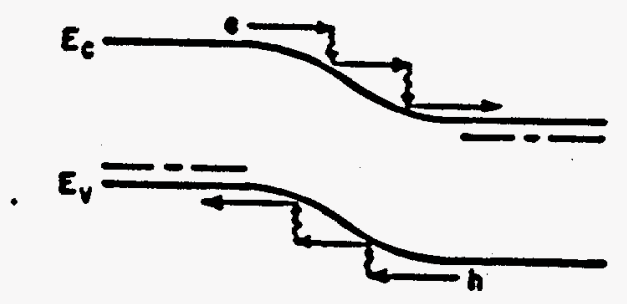

Figure 3-3. Thermalization of hot photogenerated carriers crossing a P-N junction [45].

Thyristors

The dissipation of power in thyristors limits both the steady state and surge current ratings of the device. Power dissipation in certain regions can lead to increased device temperatures (above $125 \mathrm{C}$ ) which can result in random firing of the device [47]. Also in some instances the forward current may be concentrated in a small part of the cross-sectional area of the device when the gate is triggered. Localized heating due to large temperatures from these affects could damage the device. Power dissipation also results from: [48]

1) switching loss; 
2) forward conduction loss;

3) blocking loss;

4) triggering losses.

Only in high frequency applications will the triggering and switching losses become significant. Also at high frequencies the transient component of the lattice heat flow will begin to affect the device temperature. Of the four losses, forward conduction is usually the main source of junction heating. Under steady state conditions, assuming the instantaneous thermaliztion of the carriers, the lattice temperature rise of the thyristor is determined by the product of the power dissipation (Joule heating term) and the thermal resistance of the device and its cooling system:

$$
\Delta T=P \cdot R_{T H}
$$

Where $\Delta T$ is the change in lattice temperature, $P$ is the power dissipated and $R_{T H}$ is the thermal resistance. This simple equation can also be applied to other devices where carrier thermalization is assumed. If the thermal resistance is assumed to be low then the change in device temperature will be minimal. However, if the device is isolated the temperature rise could lead to failure of the device. If the assumption of instanteous thermaliztion of the carriers is invalid then the full system of hydrodynamic equations must be solved to adequately describe the device physics

HBT's

The transit time of the charge carriers across the device determines the frequency capabilities of heterojunction transistors. In order to increase the maximum frequency response either the device must be made smaller or the drift velocity of the carriers must 
be increased. Unfortunately, decreasing the device dimensions leads to a loss in the power handling capabilities of the device. In normal BJT's the carrier drift velocity is proportional to the electric field. This trend continues until the saturation velocity is attained, after which an increase in the field does not increase the velocity. In an HBT, the change in material can produce large discontinuous fields which can produce velocity overshoot. This overshoot, velocities in excess of the saturation velocity, decreases the transit time of the carriers. An approximation for the upper frequency limit is $1 / \tau_{t}$, where $\tau_{t}$ is the transit time [49]. MODFET's with 0.25 micron gates have been produced with 2 picosecond transit times, corresponding to frequency limits of $80 \mathrm{GHz}$ [50].

\subsection{Preliminary Results of Hydrodynamic Model}

Several devices under equilibrium conditions have been successfully modelled using the basic hydrodynamic program. Figure 3-4 is an example of the equilibrium solution for two Si diodes: a heavily doped emitter and a lightly doped device. These devices were simulated with both Fermi-Dirac and Boltzmann statistics. From the figure it is clearly seen that the results for these two statistics are identical for the lightly doped device, while in the case of the heavily doped device the Fermi-Dirac statistics produce a different solution. A comparison with PC-1D [51] is also included in the figure for verification of the program. Figure $3-5$ depicts the effect of interface charge in a uniformly doped material, at each material interface the user is allowed to specify the trapped charge. The ability to specify an interface charge is due to the integrated nature of the numerical method employed. The final figure, Figure 3-6, shows the equilibrium solution

for a heterojunction device $\left(\mathrm{Al}_{0.8} \mathrm{Ga}_{0.2} \mathrm{As} / \mathrm{GaAs}\right)$ with and without interface charge. 


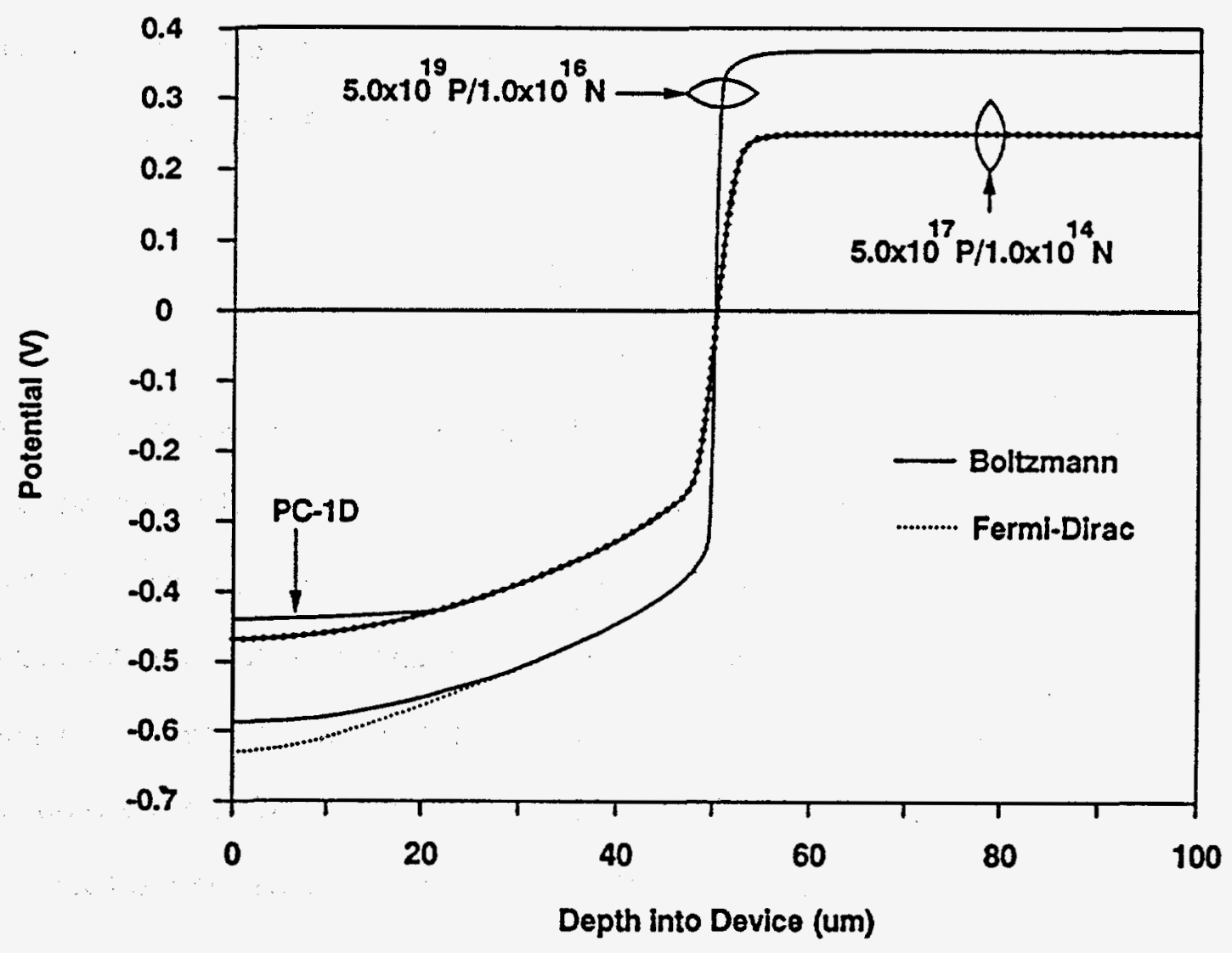

Figure 3-4. Equilibrium solution for two Si diodes, both Fermi-Dirac and Boltzmann statistics were used, comparison with PC-1D is provided. 


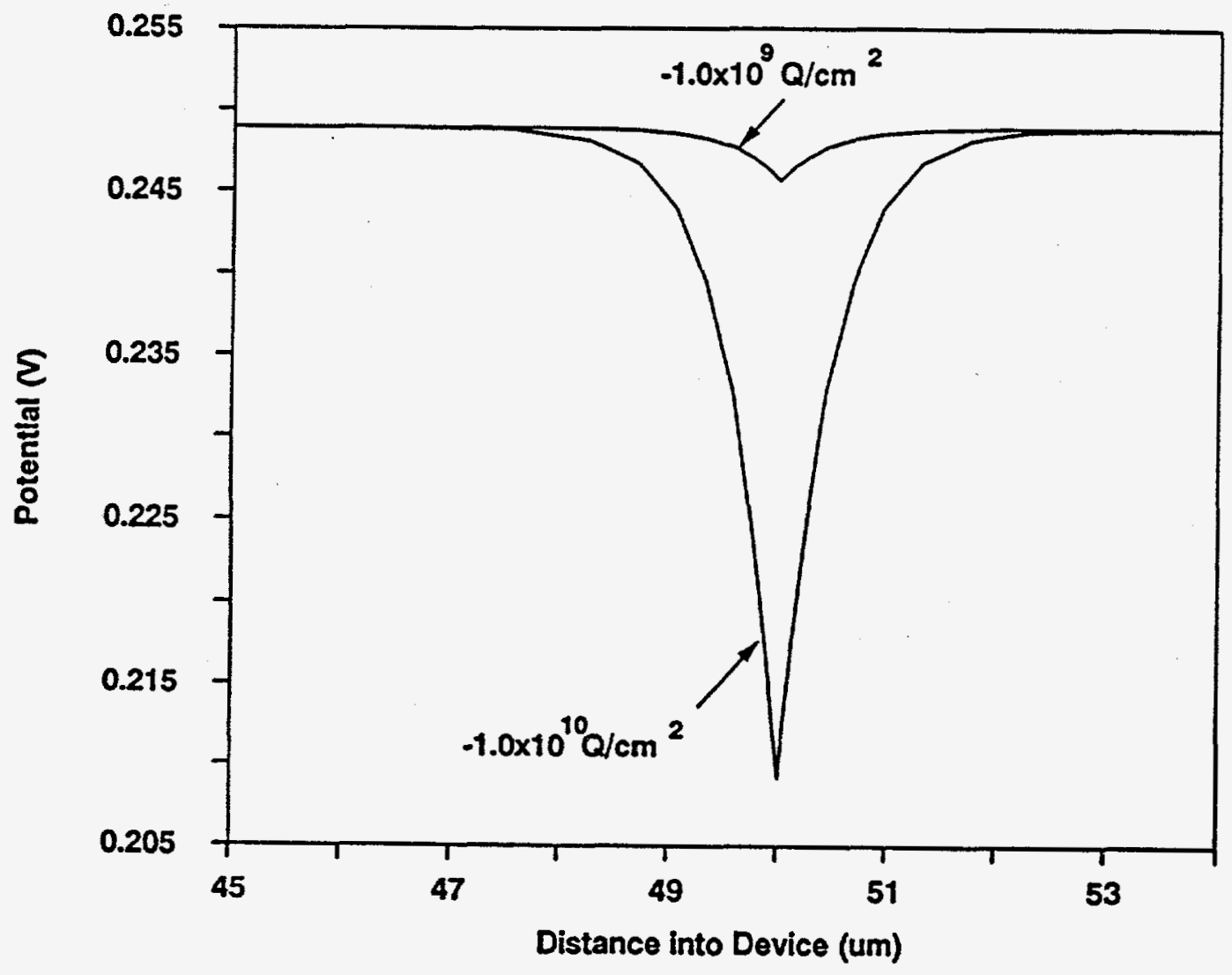

Figure 3-5. Band bending due to interface charge from hydrodynamic model. 


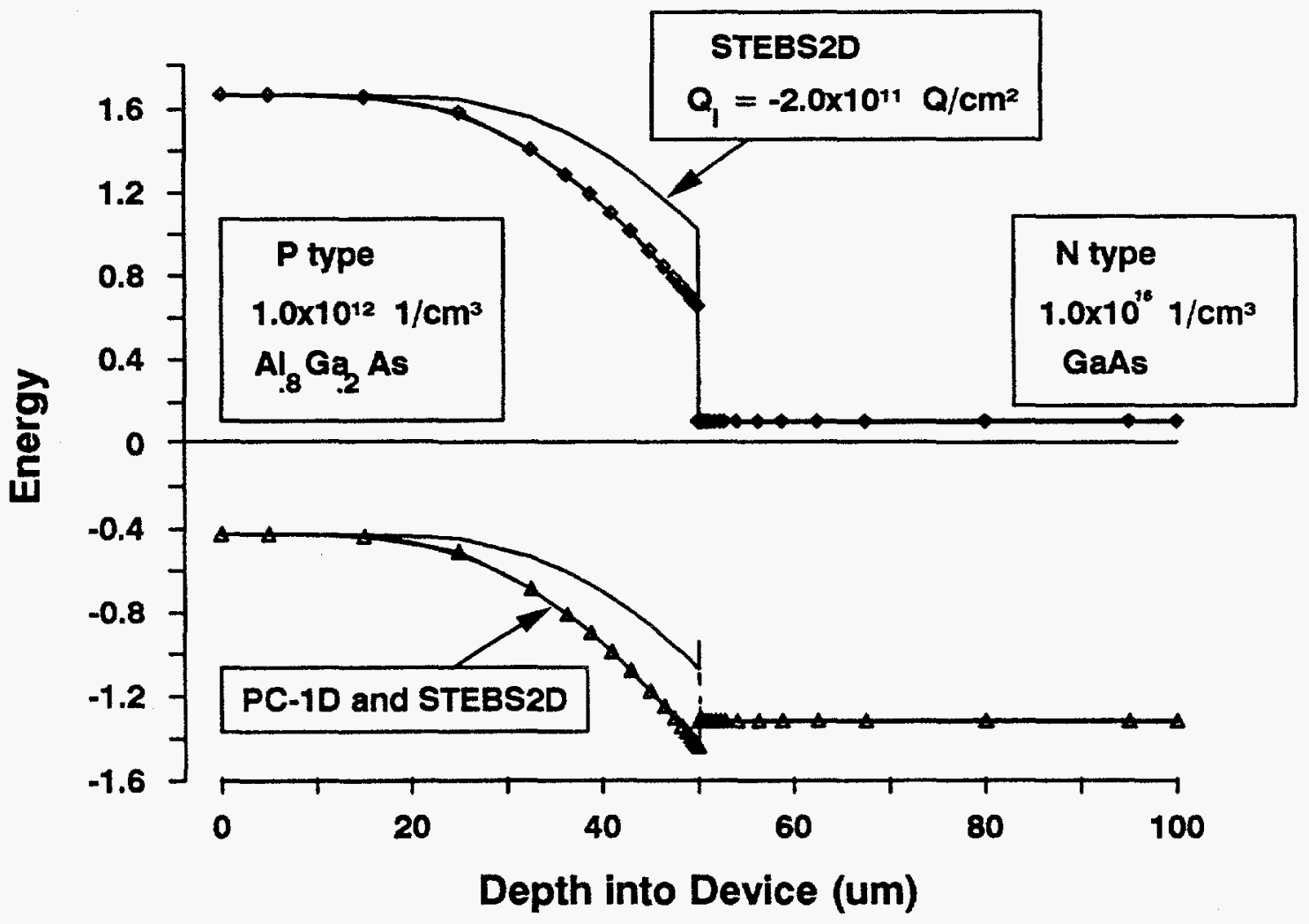

Figure 3-6. Equilibrium band diagram of an $\mathrm{Al}_{0.8} \mathrm{Ga}_{0.2} \mathrm{As} / \mathrm{GaAs}$ heterostructure, interface charge is also included in one calculation. Comparison with PC-1D is also shown. 
Verification by $P C-1 D$ is provided for the case with no interface charge located between the two materials, PC-1D does not support interface charge. 


\section{REFERENCES}

1. P. Rohr, F. Lindholm, and K. Allen, Solid-State Electronics, vol 17, pp. 729-734, (1974).

2. S.E. Laux and R.G. Byrnes, IBM J. Res. Dev., vol 29, \#3, pp. 289-301, (1985).

3. S. Selberher, Analysis and Simulation of Semiconductor Devices, Springer-Verlag, N.Y., N.Y., (1984).

4. K.K. Thornber, IEEE Elec. Dev. Lett., vol. EDL-3, \#3, pp. 69-71, (1982).

5. I.C. Kizilyalli and M. Artaki, IEEE Elec. Dev. Lett., vol. EDL-10, \#9, pp. 405-8, (1989).

6. R. Stratton, Phys. Rev., vol 126, \#6, pp. 2002-14, (1962).

7. C.C. McAndrew, E.L. Heasell, and K. Singhal, Semi. Sci. Tech., vol. 3, pp. 886-94, (1988).

8. J.P. Uyemura, Fundamentals of MOS Digital Integrated Circuits, Addison-Wesley

9. Y.J. Park, D.H. Navon, and T.W. Tang, IEEE Trans. Elec. Dev., ED-31, \#12, pp. 1724-9, (1984).

10. P.T. Nguyen, D.H. Navon, and T.W. Tang, IEEE Trans. Elec. Dev., ED-32, \#4, pp. 783-7, (1985).

11. S. Bandyopodhyay, M.E. Klausmeier-Brown, C.M. Maziar, S. Datta, and M.S. Lundstrum, IEEE Trans. Elec. Dev., ED-34, \#2, pp. 392-9, (1987).

12. W. Hänsch, S. Selberher, IEEE Trans. Elec. Dev., ED-34, \#5, pp. 1074-8, (1987).

13. R. Cook, IEEE Trans. Elec. Dev., ED-30, \#9, pp.1103-10, (1983).

14. M.S. Adler, IEEE Trans. Elec. Dev., ED-25, \#1, pp. 16-22, (1978).

15. R.S. Haung, P.H. Ladbrook, J. Appl. Phys., vol. 48, \#11, pp. 4791-8, (1977).

16. R. Cook, J. Frey, IEEE Trans. Elec. Dev., ED-29, \#6, pp.970-77, (1982).

17. M.S. Shur, L.F. Eastman, IEEE Trans. Elec. Dev., ED-26, \#11, pp. 1677-83, (1979).

18. D.J. Widiger, Ph.D. Thesis, University of Illinois at Urbana (1984).

19. K. Horio, H. Yanai, Proc. NASECODE V, J.J.H. Miller, Ed., Boole Press, Dublin, pp. 231-6, (1987). 
20. E.M. Azoff, IEEE Trans. Elec. Dev., ED-36, \#4, pp. 609-16, (1989).

21. T. Shawki, G. Salmer, and O. El-Sayed, IEEE Trans. Elec. Dev., ED-37, \#1, pp. 21-30, (1990).

22. R. Cook, J. Frey, COMPEL, vol 1, \#2, pp. 65-87, (1982).

23. W. Hänsch, M. Miura-Mattausch, J. Appl. Phys., vol. 60, \#2, pp. 650-5, (1986).

24. C.L. Wilson, IEEE Trans. Elec. Dev., ED-35, \#2, pp. 180-7, (1988).

25. A.H. Marshak, K.M. Van Vliet, Solid-State Elec., vol. 21, pp. 417-27, (1978).

26. M. Rudan and F. Odeh, COMPEL, vol 5, \#3, pp. 149-83, (1986).

27. K. Blotekaer, IEEE Trans. Elec. Dev., ED-17, \#1, pp. 38-47, (1970).

28. C.C. McAndrew, E.L. Heasell, and K. Singhal, Semicond. Sci. Tech., vol. 2, pp. 643-8, (1987).

29. E.M. Azoff, Solid-State Elec., vol. 30, \#9, pp. 913-17, (1987).

30. E.M. Azoff, J. Appl. Phys., vol 64, \#5, pp. 2439-46, (1988).

31. R. Kishore, Solid-State Elec., vol 32, \#6, pp. 469-73, (1989).

32. J.E. Sutherland and J.R. Hauser, IEEE Trans. Elec. Dev., ED-24, \#4, pp. 363-72, (1977).

33. M.S. Lundstrum, R.J. Schuelke, IEEE Trans. Elec. Dev., ED-30, \#9, pp. 1151-58, (1983).

34. A. Marshak, IEEE Trans. Elec. Dev., ED-36, \#9, pp. 1764-72, (1989).

35. S.P. Gaur, D.H. Navon, IEEE Trans. Elec. Dev., ED-23, \#1, pp. 50-7, (1976).

36. V.C. Alwin, D.H. Navon, and L.J. Turgeon, IEEE Trans. Elec. Dev., ED-24, \#11, pp. 1297-1304, (1977).

37. K. Kittel, Introduction to Solid State Physics 5th ed., J.Wiley \& Sons, N.Y., N.Y., (1976).

38. S. Wang, Solid-State Electronics, McGraw-Hill, N.Y., N.Y., (1966).

39. S. Szeto and R. Reif, Solid-State Elec., vol 32, \#4, pp. 307-15, (1989).

40. By expanding the third term of equation 32 in [30], $n v \bullet \nabla \varepsilon=n v \bullet \nabla \varepsilon_{c}-n v \bullet \nabla \chi$, equation (9) in this paper is recovered. 
41. H.H. Ou, T.W. Tang, IEEE Trans. Elec. Dev., ED-34, \#7, pp. 1533-5, (1987).

42. J.S. Blakemore, Semiconductor Statistics, N.Y., N.Y., Pergammon, (1960).

43. G. Baccarani, M.R. Wordeman, Solid-State Elec., vol. 28, \#4, pp407-16, (1985).

44. B.O. Seraphin, Solar Energy Conversion, Springer-Verlag, N.Y., N.Y., (1979).

45. S.J. Fonash, Solar Cell Device Physics, Academic Press, N.Y., N.Y., (1981).

46. P.E. Gruembaum, R.R. King, and R.M. Swanson, J. Appl. Phys., vol. 66, \#12, pp. 6110-4, (1989).

47. P.D. Taylor, Thyristor Design and Realization, John Wiley \& Sons, London, (1987).

48. R.G. Hoft, Semiconductor Power Electronics, Van Nostrand Reinhold, N.Y., N.Y., (1986).

49. B.G. Streetman, Solid State Electronic Devices, Prentice-Hall, Englewood Cliffs, N.J., (1980).

50. L.F. Eastman, Proc. IEEE/Cornell Conf. on Adv. Concepts in High Speed Semi. Dev. and Cir., pp. 1-6,(1985).

51. P.A. Basore, PC-1D Installation Manual and User's Guide Version 2.1, lowa State Univeristy Research Foundation, Ames, IA, 1989. 


\section{RE-EVALUATION OF THE DERIVATIVES OF THE HALF ORDER FERMI INTEGRALS}

The Fermi integrals of half orders are important in the simulation of semiconductor transport processes. Several of these integrals $(-1 / 2,1 / 2,3 / 2,5 / 2)$ have been recently re-tabulated since the original 1938 study [3], but the derivatives were not re-evaluated. The original integral values were calculated without the aid of high speed computers using approximate series evaluation and tabulations of exponentials and zeta functions. In addition, a discrepancy was found in the literature since the original study in 1938 . The second derivative of $F_{1 / 2}$ has been mistakenly represented as being proportional to $\mathscr{F}_{3 / 2}$ $\left(F_{-3 / 2} / \Gamma(-1 / 2)\right)$. This section tabulates the half order Fermi integrals from $-1 / 2$ to $5 / 2$ over the reduced energy range -5 to 20 in 0.25 increments. The first two derivatives of $F_{-1 / 2}$ are also calculated by numerical integration and tabulated to aid in interpolation, and it is shown that the second derivative of $F_{1 / 2}$ is not proportional to $\mathscr{F}_{3 / 2}$.

The use of Fermi-Dirac statistics is vitally important for the simulation of high injection in semiconductor devices. The integrals of various order appear in equations used for the determination of the carrier concentration, diffusion current [1], and the carrier energy flux [2]. Indeed, the recursion relation relating the derivative of a Fermi integral to one of lesser order is used in the derivation of the drift-diffusion model if FermiDirac statistics are maintained [2]. McDougall and Stoner [3] tabulated the integrals of the order $-1 / 2,1 / 2$, and $3 / 2$. The values were calculated by evaluation of series and asymptotic functions using tabulated values of the exponential and zeta functions. In addition, the recursion relation 


$$
F_{j}^{\prime}(\eta)=j F_{j-1}(\eta)
$$

for $j>0$ was proven to relate the derivative of a Fermi function to a Fermi integral of previous order. The first three derivatives of $F_{1 / 2}$ were also provided for interpolation by Taylor series.

$$
F_{\frac{1}{2}}\left(\eta_{0}+\Delta \eta\right) \approx F_{\frac{1}{2}}\left(\eta_{0}\right)+\Delta \eta F_{\frac{1}{2}}^{\prime}\left(\eta_{0}\right)+\Delta \eta^{2} F_{\frac{1}{2}}^{\prime \prime}\left(\eta_{0}\right)+\Delta \eta^{3} F_{\frac{1}{2}}^{\prime \prime \prime}\left(\eta_{0}\right)
$$

The derivatives were clearly marked in the original paper and subsequent work [4]. These tables became the basis for later work on integrals of other orders [4]. The derivative recursion relation was later re-proven by Dingle [5] using a Mellin transformation but failed to point out that its applicability was restricted to $0 \leq j \leq 1[6]$. It appears that in the widely referenced work of Blakemore [7], equation (4-1) has been extended further than intended by the original authors, because in reference [7], $\mathscr{F}_{3 / 2}$ has been listed as being proportional to $\mathrm{F}^{\prime \prime}{ }_{1 / 2}$ from reference [3]. In 1989, Cloutman [8] retabulated the Fermi integrals of order $-1 / 2,1 / 2,3 / 2$, and $5 / 2$ over the reduced energy range -5 to 25 in 0.05 step increments to an accuracy one part in $10^{10}$. The integrals were calculated using a supercomputer and tested numerical techniques. The author provides a fifth order interpolation scheme to estimate the integrals between the tabulated values. However, the first derivative of the $-1 / 2^{\text {th }}$ order integral is not calculated, making it impossible to interpolate accurately. In addition, inverse interpolation may be required which necessitates the need for derivatives of the functions.

The objective of this paper is to show that the values tabulated as $\mathscr{F}_{-3 / 2}$ in reference [7] are actually proportional to $F^{\prime \prime}{ }_{1 / 2}$, not $F_{-3 / 2}$ as intended. In addition, the 
Fermi integrals of the order $-1 / 2,1 / 2,3 / 2$, and $5 / 2$ are calculated by numerical integration and tabulated for the reduced energy range -5 to 20 in 0.25 increments. The first two derivatives of $F_{-1 / 2}$ are also tabulated over the same reduced energy range. As shown below, the derivatives were calculated by integration of the appropriate derivative functions, without using the derivative recursion relation.

\section{MATHEMATICS}

The Fermi function of order $j$ is an improper integral of the form

$$
F_{j}(\eta)=\int_{0}^{\infty} \frac{e^{j}}{(1+\exp (\varepsilon-\eta))} d \varepsilon .
$$

where in the case of semiconductor analysis.

$$
\begin{aligned}
& \varepsilon=\left[\frac{\left(E-E_{c}\right)}{K T}\right] \\
& \eta=\left[\frac{\left(E_{f}-E_{c}\right)}{K T}\right]
\end{aligned}
$$

Normally in semiconductor physics a related function of the form

$$
\mathscr{F}_{j}(\eta)=\frac{1}{\Gamma(j+1)} \int_{0}^{\infty} \frac{e^{j}}{(1+\exp (e-\eta))} d \varepsilon
$$

is used in the analysis. The gamma function serves to normalize the probability density to one. Since the gamma function is well behaved and tabulated for all argument values except negative integers, there is no disadvantage in working with equation (4-3) over 
equation (4-4). The final result of the integration of equation (4-3) may be divided by the appropriate gamma function to obtain the desired value.

To include Fermi-Dirac statistics in a device simulation employing numerical techniques such as the finite element or finite difference, it is more convenient and less computationaly intensive to use tabulated values and interpolation rather than evaluating the integral itself. A simple choice is the Taylor series expansion like equation (4-2) which requires the values of the first three derivatives at the evaluated points. Unfortunately, this interpolation does not provide a satisfactory error term. An interpolation scheme which does provide sufficient accuracy would be a fifth order Hermite method. One fifth order method was proposed by Cloutman [8] which requires the functional values and its first derivative at three points. The method proposed in this paper uses two points and values of the function and the first two derivatives at those points for the interpolation. This latter approach will provide smoother interpolation near the tabulated values. In most interpolation schemes the derivatives of the functions are required, therefore, the applicability and origins of equation (4-1) are re-examined.

$$
F_{j}^{\prime}(\eta)=\frac{\partial}{\partial \eta} F_{j}(\eta)=\int_{0}^{\infty} \frac{\partial}{\partial \eta} \frac{e^{j}}{(1+\exp (\varepsilon-\eta))} d \varepsilon=\int_{0}^{\infty} \frac{e^{\prime} \exp (\varepsilon-\eta)}{(1+\exp (\varepsilon-\eta))^{2}} d \varepsilon
$$

Note that the last integral may be rewritten as

$$
\frac{\partial}{\partial \eta} F_{j}(\eta)=-\int_{0}^{\infty} \epsilon^{j} \frac{\partial}{\partial \varepsilon} \frac{1}{(1+\exp (\varepsilon-\eta))} d \varepsilon
$$


Now integration by parts results in the following form for the derivative

$$
\frac{\partial}{\partial \eta} F_{j}(\eta)=\left.\frac{e^{j}}{(1+\exp (\varepsilon-\eta))}\right|_{0} ^{\infty}+j \int_{0}^{\infty} \frac{e^{j-1}}{(1+\exp (e-\eta))} d e
$$

Clearly the second term is proportional to a fermi integral of one less order. However, if $j$ is less than zero a singularity develops in the first term, not allowing its evaluation at $\varepsilon=0$. Therefore, McDougall and Stoner were correct in limiting the range of equation (4-1) to $j \geq 0$, which leads to the conclusion $F_{-1 / 2}^{\prime} \neq-(1 / 2) F_{-3 / 2}$. Since the recursion relationship breaks down for $j<0$, the values of the first derivative may still be evaluated from

$$
\frac{\partial}{\partial \eta} F_{j}(\eta)=\int_{0}^{\infty} \frac{e^{\prime} \exp (e-\eta)}{(1+\exp (e-\eta))^{2}} d e
$$

for $\mathrm{j}<0$. The values of the second derivative may be found by evaluating

$$
F_{f}^{\prime \prime}(\eta)=\int_{0}^{\infty} \frac{\epsilon^{j} \exp (\epsilon-\eta)[\exp (\epsilon-\eta)-1]}{(1+\exp (\varepsilon-\eta))^{3}} d \epsilon
$$

which is obtained by differentiating equation (4-8) with respect to $\eta$. These last two integrals are general forms but need be applied only when $\mathrm{j}<0$. The tabulated values of the functions and the derivatives, in conjunction with the following proposed fifth order interpolation scheme, should provide interpolation to an accuracy of 1 part in $10^{10}$ for all orders of the Fermi integrals discussed in this section $(-1 / 2,1 / 2,3 / 2$, and $5 / 2)$. 


$$
\begin{aligned}
& f(x)=f(a)\left[1-\frac{(x-a)^{3}}{(b-a)^{3}}+\frac{3(x-a)^{3}(b-x)}{(b-a)^{4}}-\frac{6(x-a)^{3}(b-x)^{2}}{(b-a)^{5}}\right] \\
& +f(b)\left[\frac{(x-a)^{3}}{(b-a)^{3}}-\frac{3(x-a)^{3}(b-x)}{(b-a)^{4}}+\frac{6(x-a)^{3}(b-x)^{2}}{(b-a)^{5}}\right] \\
& +f^{\prime}(a)\left[(x-a)-\frac{(x-a)^{3}}{(b-a)^{2}}+\frac{2(x-a)^{3}(b-x)}{(b-a)^{3}}-\frac{3(x-a)^{3}(b-x)^{2}}{(b-a)^{4}}\right] \\
& +f^{\prime}(b)\left[\frac{(x-a)^{3}(b-x)}{(b-a)^{3}}-\frac{3(x-a)^{3}(b-x)^{2}}{(b-a)^{4}}\right] \\
& +\frac{f^{\prime \prime}(a)}{2}\left[(x-a)^{2}-\frac{(x-a)^{3}}{(b-a)}+\frac{(x-a)^{3}(b-x)}{(b-a)^{2}}-\frac{(x-a)^{3}(b-x)^{2}}{(b-a)^{3}}\right] \\
& +\frac{f^{\prime \prime}(b)}{2}\left[\frac{(x-a)^{3}(b-x)^{2}}{(b-a)^{3}}\right]
\end{aligned}
$$

In this proposed scheme the interpolation at point $\mathrm{x}$ is bounded by the tabulated values of the integrals at $a$ and $b$, and the integrals used in the interpolation of the different orders are given in Table 4-1.

\section{EVALUATION OF INTEGRALS}

The integrals listed in equations (4-3), (4-8), and (4-9) were evaluated using the Romberg integration routines on open intervals [9]. First, the interval of integration $(0$ to $\infty)$ is split into two intervals, $(0$ to 10$)$ and $(10$ to $\infty$. The lower limit on the first interval produces a singularity when the exponent of $e^{j}$ becomes less than zero. To alleviate this problem, all of the integrals for the first interval were transformed using the relationship 


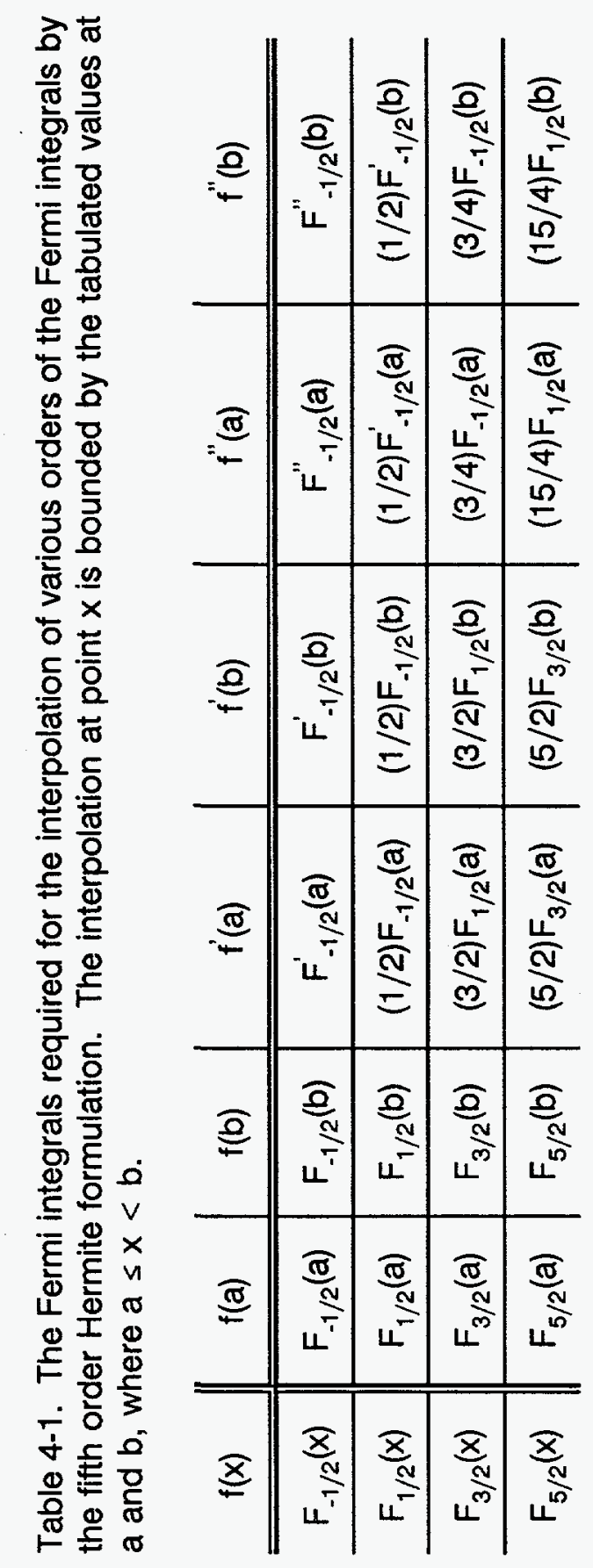




$$
\int_{a}^{b} f(x) d x=\int_{0}^{\sqrt{b-a}} 2 t f\left(t^{2}+a\right) d t \quad \text { for }(b>a)
$$

where:

$$
\begin{aligned}
& x=t^{2} \\
& a=0 \\
& b=10
\end{aligned}
$$

This transformation is the same as that used by Cloutman [8]. The first interval is then integrated using an extended midpoint rule, in which a variable step size is employed. The precession was set to $1.0 \times 10^{-9}$, and the step size was tripled until this criteria was achieved. The maximum number of steps was set to $3^{8}$. For the second interval the transformation

$$
\int_{a}^{b} f(x) d x=\int_{\frac{1}{b}}^{\frac{1}{a}} \frac{1}{t^{2}} f\left(\frac{1}{t}\right) d t \quad \text { for } a>0 ; b>0
$$

where:

$$
\begin{aligned}
& x=\frac{1}{t} \\
& a=10 \\
& b=\infty
\end{aligned}
$$

was used to map the infinite range to a finite space. The same Romberg integration routine with the same precession and maximum number of steps was employed. The results of the two integrations were then added to obtain the final answer. This integration routine was placed within a loop to calculate all of the half order Fermi functions over the range of $\eta$ values desired by solving equation (4-3). Then the program 
was modified so the function being integrated was equation (4-8) to calculate the derivatives. This provided another consistency check on the values from the integration of equation (4-3), in that for $j>0$ the results of the integration of equation (8) were proportional to the next higher fermi integral from the integration of equation $(3)\left(F_{j}^{\prime}(\eta)=\right.$ $\left.j F_{j-1}(\eta)\right)$. The second derivative formula (equation (4-9)), was also evaluated for all of the Fermi integrals considered to check the results of the calculation. In this case, the result of one calculation was proportional to the Fermi integral two orders higher calculated in the initial evaluation of the Fermi function (equation (4-3)).

\section{RESULTS}

Table 4-2 contains the results of the integrations for the half order Fermi integrals; for $\mathrm{j}>-1 / 2$ the answers closely match those given in references [3] and [8]. The first two columns in Table 2 contain the values of the second and first derivative of $F_{-1 / 2}$ over the same reduced energy range. As stated previously, these values were calculated by numerical integration. Not all of the derivatives of the other orders are listed since this would replicate a large portion of the table and were simply used to check the calculation of the previous order. Notice that the sign of the second derivative of $F_{-1 / 2}$ changes. This is the first indication that this derivative is not related to $F_{-3 / 2}$. Upon examination of the basic form of the fermi integral (equation (4-1)) it is evident that the integral may not change sign as the reduced energy changes from -5 to 20 . A stronger case for the conclusion that the first derivative of $F_{-1 / 2}$ is not proportional to $F_{-3 / 2}$ will now be given. First it should be observed that the integral corresponding to $F_{-3 / 2}$ diverges as the lower limit of integration approaches zero from the left hand side. Therefore, the lower limit of the integration was set to $1.0 \times 10^{-10}$. This should be small enough to show that the 
integral is very large but large enough to avoid the singularity at zero. The results of the integration $\left(\sim F_{-3 / 2}(\eta)\right)$ are compared to the values of the first derivative of $F_{-1 / 2}$ (from Table 4-2) and $\mathscr{F}_{-3 / 2}$ in Table 4-3. The values listed under $\mathscr{I}_{-3 / 2}$ in Table 4-3 were taken from reference [7] for selected values of reduced energy. The ratios of $F_{-3 / 2} / F_{-1 / 2}$ and $F_{-3 / 2} / F_{-}$ $3 / 2$ are listed in the fourth and fifth columns. Clearly, the calculated values of $F_{-3 / 2}$ are not proportional to the calculated values $F_{-1 / 2}^{\prime}$ or $\mathscr{F}_{-3 / 2}$ from reference [7] because the ratios in columns 4 and 5 of Table $4-3$ are not constant. This implies; (i) $F_{-1 / 2} \neq-F_{-3 / 2} / 2$ and (ii) $F_{-3 / 2}$ calculated in this section is different from $\mathscr{F}_{-3 / 2}$ in reference [7]. Furthermore, examination of column 6 in Table 4-3, shows that the ratio of $F_{-1 / 2}^{\prime} / \mathscr{F}_{-3 / 2}$ is constant and equal to $\pi^{1 / 2} ; F_{-1 / 2}^{\prime}$ calculated here matches closely the values tabulated in reference [3]. This leads the conclusion that $\mathscr{F}_{-3 / 2}$ in reference [7] is in no way related to $F_{-3 / 2}$, but is related to $\mathrm{F}_{-1 / 2}$

\section{CONCLUSIONS}

The half order fermi integrals from $-1 / 2$ to $5 / 2$ have been computed over the reduced energy range -5 to 20 in 0.25 increments. In addition, the first and second derivatives of the $-1 / 2$ order integrals have been tabulated over the same energy range. Using these values of the derivatives with the fifth order Hermite scheme provide the capability of interpolating the half order integrals from $-1 / 2$ to $5 / 2$ to 1 part in $10^{10}$. It is shown that the inconsistency between the tabulated values of $F_{-3 / 2}$ in this paper and $\mathfrak{Z}_{-3 / 2}$ in reference [7] is due to the incorrect assumption that $F^{\prime \prime}{ }_{1 / 2}$ is proportional to $F_{-3 / 2}$ in reference[7]. 
Tabel 4-2. Values from the integration of equations (3), (8), and (9) for the reduced energy range -5 to 20 in 0.25 intervals.

\begin{tabular}{|c|c|c|c|c|c|c|}
\hline$\eta$ & $F_{-1 / 2}^{\prime}$ & $F_{-1 / 2}^{\prime}$ & $F_{-1 / 2}$ & $F_{1 / 2}$ & $F_{3 / 2}$ & $F_{5 / 2}$ \\
\hline-5 & $1.1717886992 \mathrm{e}-02$ & $1.1829831219 \mathrm{e}-02$ & $1.1886110956 \mathrm{e}-02$ & $5.9571768454 \mathrm{e}-03$ & $8.9463822604 \mathrm{e}-03$ & $2.2379248359 \mathrm{e}-02$ \\
\hline-4.75 & $1.4965364858 \mathrm{e}-02$ & $1.5149073196 \mathrm{e}-02$ & $1.5241575399 \mathrm{e}-02$ & $7.6440217846 \mathrm{e}-03$ & $1.1483512943 \mathrm{e}-02$ & $2.8730678137 \mathrm{e}-02$ \\
\hline-4.5 & $1.9083912463 \mathrm{e}-02$ & $1.9384996673 \mathrm{e}-02$ & $1.9536902017 \mathrm{e}-02$ & $9.8066560914 \mathrm{e}-03$ & $1.4738753194 \mathrm{e}-02$ & $3.6882940385 \mathrm{e}-02$ \\
\hline-4.25 & $2.4288811805 \mathrm{e}-02$ & $2.4781442353 \mathrm{e}-02$ & $2.5030620331 \mathrm{e}-02$ & $1.2578086067 \mathrm{e}-02$ & $1.8914453703 e-02$ & $4.7345492870 \mathrm{e}-02$ \\
\hline-4 & $3.0836896403 \mathrm{e}-02$ & $3.1641216235 \mathrm{e}-02$ & $3.2049374030 \mathrm{e}-02$ & $1.6127737810 \mathrm{e}-02$ & $2.4269405335 \mathrm{e}-02$ & $6.0771189375 \mathrm{e}-02$ \\
\hline-3.75 & $3.9026962393 \mathrm{e}-02$ & $4.0336617782 \mathrm{e}-02$ & $4.1003975928 \mathrm{e}-02$ & $2.0670947458 \mathrm{e}-02$ & $3.1134213256 \mathrm{e}-02$ & $7.7996173966 \mathrm{e}-02$ \\
\hline-3.5 & $4.9194266975 \mathrm{e}-02$ & $5.1319404321 \mathrm{e}-02$ & $5.2408055172 \mathrm{e}-02$ & $2.6480621748 \mathrm{e}-02$ & $3.9930626695 \mathrm{e}-02$ & $1.0009057784 \mathrm{e}-01$ \\
\hline-3.25 & $6.1694859455 \mathrm{e}-02$ & $6.5128214650 \mathrm{e}-02$ & $6.6898930749 \mathrm{e}-02$ & $3.3901375181 \mathrm{e}-02$ & $5.1195697034 \mathrm{e}-02$ & $1.2842275774 \mathrm{e}-01$ \\
\hline-3 & $7.6873823005 \mathrm{e}-02$ & $8.2390205158 \mathrm{e}-02$ & $8.5259701126 \mathrm{e}-02$ & $4.3366367386 \mathrm{e}-02$ & $6.5611738806 \mathrm{e}-02$ & $1.6474039373 \mathrm{e}-01$ \\
\hline-2.75 & $9.5010014363 e-02$ & $1.0381197364 \mathrm{e}-01$ & $1.0844052624 \mathrm{e}-01$ & $5.5416879018 \mathrm{e}-02$ & $8.4043121242 \mathrm{e}-02$ & $2.1127239367 \mathrm{e}-01$ \\
\hline-2.5 & $1.1622956489 \mathrm{e}-01$ & $1.3015291246 \mathrm{e}-01$ & $1.3757560702 \mathrm{e}-01$ & $7.0724324684 \mathrm{e}-02$ & $1.0758087439 \mathrm{e}-01$ & $2.7085618653 \mathrm{e}-01$ \\
\hline-2.25 & $1.4038308662 \mathrm{e}-01$ & $1.6217244560 \mathrm{e}-01$ & $1.7399042841 \mathrm{e}-01$ & $9.0113847767 \mathrm{e}-02$ & $1.3759588460 \mathrm{e}-01$ & $3.4709554766 \mathrm{e}-01$ \\
\hline-2 & $1.6689000183 \mathrm{e}-01$ & $2.0054224555 \mathrm{e}-01$ & $2.1919160690 \mathrm{e}-01$ & $1.1458782471 \mathrm{e}-01$ & $1.7580098885 \mathrm{e}-01$ & $4.4455445346 \mathrm{e}-01$ \\
\hline-1.75 & $1.9457057311 \mathrm{e}-01$ & $2.4571732049 \mathrm{e}-01$ & $2.7482971737 \mathrm{e}-01$ & $1.4534651734 \mathrm{e}-01$ & $2.2432145429 \mathrm{e}-01$ & $5.6899242074 \mathrm{e}-01$ \\
\hline-1.5 & $2.2151294030 \mathrm{e}-01$ & $2.9776817460 \mathrm{e}-01$ & $3.4262484652 \mathrm{e}-01$ & $1.8380185764 \mathrm{e}-01$ & $2.8577205976 \mathrm{e}-01$ & $7.2764609262 \mathrm{e}-01$ \\
\hline-1.25 & $2.4505277333 \mathrm{e}-01$ & $3.5619191404 \mathrm{e}-01$ & $4.2424696959 \mathrm{e}-01$ & $2.3157914756 \mathrm{e}-01$ & $3.6333725792 \mathrm{e}-01$ & $9.2956019882 \mathrm{e}-01$ \\
\hline-1 & $2.6195978830 \mathrm{e}-01$ & $4.1974220685 \mathrm{e}-01$ & $5.2115038100 \mathrm{e}-01$ & $2.9050089617 \mathrm{e}-01$ & $4.6084880629 \mathrm{e}-01$ & $1.1859681755 \mathrm{e}+00$ \\
\hline
\end{tabular}




\begin{tabular}{|c|c|c|c|c|c|c|}
\hline$\eta$ & $F_{-1 / 2}^{\prime \prime}$ & $F_{-1 / 2}^{\prime}$ & $F_{-1 / 2}$ & $F_{1 / 2}$ & $\mathrm{~F}_{3 / 2}$ & $F_{5 / 2}$ \\
\hline-0.75 & $2.6889994856 \mathrm{e}-01$ & $4.8633957615 \mathrm{e}-01$ & $6.3437421299 \mathrm{e}-01$ & $3.6254762788 \mathrm{e}-01$ & $5.8285315247 \mathrm{e}-01$ & $1.5107185530 \mathrm{e}+00$ \\
\hline-0.5 & $2.6315095379 \mathrm{e}-01$ & $5.5313076318 \mathrm{e}-01$ & $7.6433782844 \mathrm{e}-01$ & $4.4979304683 \mathrm{e}-01$ & $7.3465933286 \mathrm{e}-01$ & $1.9207378736 \mathrm{e}+00$ \\
\hline-0.25 & $2.4340376067 \mathrm{e}-01$ & $6.1674278446 \mathrm{e}-01$ & $9.1067493261 \mathrm{e}-01$ & $5.5431553554 \mathrm{e}-01$ & $9.2235798483 \mathrm{e}-01$ & $2.4365149793 \mathrm{e}+00$ \\
\hline 0 & $2.1035636633 \mathrm{e}-01$ & $6.7371823886 \mathrm{e}-01$ & $1.0721549336 e+00$ & $6.7809389515 \mathrm{e}-01$ & $1.1528038371 \mathrm{e}+00$ & $3.0825860829 e+00$ \\
\hline 0.25 & $1.6680553553 \mathrm{e}-01$ & $7.2104114923 \mathrm{e}-01$ & $1.2467270783 \mathrm{e}+00$ & $8.2290069075 \mathrm{e}-01$ & $1.4335582219 \mathrm{e}+00$ & $3.8879964505 \mathrm{e}+00$ \\
\hline 0.5 & $1.1712165923 \mathrm{e}-01$ & $7.5660823328 \mathrm{e}-01$ & $1.4316924543 e+00$ & $9.9020924871 \mathrm{e}-01$ & $1.7727936119 e+00$ & $4.8867140153 e+00$ \\
\hline 0.75 & $6.6272768628 \mathrm{e}-02$ & $7.7950627437 \mathrm{e}-01$ & $1.6239720046 \mathrm{e}+00$ & $1.1811286608 \mathrm{e}+00$ & $2.1791681706 \mathrm{e}+\infty 0$ & $6.1179734104 e+00$ \\
\hline 1 & $1.8768151911 \mathrm{e}-02$ & $7.9002688672 \mathrm{e}-01$ & $1.8204113571 \mathrm{e}+00$ & $1.3963752868 \mathrm{e}+00$ & $2.6616826248 \mathrm{e}+00$ & $7.6265353551 e+00$ \\
\hline 1.25 & $-2.2111166135 \mathrm{e}-02$ & $7.8944684719 e-01$ & $2.0180586384 \mathrm{e}+00$ & $1.6362812505 \mathrm{e}+00$ & $3.2295334982 \mathrm{e}+00$ & $9.4628547237 \mathrm{e}+00$ \\
\hline 1.5 & $-5.4612714808 \mathrm{e}-02$ & $7.7967342641 \mathrm{e}-01$ & $2.2143679789 \mathrm{e}+00$ & $1.9008334611 \mathrm{e}+00$ & $3.8919755410 \mathrm{e}+00$ & $1.1683159163 e+01$ \\
\hline 1.75 & $-7.8385132412 \mathrm{e}-02$ & $7.6287077918 \mathrm{e}-01$ & $2.4073097678 \mathrm{e}+00$ & $2.1897321692 \mathrm{e}+00$ & $4.6582028212 e+00$ & $1.4349447177 \mathrm{e}+01$ \\
\hline 2 & $-9.4099220608 \mathrm{e}-02$ & $7.4115397721 \mathrm{e}-01$ & $2.5953945742 \mathrm{e}+00$ & $2.5024578260 \mathrm{e}+00$ & $5.5372536751 \mathrm{e}+00$ & $1.7529419173 e+01$ \\
\hline 2.25 & $-1.0300745896 \mathrm{e}-01$ & $7.1638885833 \mathrm{e}-01$ & $2.7776337057 e+00$ & $2.8383366526 \mathrm{e}+00$ & $6.5379407322 e+00$ & $2.1296357054 e+01$ \\
\hline 2.5 & $-1.0657014848 \mathrm{e}-01$ & $6.9009553850 \mathrm{e}-01$ & $2.9534626871 \mathrm{e}+00$ & $3.1965986994 \mathrm{e}+00$ & $7.6688042644 \mathrm{e}+00$ & $2.5728967292 e+01$ \\
\hline 2.75 & $-1.0619999767 \mathrm{e}-01$ & $6.6343097122 \mathrm{e}-01$ & $3.1226514661 \mathrm{e}+00$ & $3.5764253086 \mathrm{e}+00$ & $8.9380853738 \mathrm{e}+00$ & $3.0911201543 e+01$ \\
\hline 3 & $-1.0312229641 \mathrm{e}-01$ & $6.3722048447 \mathrm{e}-01$ & $3.2852167829 \mathrm{e}+00$ & $3.9769853541 \mathrm{e}+00$ & $1.0353714865 \mathrm{e}+01$ & $3.6932065412 e+01$ \\
\hline 3.25 & $-9.8322304691 \mathrm{e}-02$ & $6.1201267700 \mathrm{e}-01$ & $3.4413458636 \mathrm{e}+00$ & $4.3974611790 \mathrm{e}+00$ & $1.1923313730 \mathrm{e}+01$ & $4.3885424173 e+01$ \\
\hline 3.5 & $-9.2547146850 \mathrm{e}-02$ & $5.8814001423 \mathrm{e}-01$ & $3.5913348247 \mathrm{e}+00$ & $4.8370658976 \mathrm{e}+00$ & $1.3654201686 e+01$ & $5.1869811468 \mathrm{e}+01$ \\
\hline 3.75 & $-8.6335567200 \mathrm{e}-02$ & $5.6577500397 \mathrm{e}-01$ & $3.7355418182 \mathrm{e}+00$ & $5.2950539324 \mathrm{e}+00$ & $1.5553410871 e+01$ & $6.0988245087 e+01$ \\
\hline
\end{tabular}




\begin{tabular}{|c|c|c|c|c|c|c|}
\hline$\eta$ & $F_{-1 / 2}^{\prime}$ & $\mathrm{F}_{-1 / 2}^{\prime}$ & $F_{-1 / 2}$ & $F_{1 / 2}$ & $\mathrm{~F}_{3 / 2}$ & $F_{5 / 2}$ \\
\hline 4 & $-8.0057672473 \mathrm{e}-02$ & $5.4497740003 \mathrm{e}-01$ & $3.8743531182 \mathrm{e}+00$ & $5.7707265277 \mathrm{e}+00$ & $1.7627702501 \mathrm{e}+01$ & $7.1348052324 \mathrm{e}+01$ \\
\hline 4.25 & $-7.3954439680 \mathrm{e}-02$ & $5.2573135181 \mathrm{e}-01$ & $4.0081599464 e+00$ & $6.2634337132 e+00$ & $1.9883584889 \mathrm{e}+01$ & $8.3060706248 \mathrm{e}+01$ \\
\hline 4.5 & $-6.8172151592 \mathrm{e}-02$ & $5.0797325114 \mathrm{e}-01$ & $4.1373428972 \mathrm{e}+00$ & $6.7725738818 \mathrm{e}+00$ & $2.2327331715 e+01$ & $9.6241673357 e+01$ \\
\hline 4.75 & $-6.2790188122 \mathrm{e}-02$ & $4.9161179080 \mathrm{e}-01$ & $4.2622629936 \mathrm{e}+00$ & $7.2975918531 e+00$ & $2.4964999841 \mathrm{e}+01$ & $1.1101027254 \mathrm{e}+02$ \\
\hline 5 & $-5.7842325155 \mathrm{e}-02$ & $4.7654190047 \mathrm{e}-01$ & $4.3832564346 \mathrm{e}+00$ & $7.8379760568 \mathrm{e}+00$ & $2.7802446213 e+01$ & $1.2748954490 e+02$ \\
\hline 5.25 & $-5.3332444748 \mathrm{e}-02$ & $4.6265407897 \mathrm{e}-01$ & $4.50063244445 \mathrm{e}+00$ & $8.3932552731 \mathrm{e}+\infty 0$ & $3.0845343607 e+01$ & $1.4580613379 \mathrm{e}+02$ \\
\hline 5.5 & $-4.9245770668 \mathrm{e}-02$ & $4.4984038162 \mathrm{e}-01$ & $4.6146729696 \mathrm{e}+00$ & $8.9629952264 e+00$ & $3.4099195125 \mathrm{e}+01$ & $1.6609017431 e+02$ \\
\hline 5.75 & $-4.55566687119 e-02$ & $4.3799804628 \mathrm{e}-01$ & $4.7256335618 \mathrm{e}+00$ & $9.5467952206 \mathrm{e}+00$ & $3.7569347414 \mathrm{e}+01$ & $1.8847519140 \mathrm{e}+02$ \\
\hline 6 & $-4.2234035955 \mathrm{e}-02$ & $4.2703149673 \mathrm{e}-01$ & $4.8337449521 \mathrm{e}+00$ & $1.0144284933 e+01$ & $4.1261002648 \mathrm{e}+01$ & $2.1309800596 \mathrm{e}+02$ \\
\hline 6.25 & $-3.9244601494 \mathrm{e}-02$ & $4.1685326096 \mathrm{e}-01$ & $4.9392149797 \mathrm{e}+00$ & $1.0755121431 \mathrm{e}+01$ & $4.5179229358 \mathrm{e}+01$ & $2.4009864791 e+02$ \\
\hline 6.5 & $-3.6555320891 \mathrm{e}-02$ & $4.0738418747 \mathrm{e}-01$ & $5.0422306568 \mathrm{e}+00$ & $1.1378986439 \mathrm{e}+01$ & $4.9328972181 \mathrm{e}+01$ & $2.6962027587 e+02$ \\
\hline 6.75 & $-3.4134615766 \mathrm{e}-02$ & $3.9855322553 \mathrm{e}-01$ & $5.1429602899 \mathrm{e}+00$ & $1.2015583862 e+01$ & $5.3715060638 \mathrm{e}+01$ & $3.0180910244 e+02$ \\
\hline 7 & $-3.1953129781 \mathrm{e}-02$ & $3.9029695187 \mathrm{e}-01$ & $5.2415551408 \mathrm{e}+00$ & $1.2664637569 e+01$ & $5.8342217025 \mathrm{e}+01$ & $3.3681432481 e+02$ \\
\hline 7.25 & $-2.9984074574 \mathrm{e}-02$ & $3.8255896559 \mathrm{e}-01$ & $5.3381518771 \mathrm{e}+00$ & $1.3325889407 e+01$ & $6.3215063517 \mathrm{e}+01$ & $3.7478805992 e+02$ \\
\hline 7.5 & $-2.8203318331 \mathrm{e}-02$ & $3.7528923056 \mathrm{e}-01$ & $5.4328736287 \mathrm{e}+00$ & $1.3999097432 \mathrm{e}+0 \mathrm{1}^{2}$ & $6.8338128575 \mathrm{e}+01$ & $4.1588528401 \mathrm{e}+02$ \\
\hline 7.75 & $-2.6589333449 \mathrm{e}-02$ & $3.6844341459 \mathrm{e}-01$ & $5.5258318049 \mathrm{e}+00$ & $1.4684034349 \mathrm{e}+01$ & $7.3715852697 \mathrm{e}+01$ & $4.6026377555 \mathrm{e}+02$ \\
\hline 8 & $-2.5123036642 \mathrm{e}-02$ & $3.6198226276 \mathrm{e}-01$ & $5.6171273784 e+00$ & $1.5380486128 \mathrm{e}+01$ & $7.9352593682 \mathrm{e}+01$ & $5.0808406240 \mathrm{e}+02$ \\
\hline 8.25 & $-2.3787597012 e-02$ & $3.5587099827 \mathrm{e}-01$ & $5.7068520815 \mathrm{e}+00$ & $1.6088250758 \mathrm{e}+01$ & $8.5252631354 \mathrm{e}+01$ & $5.5950937142 e+02$ \\
\hline 8.5 & $-2.2568220108 \mathrm{e}-02$ & $3.5007879741 \mathrm{e}-01$ & $5.7950894574 \mathrm{e}+00$ & $1.6807137184 \mathrm{e}+01$ & $9.1420171885 e+01$ & $6.1470558125 \mathrm{e}+02$ \\
\hline
\end{tabular}




\begin{tabular}{|c|c|c|c|c|c|c|}
\hline$\eta$ & $F^{\prime \prime 1 / 2}$ & $F_{-1 / 2}^{\prime}$ & $F_{-1 / 2}$ & $\mathrm{~F}_{1 / 2}$ & $\mathrm{~F}_{3 / 2}$ & $\mathrm{~F}_{5 / 2}$ \\
\hline 8.75 & $-2.1451931086 \mathrm{e}-02$ & $3.4457830203 e-01$ & $5.8819157818 \mathrm{e}+00$ & $1.7536964330 \mathrm{e}+01$ & $9.7859351712 e+01$ & $6.7384117750 e+02$ \\
\hline 9 & $-2.0427365757 \mathrm{e}-02$ & $3.3934519215 \mathrm{e}-01$ & $5.9674008832 \mathrm{e}+00$ & $1.8277560244 e+01$ & $1.0457424111 \mathrm{e}+02$ & $7.3708721024 \mathrm{e}+02$ \\
\hline 9.25 & $-1.9484575161 \mathrm{e}-02$ & $3.3435780802 \mathrm{e}-01$ & $6.0516095877 \mathrm{e}+00$ & $1.9028761337 \mathrm{e}+01$ & $1.1156884747 \mathrm{e}+02$ & $8.0461725359 \mathrm{e}+02$ \\
\hline 9.5 & $-1.8614847127 e-02$ & $3.2959681901 \mathrm{e}-01$ & $6.1345986478 \mathrm{e}+00$ & $1.9790411704 \mathrm{e}+01$ & $1.1884711827 \mathrm{e}+02$ & $8.7660736725 \mathrm{e}+02$ \\
\hline 9.75 & $-1.7810546900 \mathrm{e}-02$ & $3.2504493474 \mathrm{e}-01$ & $6.2164246785 \mathrm{e}+00$ & $2.0562362519 \mathrm{e}+01$ & $1.2641294385 \mathrm{e}+02$ & $9.5323605996 \mathrm{e}+02$ \\
\hline 10 & $-1.7064977525 \mathrm{e}-02$ & $3.2068665358 \mathrm{e}-01$ & $6.2971372444 \mathrm{e}+00$ & $2.1344471493 e+01$ & $1.3427015999 \mathrm{e}+02$ & $1.0346842547 \mathrm{e}+03$ \\
\hline 10.25 & $-1.6372258461 \mathrm{e}-02$ & $3.1650804375 \mathrm{e}-01$ & $6.3767829741 \mathrm{e}+00$ & $2.2136602391 \mathrm{e}+01$ & $1.4242255026 \mathrm{e}+02$ & $1.1211352554 \mathrm{e}+03$ \\
\hline 10.5 & $-1.5727219268 \mathrm{e}-02$ & $3.1249655274 \mathrm{e}-01$ & $6.4554051895 \mathrm{e}+00$ & $2.2938624598 \mathrm{e}+01$ & $1.5087384820 \mathrm{e}+02$ & $1.2127747250 \mathrm{e}+03$ \\
\hline 10.75 & $-1.5125305734 \mathrm{e}-02$ & $3.0864084179 \mathrm{e}-01$ & $6.5330442292 \mathrm{e}+00$ & $2.3750412727 \mathrm{e}+01$ & $1.5962774020 \mathrm{e}+02$ & $1.3097906059 \mathrm{e}+03$ \\
\hline 11 & $-1.4562497561 \mathrm{e}-02$ & $3.0493064237 \mathrm{e}-01$ & $6.6097377337 \mathrm{e}+00$ & $2.4571846329 \mathrm{e}+01$ & $1.6868786337 \mathrm{e}+02$ & $1.4123731878 \mathrm{e}+03$ \\
\hline 11.25 & $-1.4035237447 \mathrm{e}-02$ & $3.0135663179 \mathrm{e}-01$ & $6.6855205976 \mathrm{e}+00$ & $2.5402805390 \mathrm{e}+01$ & $1.7805781522 \mathrm{e}+02$ & $1.5207149824 e+03$ \\
\hline 11.5 & $-1.3540370670 \mathrm{e}-02$ & $2.9791032518 \mathrm{e}-01$ & $6.7604264303 e+00$ & $2.6243186856 \mathrm{e}+01$ & $1.8774114745 \mathrm{e}+02$ & $1.6350107444 \mathrm{e}+03$ \\
\hline 11.75 & $-1.3075093467 \mathrm{e}-02$ & $2.9458398150 \mathrm{e}-01$ & $6.83448585844 \mathrm{e}+00$ & $2.7092880634 \mathrm{e}+01$ & $1.9774137139 \mathrm{e}+02$ & $1.7554574360 \mathrm{e}+03$ \\
\hline 12 & $-1.26369084444 \mathrm{e}-02$ & $2.9137052150 \mathrm{e}-01$ & $6.9077279523 \mathrm{e}+00$ & $2.7951777387 \mathrm{e}+01$ & $2.0806195868 \mathrm{e}+02$ & $1.8822542014 \mathrm{e}+03$ \\
\hline 12.25 & $-1.2223585674 \mathrm{e}-02$ & $2.8826345607 \mathrm{e}-01$ & $6.9801800993 e+00$ & $2.8819779746 \mathrm{e}+01$ & $2.1870634264 \mathrm{e}+02$ & $2.0156023426 \mathrm{e}+03$ \\
\hline 12.5 & $-1.1833128745 \mathrm{e}-02$ & $2.8525682375 \mathrm{e}-01$ & $7.0518681393 e+00$ & $2.9696790599 \mathrm{e}+01$ & $2.2967791957 \mathrm{e}+02$ & $2.1557052956 \mathrm{e}+03$ \\
\hline 12.75 & $-1.1463745461 e-02$ & $2.8234513606 \mathrm{e}-01$ & $7.1228164863 e+00$ & $3.0582715975 e+01$ & $2.4098004991 \mathrm{e}+02$ & $2.3027686076 \mathrm{e}+03$ \\
\hline 13 & $-1.1113822964 \mathrm{e}-02$ & $2.7952332972 \mathrm{e}-01$ & $7.1930482379 e+00$ & $3.1477464868 \mathrm{e}+01$ & $2.5261605944 \mathrm{e}+02$ & $2.4569999157 \mathrm{e}+03$ \\
\hline 13.25 & $-1.0781906844 \mathrm{e}-02$ & $2.7678672444 \mathrm{e}-01$ & $7.2625852748 \mathrm{e}+00$ & $3.2380949083 e+01$ & $2.6458924036 e+02$ & $2.6186089248 e+03$ \\
\hline
\end{tabular}




\begin{tabular}{|c|c|c|c|c|c|c|}
\hline$\eta$ & $F^{\prime \prime}{ }_{-1 / 2}$ & $\mathrm{~F}_{-1 / 2}^{\prime}$ & $F_{-1 / 2}$ & $F_{1 / 2}$ & $\mathrm{~F}_{3 / 2}$ & $\mathrm{~F}_{5 / 2}$ \\
\hline 13.5 & $-1.0466683406 \mathrm{e}-02$ & $2.7413098559 \mathrm{e}-01$ & $7.3314483509 e+00$ & $3.3293083094 e+01$ & $2.7690285233 e+02$ & $2.7878073878 \mathrm{e}+03$ \\
\hline 13.75 & $-1.0166964113 \mathrm{e}-02$ & $2.7155209103 \mathrm{e}-01$ & $7.3996571757 \mathrm{e}+00$ & $3.4213783899 \mathrm{e}+01$ & $2.8956012342 \mathrm{e}+02$ & $2.9648090852 \mathrm{e}+03$ \\
\hline 14 & $-9.8816713811 \mathrm{e}-03$ & $2.6904630159 \mathrm{e}-01$ & $7.4672304885 \mathrm{e}+00$ & $3.5142971120 \mathrm{e}+01$ & $3.0256425105 e+02$ & $3.1498298055 e+03$ \\
\hline 14.25 & $-9.6098253758 \mathrm{e}-03$ & $2.6661013502 \mathrm{e}-01$ & $7.5341861261 \mathrm{e}+00$ & $3.6080565984 \mathrm{e}+01$ & $3.1591840283 e+02$ & $3.3430873255 \mathrm{e}+03$ \\
\hline 14.5 & $-9.3505319841 \mathrm{e}-03$ & $2.6424034306 \mathrm{e}-01$ & $7.6005410839 e+00$ & $3.7026480077 e+01$ & $3.2962571735 \mathrm{e}+02$ & $3.5448013921 \mathrm{e}+03$ \\
\hline 14.75 & $-9.1029724808 \mathrm{e}-03$ & $2.6193389139 \mathrm{e}-01$ & $7.6663115723 e+00$ & $3.7980676887 \mathrm{e}+01$ & $3.4368930696 e+02$ & $3.7551937039 e+03$ \\
\hline 15 & $-8.8663951139 \mathrm{e}-03$ & $2.5968794181 \mathrm{e}-01$ & $7.7315130679 e+00$ & $3.8943046768 \mathrm{e}+01$ & $3.5811225020 \mathrm{e}+02$ & $3.9744878942 e+03$ \\
\hline 15.25 & $-8.6401083988 \mathrm{e}-03$ & $2.5749983643 \mathrm{e}-01$ & $7.7961603604 \mathrm{e}+00$ & $3.9913532034 \mathrm{e}+01$ & $3.7289760572 e+02$ & $4.2029095147 \mathrm{e}+03$ \\
\hline 15.5 & $-8.4234762065 \mathrm{e}-03$ & $2.5536708321 \mathrm{e}-01$ & $7.8602675961 e+00$ & $4.0892064292 \mathrm{e}+01$ & $3.8804840379 \mathrm{e}+02$ & $4.4406860348 \mathrm{e}+03$ \\
\hline 15.75 & $-8.2159143691 \mathrm{e}-03$ & $2.5328734260 \mathrm{e}-01$ & $7.9238483174 \mathrm{e}+00$ & $4.1878576884 \mathrm{e}+01$ & $4.0356764948 \mathrm{e}+02$ & $4.6880467644 \mathrm{e}+03$ \\
\hline 16 & $-8.0168868648 \mathrm{e}-03$ & $2.5125841495 \mathrm{e}-01$ & $7.9869154998 \mathrm{e}+00$ & $4.2873004821 \mathrm{e}+01$ & $4.1945832333 e+02$ & $4.9452229375 \mathrm{e}+03$ \\
\hline 16.25 & $-7.8259006576 \mathrm{e}-03$ & $2.4927822916 \mathrm{e}-01$ & $8.0494815850 \mathrm{e}+00$ & $4.3875284707 \mathrm{e}+01$ & $4.3572338193 e+02$ & $5.2124476269 \mathrm{e}+03$ \\
\hline 16.5 & $-7.6425019180 \mathrm{e}-03$ & $2.4734483244 \mathrm{e}-01$ & $8.1115585121 \mathrm{e}+00$ & $4.4885354681 \mathrm{e}+01$ & $4.5236575851 \mathrm{e}+02$ & $5.4899557471 \mathrm{e}+03$ \\
\hline 16.75 & $-7.4662744026 \mathrm{e}-03$ & $2.4545638070 \mathrm{e}-01$ & $8.1731577456 \mathrm{e}+00$ & $4.5903154343 \mathrm{e}+01$ & $4.6938836337 \mathrm{e}+02$ & $5.7779840386 \mathrm{e}+03$ \\
\hline 17 & $-7.2968340617 \mathrm{e}-03$ & $2.4361112970 \mathrm{e}-01$ & $8.2342903016 \mathrm{e}+00$ & $4.6928624695 \mathrm{e}+01$ & $4.8679408431 \mathrm{e}+02$ & $6.0767710514 \mathrm{e}+03$ \\
\hline 17.25 & $-7.1338177286 \mathrm{e}-03$ & $2.4180742837 \mathrm{e}-01$ & $8.2949667721 e+00$ & $4.7961708073 \mathrm{e}+01$ & $5.0458578700 \mathrm{e}+02$ & $6.3865571285 e+03$ \\
\hline 17.5 & $-6.9768777049 \mathrm{e}-03$ & $2.4004371454 \mathrm{e}-01$ & $8.3551973474 \mathrm{e}+00$ & $4.9002348095 \mathrm{e}+01$ & $5.2276631545 \mathrm{e}+02$ & $6.7075843930 \mathrm{e}+03$ \\
\hline 17.75 & $-6.8256920250 \mathrm{e}-03$ & $2: 3831851010 \mathrm{e}-01$ & $8.4149918379 e+00$ & $5.0050489623 \mathrm{e}+01$ & $5.4133849265 \mathrm{e}+02$ & $7.0400967368 \mathrm{e}+03$ \\
\hline 18 & $-6.6799749708 \mathrm{e}-03$ & $2.3663041288 \mathrm{e}-01$ & $8.4743596943 e+00$ & $5.1106078715 e+01$ & $5.6030512102 e+02$ & $7.3843398076 \mathrm{e}+03$ \\
\hline
\end{tabular}




\begin{tabular}{||r|c|c|c|c|c|c|}
\hline \multicolumn{1}{|c|}{$\eta$} & $F_{-1 / 2}^{\prime \prime}$ & $F_{-1 / 2}^{\prime}$ & $F_{-1 / 2}$ & $F_{1 / 2}$ & $F_{3 / 2}$ & $F_{5 / 2}$ \\
\hline 18.25 & $-6.5394624048 \mathrm{e}-03$ & $2.3497808887 \mathrm{e}-01$ & $8.5333100251 \mathrm{e}+00$ & $5.2169062574 \mathrm{e}+01$ & $5.7966898268 \mathrm{e}+02$ & $7.7405609927 \mathrm{e}+03$ \\
\hline 18.5 & $-6.4038800873 \mathrm{e}-03$ & $2.3336027076 \mathrm{e}-01$ & $8.5918516138 \mathrm{e}+00$ & $5.3239389498 \mathrm{e}+01$ & $5.9943283974 \mathrm{e}+02$ & $8.1090094054 \mathrm{e}+03$ \\
\hline 18.75 & $-6.2729400631 \mathrm{e}-03$ & $2.3177576205 \mathrm{e}-01$ & $8.6499929359 \mathrm{e}+00$ & $5.4317008861 \mathrm{e}+01$ & $6.1959943523 \mathrm{e}+02$ & $8.4899358833 \mathrm{e}+03$ \\
\hline 19 & $-6.1463871634 \mathrm{e}-03$ & $2.3022343539 \mathrm{e}-01$ & $8.7077421765 \mathrm{e}+00$ & $5.5401871129 \mathrm{e}+01$ & $6.4017149441 \mathrm{e}+02$ & $8.8835929927 \mathrm{e}+03$ \\
\hline 19.25 & $-6.0240476450 \mathrm{e}-03$ & $2.2870221755 \mathrm{e}-01$ & $8.7651072459 \mathrm{e}+00$ & $5.6493927843 \mathrm{e}+01$ & $6.6115172545 \mathrm{e}+02$ & $9.2902350211 \mathrm{e}+03$ \\
\hline 19.5 & $-5.9058064296 \mathrm{e}-03$ & $2.2721106994 \mathrm{e}-01$ & $8.8220957910 \mathrm{e}+00$ & $5.7593131537 \mathrm{e}+01$ & $6.8254281873 \mathrm{e}+02$ & $9.7101179432 \mathrm{e}+03$ \\
\hline 19.75 & $-5.7915111364 \mathrm{e}-03$ & $2.2574898516 \mathrm{e}-01$ & $8.8787152026 \mathrm{e}+00$ & $5.8699435620 \mathrm{e}+01$ & $7.0434744558 \mathrm{e}+02$ & $1.0143499379 \mathrm{e}+04$ \\
\hline 20 & $-5.6809001639 \mathrm{e}-03$ & $2.2431500714 \mathrm{e}-01$ & $8.9349726255 \mathrm{e}+00$ & $5.9812794314 \mathrm{e}+01$ & $7.2656825838 \mathrm{e}+02$ & $1.0590638586 \mathrm{e}+04$ \\
\hline
\end{tabular}

点 
Table 4-3. Comparison between $F_{-3 / 2}, F_{-1 / 2}^{\prime}$ and $\mathscr{F}_{-3 / 2}$ [7]. The last three columns contain the ratios of the numbers, note that for $\mathrm{F}_{-3 / 2} / \mathrm{F}_{-1 / 2}$ and $\mathrm{F}_{-3 / 2} / \mathscr{I}_{-3 / 2}$ are not constant leading to the conclusion that these functions are not related. On the other hand $F_{-1 / 2}^{\prime} / \mathscr{F}_{-3 / 2}$ is a constant and equal to $(\pi)^{1 / 2}$.

\begin{tabular}{|r|r|r|r|r|r|r||}
\hline$\eta$ & \multicolumn{1}{|c|}{$\mathrm{F}_{-3 / 2}$} & \multicolumn{1}{|c|}{$\mathrm{F}_{-1 / 2}^{\prime}$} & \multicolumn{1}{|c|}{$\mathcal{F}_{-3 / 2}[7]$} & $\mathrm{F}_{-3 / 2} / \mathrm{F}_{-1 / 2}^{\prime}$ & \multicolumn{1}{|c|}{$\mathrm{F}_{-3 / 2} / \mathscr{F}_{-3 / 2}$} & \multicolumn{1}{|c|}{$\mathrm{F}_{-1 / 2} / \mathscr{I}_{-3 / 2}$} \\
\hline-2.5 & 62.649017136 & 0.13015291246 & 0.0735 & 481.3493 & 852.3676 & 1.770788 \\
\hline 0 & 413.30344852 & 0.67371823886 & 0.38 & 613.4663 & 1087.641 & 1.772943 \\
\hline 2.5 & 765.01225594 & 0.6900955385 & 0.3893 & 1108.56 & 1965.097 & 1.772657 \\
\hline 5 & 822.79829306 & 0.47654190047 & 0.269 & 1726.602 & 3058.73 & 1.771531 \\
\hline 10 & 828.62274814 & 0.32068665358 & 0.181 & 2583.902 & 4578.026 & 1.771749 \\
\hline
\end{tabular}




\section{REFERENCES}

1. R. Kishore, 'Generalized Equations for the Steady-State Analysis of Inhomogeneous Semiconductor Devices', Solid-State Elec., Vol. 33, \#8, pp. 1049-54, (1990).

2. E.M. Azoff, 'Generalized Energy-Momenutm Conservation Equations in the Relaxation Time Approximation', Solid-State Elec., Vol. 33, \#8, pp. 1049-54, (1990).

3. J. McDougall and E.C. Stoner, 'The Computation of Fermi-Dirac Functions', Phil. Trans. Roy. Soc. A, Vol. 237, pp. 67-104, (1938).

4. A.C. Beer, M.N. Chase, and P.F. Choquard, 'Extension of McDougall-Stoner Tables of the FermiDirac Functions', Helv. Phys. Acta, Vol. 28, pp. 529-42, (1955).

5. R.B. Dingle, 'The Fermi-Dirac Integrals', Appl. Sci. Res. B, Vol 6, pp. 225-39, (1957).

6. W.H. Beyer, Standard Mathematical Tables $28^{\text {th }}$ Ed., CRC Press, Boca Raton, FL., (1987).

7. J.S. Blakemore, Semiconductor Statistics, Dover Publications Inc., New York, (1987).

8. L.D. Cloutman, 'Numerical Evaluation of the Fermi-Dirac Integrals', Astrophysical Journal Supplement Series, Vol. 71, pp. 677-99, (1989).

9. W.H. Press, B.P. Flannery, S.A. Teukolsky and W.T. Vetterling, Numerical Recipes The Art of Scientific Computing, Cambridge University Press, Cambridge, (1986). 


\section{SILICON SOLAR CELL FABRICATION}

\subsection{PROCESS DEVELOPMENT}

High carrier lifetime is the key to efficient solar cells. Silicon growth technology has matured enough to give lifetime in the ms range. However, maintaining high lifetime through solar cell fabrication is a challenge and requires contaminant free diffusion and oxidation furnace tubes, clean processing, and careful handling procedures. TCA gettering is the best way to keep the furnace tubes clean. First, TCA gettering, oxidation, diffusions, and Al heat treatment steps were developed to preserve high lifetime. Then, theoretical and experimental development of two-layer AR coating was carried out. Finally, solar cells were fabricated on $F Z, M C Z$, and $C Z$ silicon with and without $A R$ coating.

\section{TCA GETTERING OF THE FURNACE TUBES}

Trichloroethane (TCA) is effective in furnace tube cleaning because at a temperature greater than $400^{\circ} \mathrm{C}, T C A$ oxidizes to produce high purity hydrogen chloride $(\mathrm{HCl})$, according to the following reaction

$$
\mathrm{CH}_{3} \mathrm{CCL}_{3}+2 \mathrm{O}_{2}-3 \mathrm{HCL}+2 \mathrm{CO}_{2}
$$

The stoichiometric amount of $\mathrm{O}_{2}$ is used to prevent the formation of carbon by interlocking the carrier $\mathrm{N}_{2}$ (through the TCA Bubbler)to the $\mathrm{O}_{2}$ flow. Gettering of the lifetime-limiting metallic contaminants in the furnace tube takes place because 
contaminants at the surface of the tube react with the chlorine to form volatile metal chlorides, which are transported out of the furnace tube. In addition sodium contamination from the furnace tube, boats and pull rod is removed by TCA gettering.

Before placing a new tube in the furnace, the tube is cleaned in aqua regia (3:1 $\mathrm{HCL}: \mathrm{HNO}_{3}$ ), followed by $\mathrm{DI}$ rinse, 20:1 Dl:HF rinse and another $\mathrm{DI}$ rinse. Then the tube is TCA gettered for six hours at $1050^{\circ} \mathrm{C}$.

\section{OXIDATION OF SILICON}

We use dry oxidation for AR coating $\left(1100 \mathrm{~A}^{\circ}\right)$, passivation $\left(100 \mathrm{~A}^{\circ}\right)$, and diffusion barriers $\left(2000 \mathrm{~A}^{\circ}\right)$. Prior to any oxidation step, the furnace is TCA gettered for about an hour at $1050^{\circ} \mathrm{C}$. We are able to maintain wafer lifetime in excess of $2 \mathrm{~ms}$ during the dry oxidation steps. We have been able to achieve as high as $5 \mathrm{~ms}$ lifetime after one hour of TCA oxidation at $1000^{\circ} \mathrm{C}$. Proper wafer cleaning was critical in achieving high lifetime. The following wafer cleaning steps were used

* Dl rinse $3 \mathrm{~min}$

* $\mathrm{H}_{2} \mathrm{SO}_{4}: \mathrm{H}_{2} \mathrm{O}_{2} 4: 15 \mathrm{~min}$

* Dl rinse $3 \mathrm{~min}$

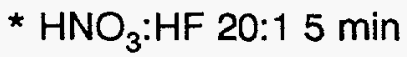

* DI rinse $3 \mathrm{~min}$

* Dl: $\mathrm{H}_{2} \mathrm{O}_{2}: \mathrm{HCL} \quad 5: 2: 210 \mathrm{~min}$

* Dl rinse $3 \mathrm{~min}$

* $\mathrm{H}_{2} \mathrm{O}: \mathrm{HF} 10: 13 \mathrm{~min}$

* DI rinse $3 \mathrm{~min}$ 


\section{DIFFUSIONS}

Diffusion furnaces prior to introducing the solid sources were TCA gettered according to the same process described above. The oxidation in our diffusion furnaces, without any dopant source, can maintain high lifetime of the order of $2 \mathrm{~ms}$. However, as discussed below, presence of solid dopant sources in the diffusion furnace degrades the lifetime in the case of boron diffusion.

\section{Phosphorous Diffusion}

We decided to use solid sources because they are safe, require minimum care and handling, and are very convenient to use in a university research lab. Phosphorous solid sources were bought from Owens-Illinois and Carborundum Company. Phosphorous Plus sources from Owens-llinois were not able to maintain high lifetime. On the other hand, PH-950 planar diffusion source from Carborundum was able to give $2 \mathrm{~ms}$ lifetime after 30 minutes phosphorous diffusion at $900^{\circ} \mathrm{C}$. Therefore, $\mathrm{PH}-950$ planar source is being used in our solar cell fabrication.

\section{Boron Diffusion}

Boron sources were obtained from Owens-lllinois and Carborundum (BN-975) Company. Both of these sources could not maintain the high lifetime in the wafer after boron diffusion. We are now investigating ultra-high-purity Boron diffusion sources from Owens-Illinois. If high lifetime cannot be maintained by boron solid source diffusion, we will consider using liquid source or $\mathrm{Al}$ heat treatment for $\mathrm{p}^{+}$region. 


\section{Aluminum Heat Treatment}

Al treatment is being investigated to replace boron diffusion. About 1-um thick aluminum is evaporated on the back of the silicon solar cells, followed by a $600^{\circ} \mathrm{C}$ forming gas anneal for 30 minutes. Preliminary cell data shows that rear aluminum contact gives better cell performance than boron back surface field cells fabricated on .2 ohm-cm p-type wafers. It appears that aluminum treatment getters some of the impurities and gives higher bulk lifetime. More research is underway to understand the aluminum treatment and the gettering processes associated with it.

\section{TEXTURING AND THINNING PROCESSES}

Wafer thinning and texturing are two important processes to improve the performance of solar cells.

\section{Wafer Thinning Procedure}

1. Heat the $\mathrm{KOH} 45 \%$ solution to $110^{\circ} \mathrm{C}$.

2. Place wafers in a teflon boat and immerse in the $\mathrm{KOH}$ solution.

3. The etch rate was found to be $3 u \mathrm{~m} / \mathrm{min}$ per side.

\section{Surface Texturing Process}

Surface texturing reduces reflectance, increases the absorption of long wavelength photons, and traps light to give high current. Careful processing is required to achieve uniform textured surfaces with high lifetime in the wafer. The following procedure was developed for pyramid-like texturing of the surface. 
* Place $500 \mathrm{ml} \mathrm{Dl}$ water in a quartz beaker

* Add $8 \mathrm{ml} \mathrm{KOH}$

* Stir and cover the beaker with a watch glass

* Heat the solution to $65^{\circ} \mathrm{C}$

* Remove the watch glass and add $40 \mathrm{ml}$ Isopropyl Alcohol (IPA) and use a boat with a dummy wafer to stir the IPA

* Leave the boat in the texturing solution and cover the beaker

* Raise the temperature to $85^{\circ} \mathrm{C}$

* Remove the dummy wafer

* Immerse the wafers to be textured in the solution

* Cover the beaker

* Leave wafers for about 50-60 minutes to get random pyramid texture over the entire wafer

\section{Post-Texture Clean}

It is very important to remove the residual potassium from the surface after the texturing. The following procedure was developed to chemically oxidize the surface and then etch the oxide layer to remove the residual potassium.

* DI Rinse for 5 minutes

* Immerse the textured wafers in $\mathrm{H}_{2} \mathrm{SO}_{4}: \mathrm{H}_{2} \mathrm{O}_{2} 4: 1$ for about 10 minutes

* DI Rinse for 5 minutes

* 100:1 $\mathrm{HNO}_{3}: \mathrm{HF} \mathrm{5-10} \mathrm{sec} \mathrm{dip}$

* DI Rinse for 5 minutes

* Dl: $\mathrm{H}_{2} \mathrm{O}_{2}: \mathrm{HCl}$ 5:2:2 for 10 minutes 
* DI Rinse for 5 minutes

* DI:HF 10:1 for 4 minutes

* DI Rinse for 5 minutes

Above procedure was found to maintain the initial carrier lifetime in the wafers.

\subsection{DEVELOPMENT OF ANTIREFLECTION COATINGS}

Good AR coating increases the short-circuit current of a solar cell by reducing the surface reflectance. In this report, we used thin film design software and the AM1.5 spectrum to optimize the AR coating design for a wavelength range of 0.3 to $1.1 \mathrm{um}$. The AR coating model (CAMS) used in this study is briefly described in appendix C. Calculations were performed using the model to design and optimize a two-layer AR coating with $\mathrm{ZnS} / \mathrm{MgF}_{2}$ and $\mathrm{TiO}_{2} / \mathrm{SiO}_{2}$. The theoretical calculations (Figures 5-2 to 5-7) indicate that minimum reflectance from $\mathrm{MgF}_{2}$ and $\mathrm{ZnS}$ layers on top of a $100 \mathrm{~A}^{\circ} \mathrm{SiO}_{2}$ is attained with $550 \mathrm{~A}^{\circ} \mathrm{ZnS}$ and $1100 \mathrm{~A}^{\circ} \mathrm{MgF}_{2}$. The experimental data for three different double layer AR coatings, deposited by thermal evaporation of $550 \mathrm{~A}^{\circ}$ of $\mathrm{ZnS}$ and 1100 $\mathrm{A}^{\circ}$ of $\mathrm{MgF}_{2}$ at Georgia Tech, are shown in Figure 5-1. Fairly good agreement was obtained between the calculated and measured values.

$\mathrm{TiO}_{2} / \mathrm{SiO}_{2}$ is a more practical AR coating. Figures 5-8 to 5-11 show the theoretical calculations to achieve minimum reflectance from the two layer $\mathrm{TiO}_{2} / \mathrm{SiO}_{2}$ coating. Model calculations show that minimum reflectance is obtained with $500 \mathrm{~A}^{\circ} \mathrm{TiO}_{2}$ and 1000 $\mathrm{A}^{\circ} \mathrm{SiO}_{2}$. Attempts are being made to deposit this double layer AR coating by thermal evaporation of $\mathrm{TiO}_{2}$ and $\mathrm{SiO}_{2}$.

Finally, theoretical calculations were performed for single layer AR coatings using $\mathrm{SiO}_{2}$ and $\mathrm{Si}_{3} \mathrm{~N}_{4}$ films on silicon. Figures 5-12 and 5-13 show the effects of varying $\mathrm{Si}_{3} \mathrm{~N}_{4}$ and 
si/zns/mgf2

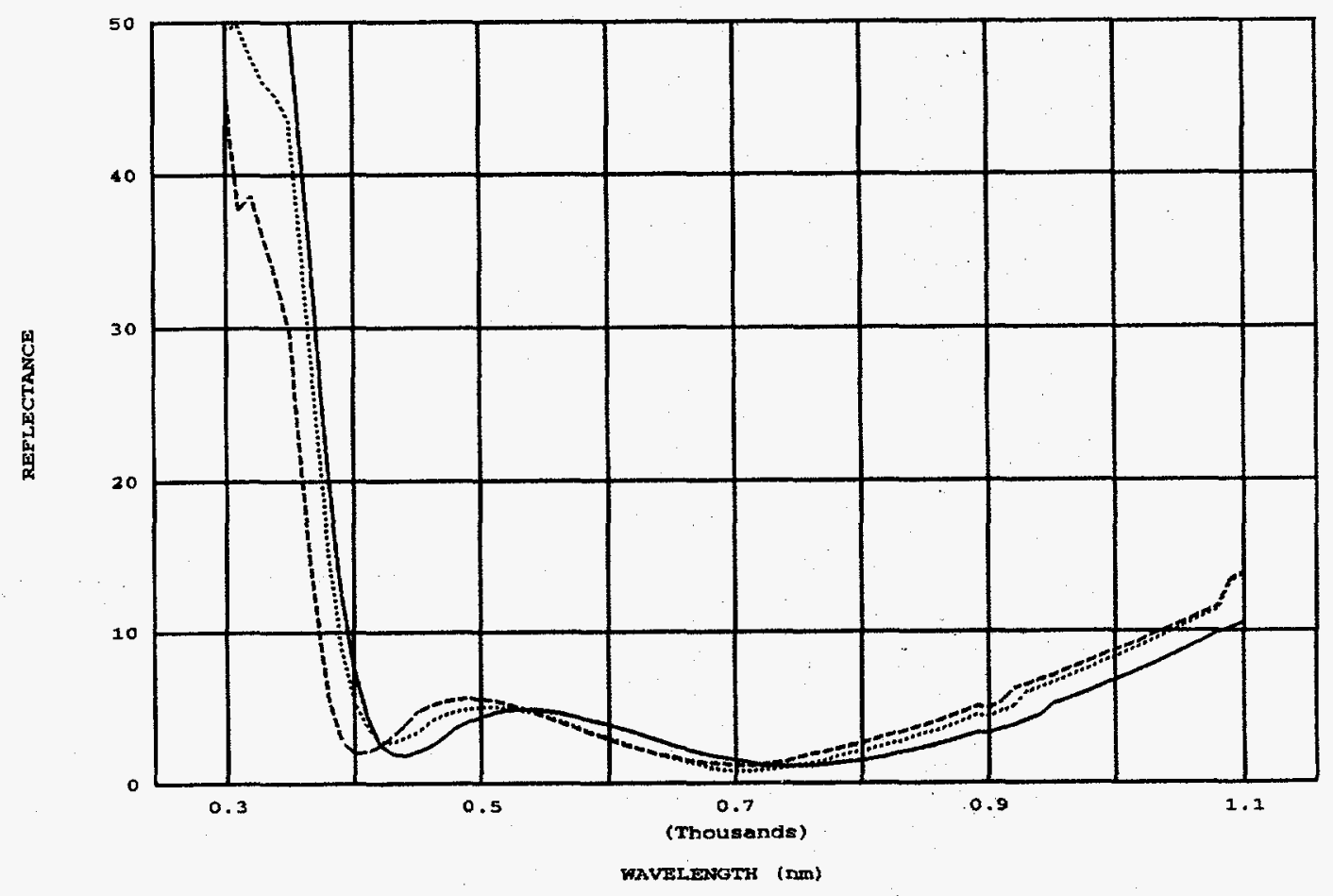

Figure 5-1. Experimental results for deposition of $550 \mathrm{~A} \mathrm{ZnS}$ and $1100 \mathrm{MgF}_{2}$ 
passivating oxide thickness. Best results are obtained with $750 \mathrm{~A}^{\circ} \mathrm{Si}_{3} \mathrm{~N}_{4}$ on top of 100 $A^{\circ}$ passivating oxide. Figure 5-14 shows that $1100 \mathrm{~A}^{\circ} \mathrm{SiO}_{2}$ gives the best single layer AR coating. Figures 5-16 and 5-17 show the effect of angle of incidence on the reflectance of a single layer $\mathrm{Si}_{3} \mathrm{~N}_{4}$ coating. It appears that surface texturing does not warrant a change in the AR coating thickness. 


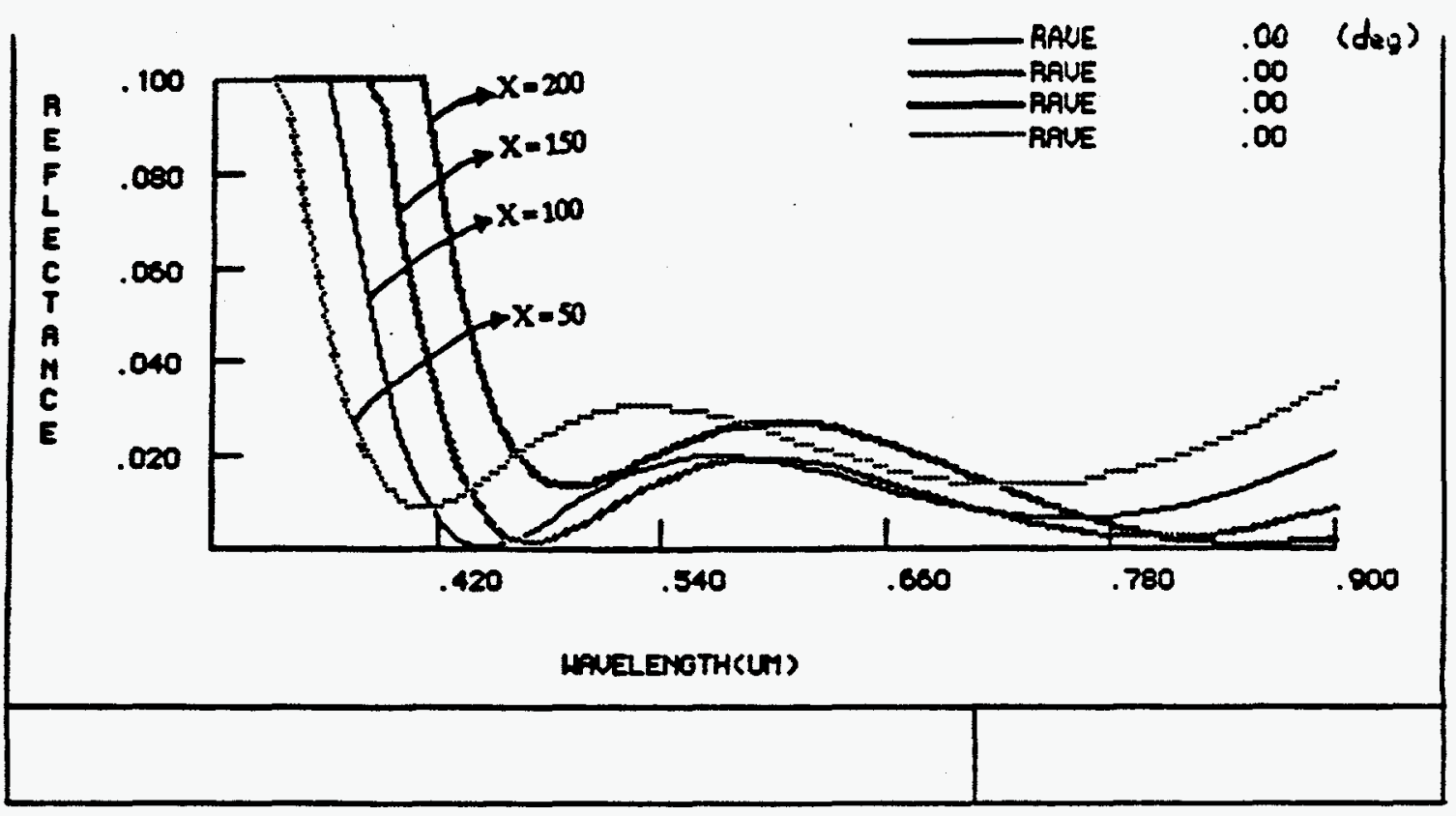

Figure 5-2. Keeping MgF2 and ZoS thickness constant and Changing the SiO2 thickness to check the effect on the reflectance.

Mgrz thickness: 1100 A

ZnS thictoness: 500 A

SiO2 thickeses: $X$ A

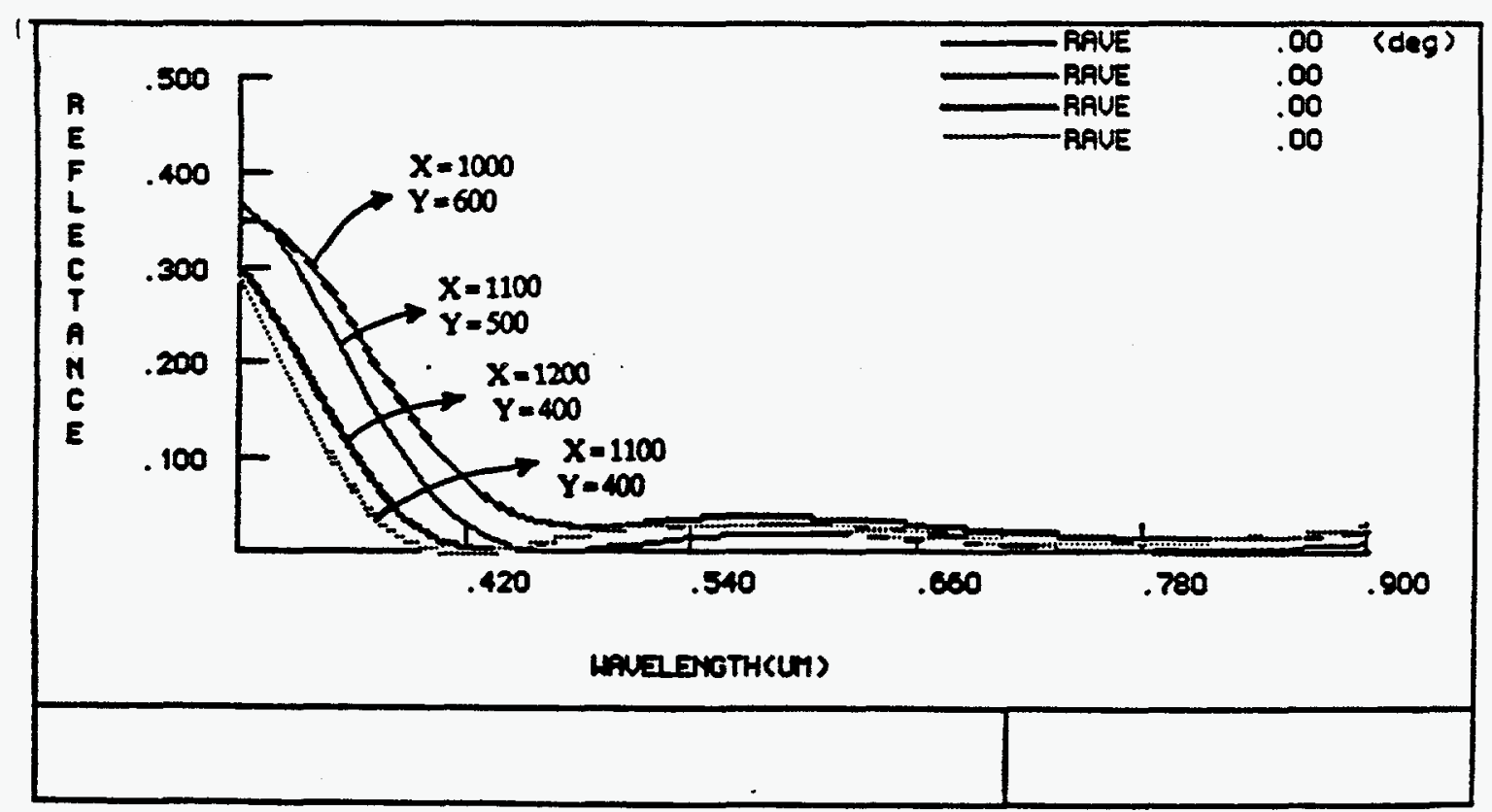

Figure 5-3. Keeping SiO2 thickness constant and Changing the MgF2 and $\mathrm{ZnS}$ thicknesses to see the effect on refleatance.

SiO2 thickoes: $100 \mathrm{~A}$

MgF 2 thicknese: $X$ A

ZoS thickoess: Y A 


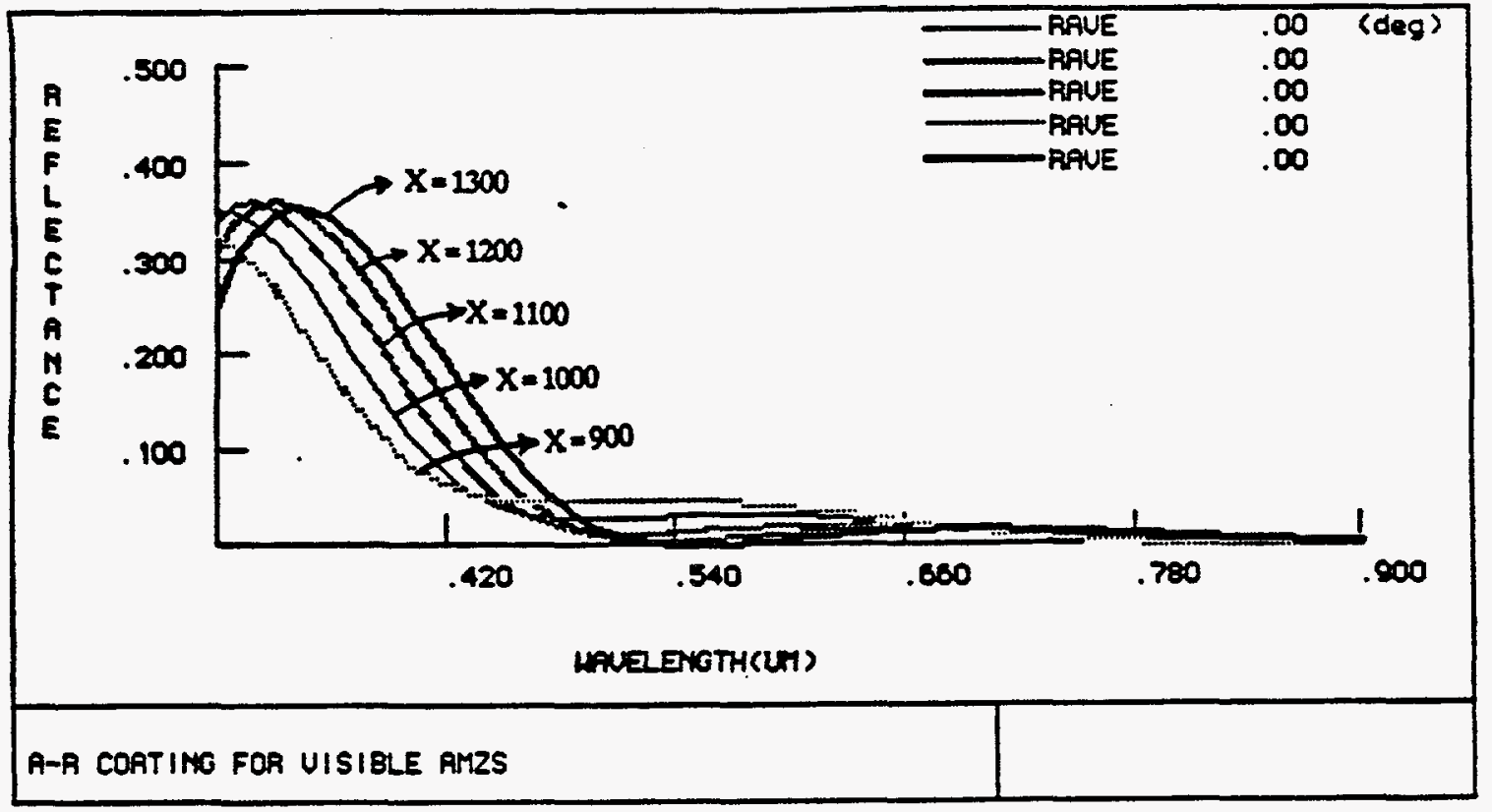

Figure 5-4. Keeping ZnS and SiO2 thicknesses constant and changing the $M g F 2$ thicknesses to see the effed on reflectance.

SiO2 thickness: $100 \mathrm{~A}$

ZnS thickness: $600 \mathrm{~A}$

MgF2 thickness: X A

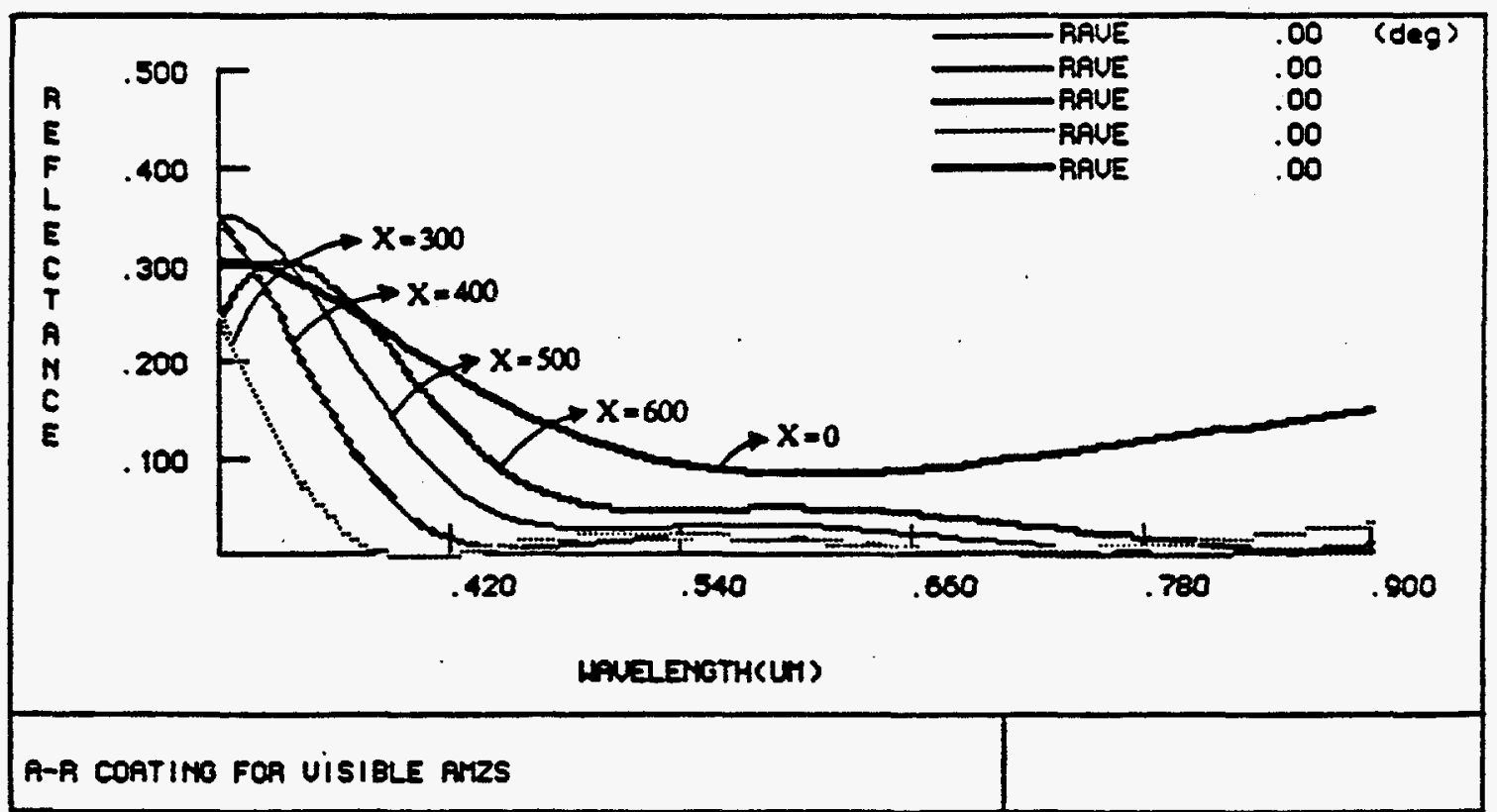

Figure 5-5. Keeping $\mathrm{MgF}_{2}$ and $\mathrm{SiO}_{2}$ thickness constant and Changing the $\mathrm{ZnS}$ thictoness to check the effect on the reflectance.

$\mathrm{MgF}$, thicknese: $1000 \mathrm{~A}$

ZnS thickoess : $X \quad A$

$\mathrm{SiO}_{2}$ thickness: 100 A 


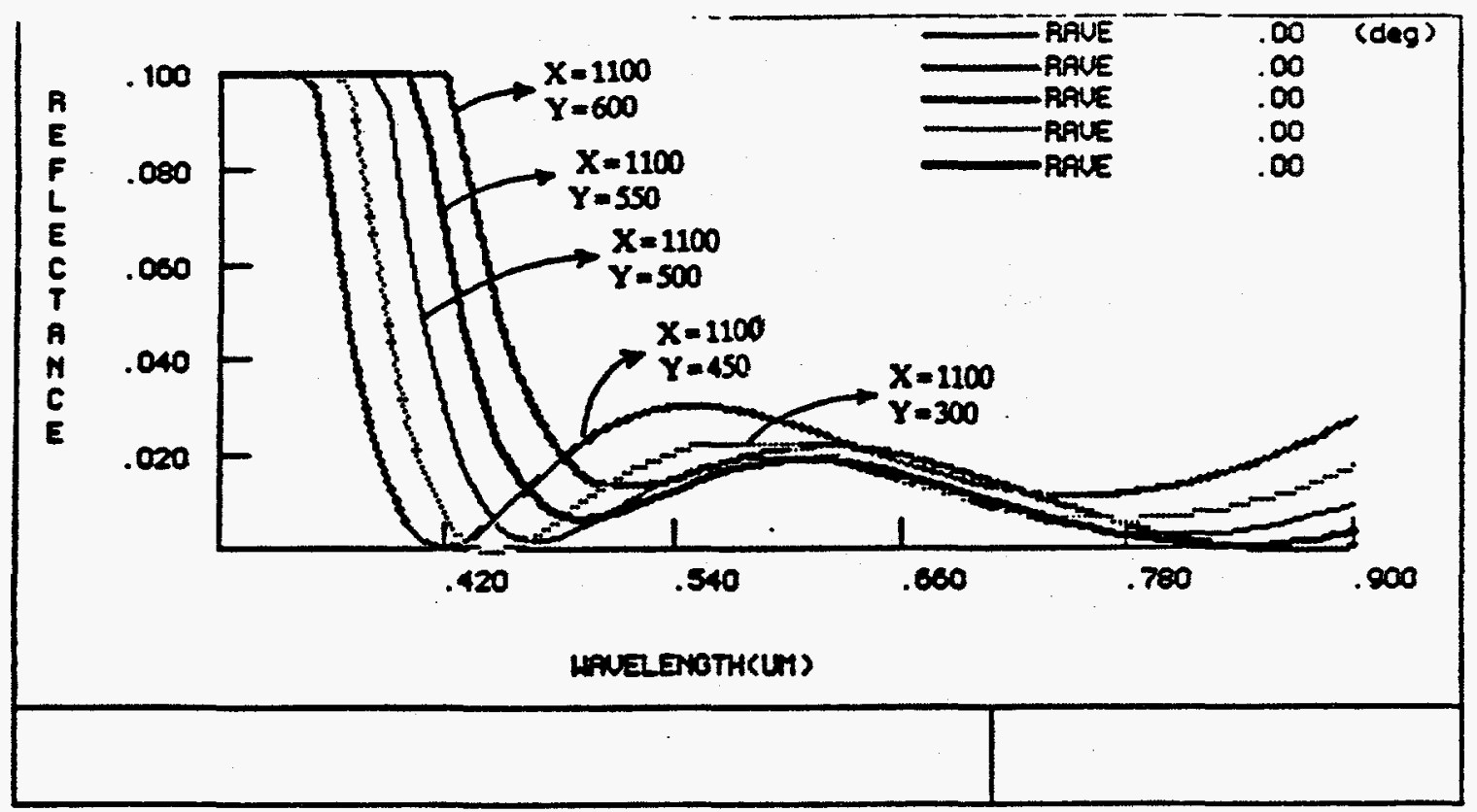

Flgure 5-6. Keeping $\mathrm{SiO}_{2}$ thickness coastant and Changing the $\mathrm{MgF}_{2}$ and Zns thicknesces to see the effect on reflectance.

$\mathrm{SiO}_{2}$ thickness: $100 \mathrm{~A}$

$\mathrm{MgF}_{2}$ thickness X A

ZnS thicknese $Y$ A

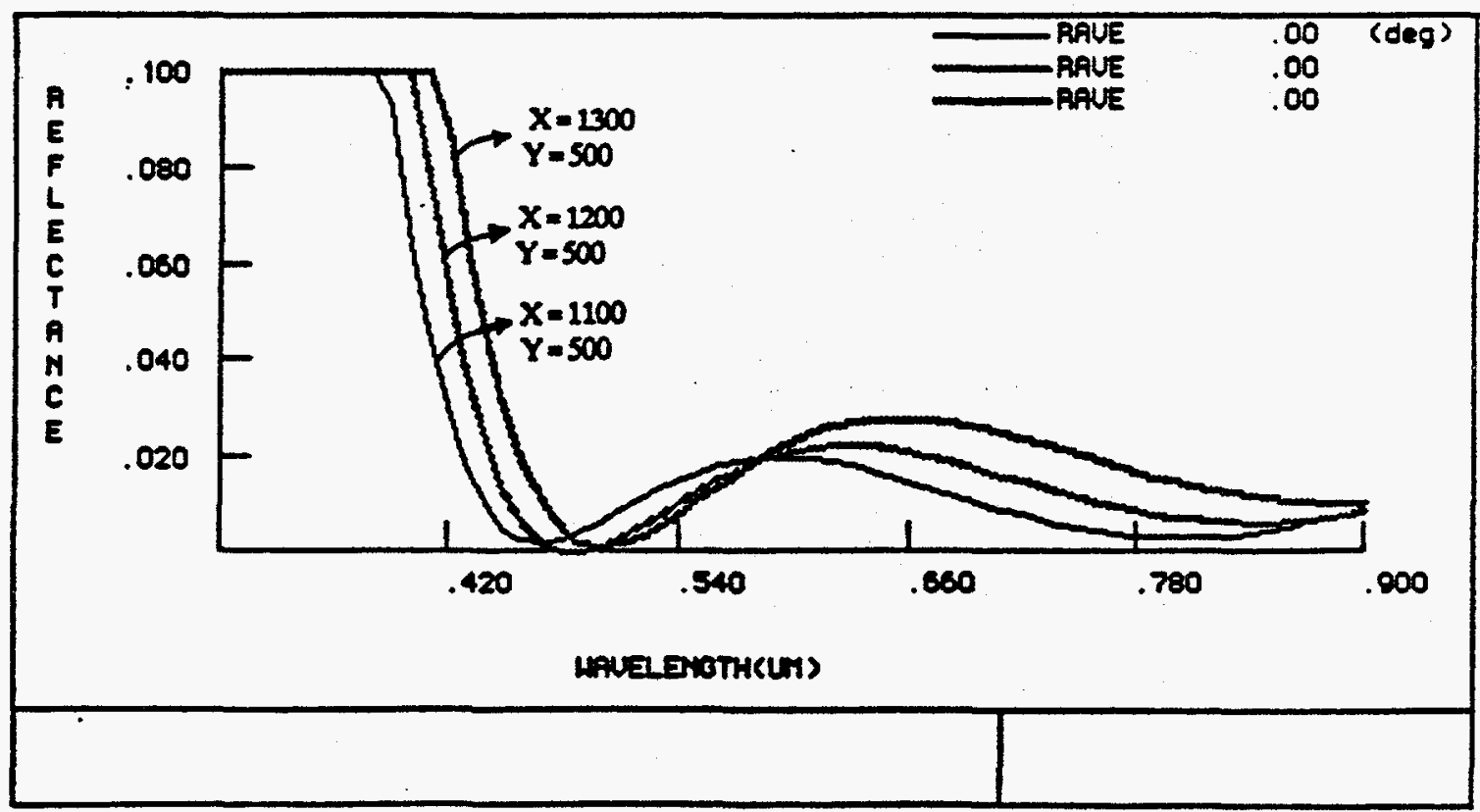

Figure 5-7. Keepiag SiO $\mathrm{O}_{2}$ thickness constant and Changing the $\mathrm{MpF}_{3}$ and Zns thichnesses to see the effect on reflectance.

SiO, thicknese: $100 \mathrm{~A}$

MgF, thicknese: X A

ZnS thickneses: $Y$ A 


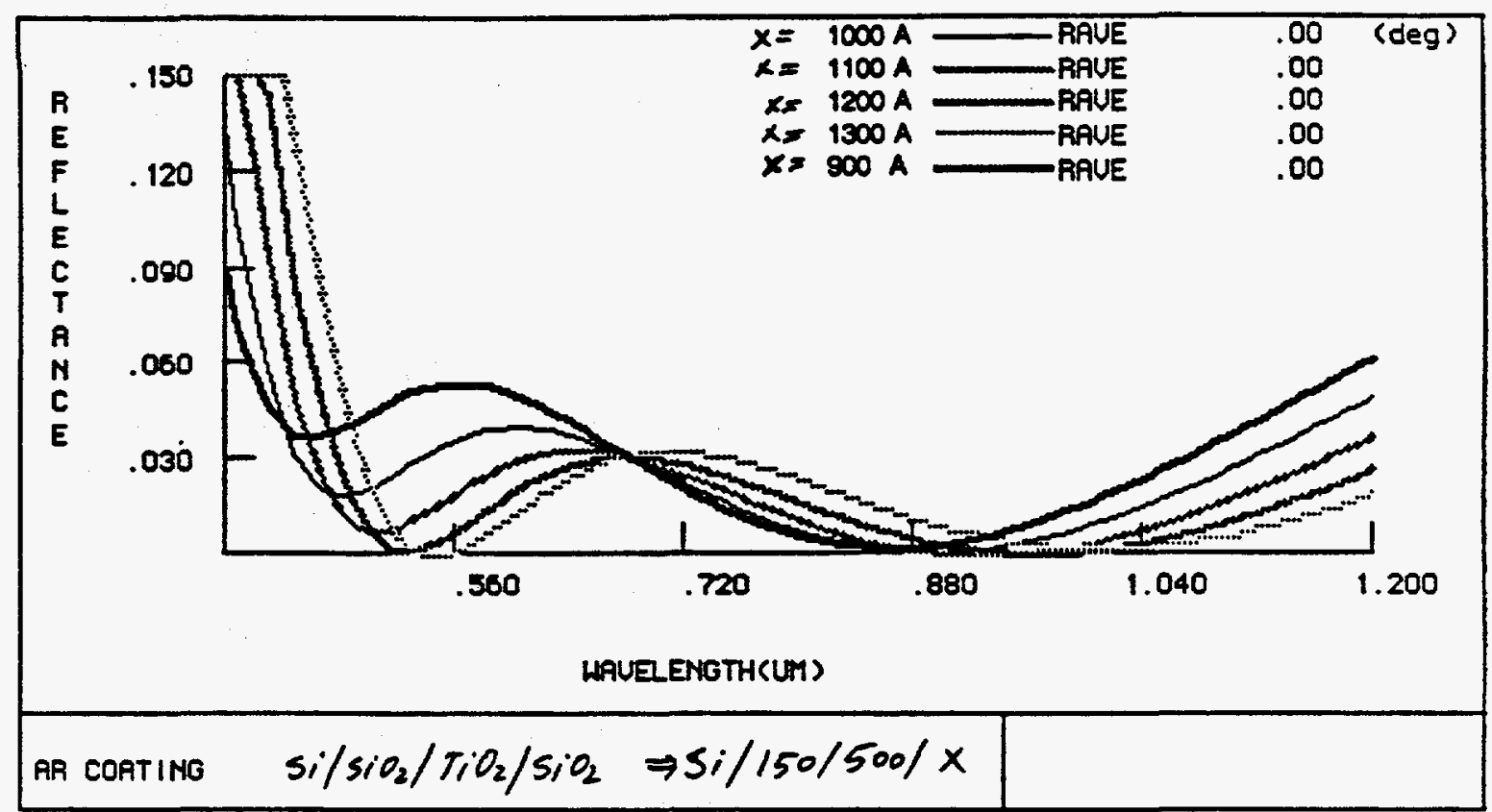

Figure 5-8. Keeping $\mathrm{TIO}_{2}$ and passivating SiO2 thickness constant and changing the SiO2 thickness to check the effect on the reflectance.

TIO2 thickness: $500 \mathrm{~A}$

Passivating SiO2 thickness: $150 \mathrm{~A}$

SiO2 thickness: $\quad \times A$

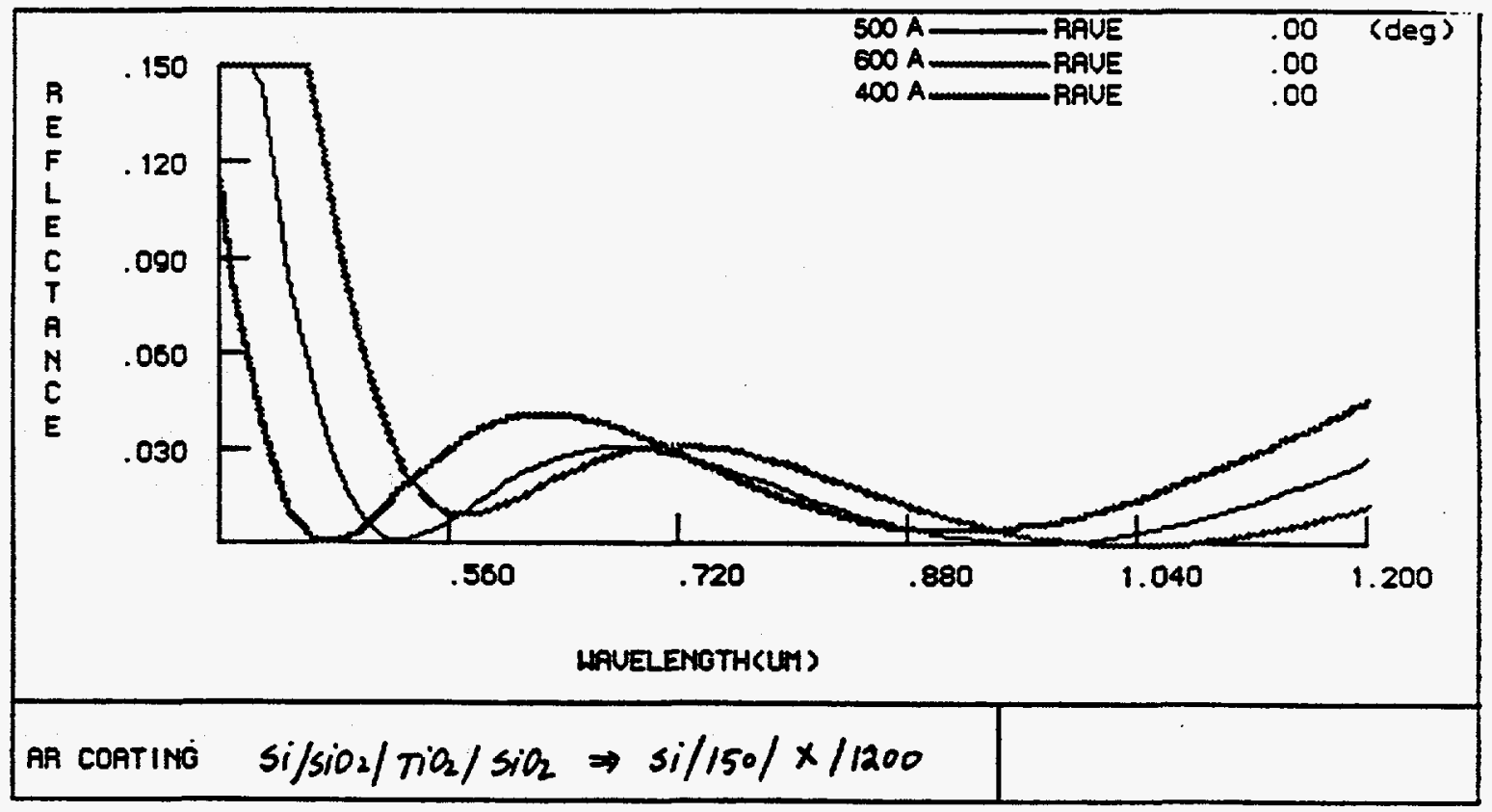

Figure 5-9. Keeping $\mathrm{SiO} 2$ and passivating $\mathrm{SiO} 2$ thickness constant and changing the TIO2 thickness to check the effect on the reflectance.

SiO2 thickness: $1200 \mathrm{~A}$

Passivating SiO2 thickness: $150 \mathrm{~A}$

TIO2 thickness: $X \mathrm{~A}$ 


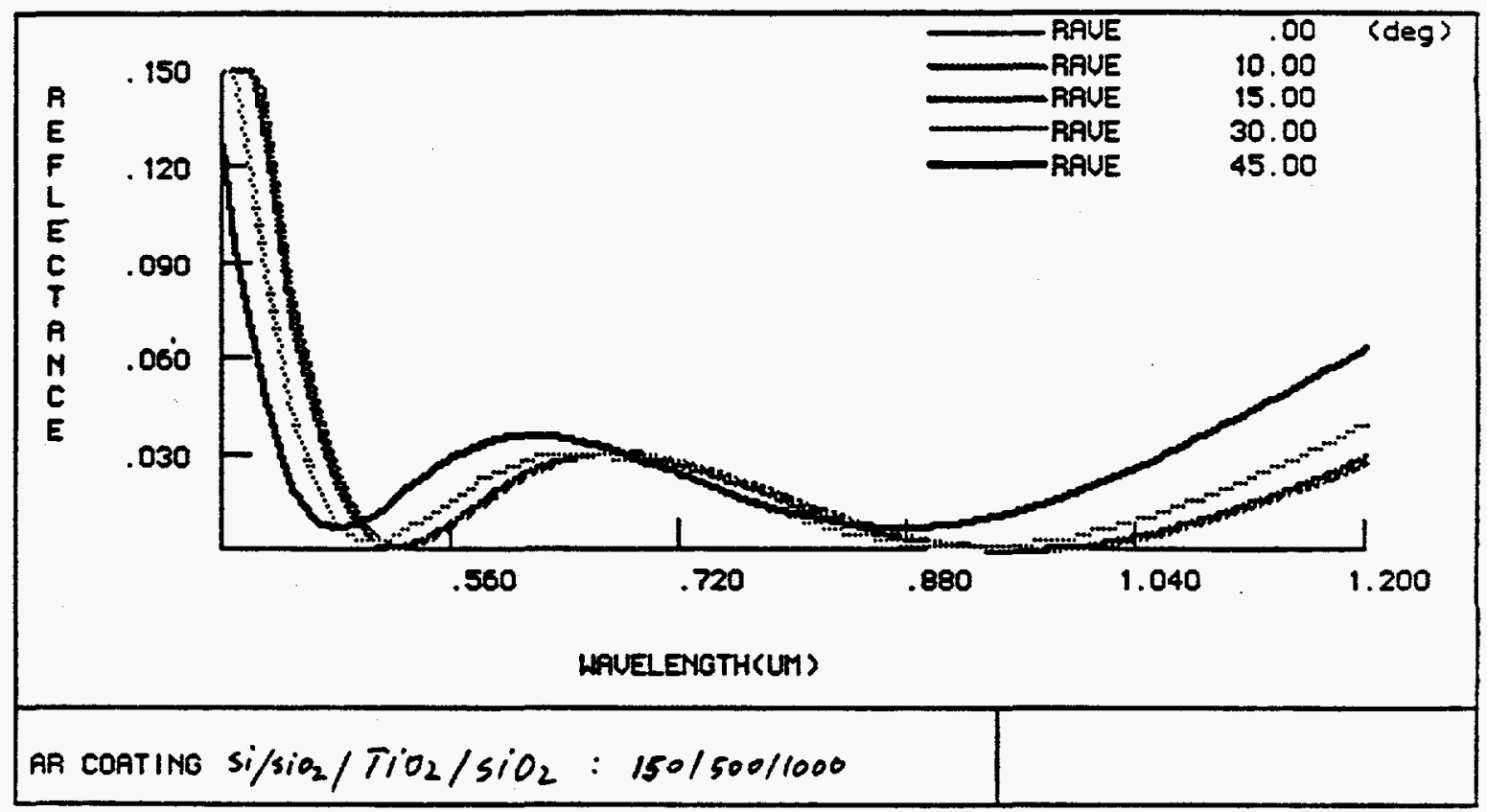

Figure 5-10. Changing the incident angle to check the effect on the refiectance.

SiO2 thickness: $1000 \mathrm{~A}$

TIO2 thickness: 500 A

Passivating SiO2 thickness: $150 \mathrm{~A}$

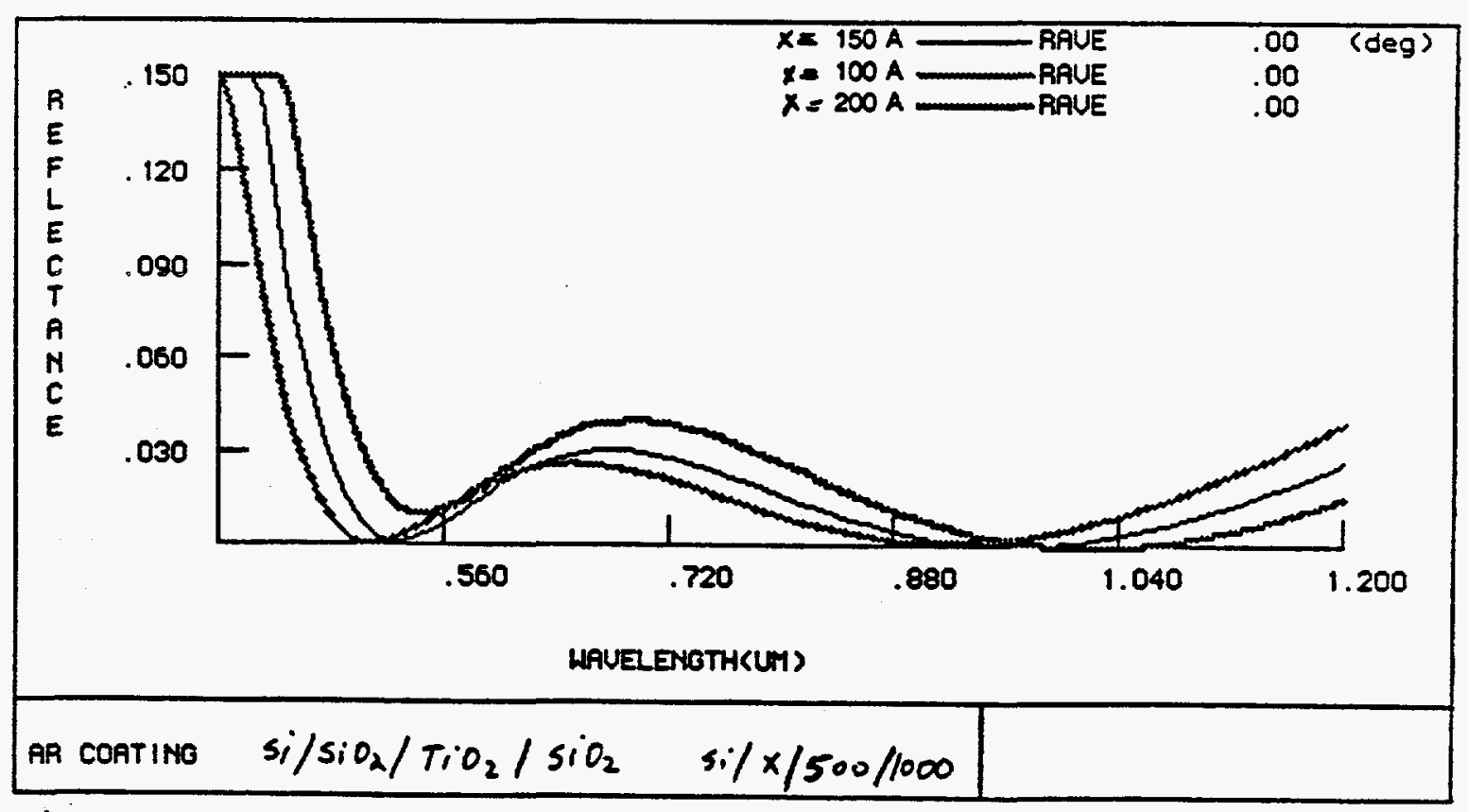

Figure 5-11. Keeping SiO2 and TIO2 thickness constant and changing the passivating $\mathrm{SiO} 2$ thickness to check the effect on the reflectance.

SiO2 thickness: $1000 \mathrm{~A}$

TIO2 thickness: $500 \mathrm{~A}$

Passivating SiO2 thickness: $\times$ A 


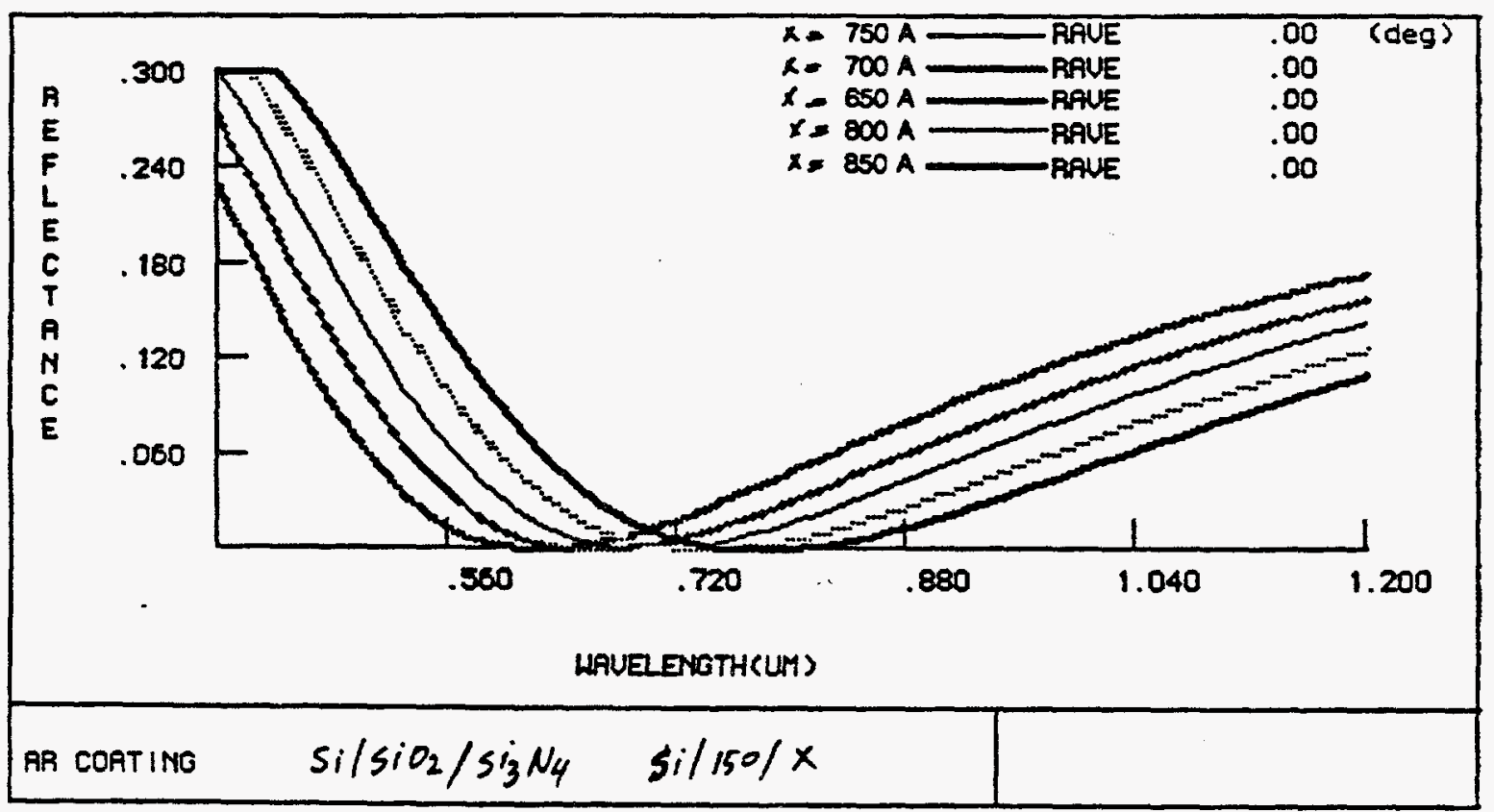

Figure 5-12. Keeping SiO2 thickness constant and changing the Si3N4 thickness to check the effect on

the reflectance.

SiO2 thickness: $150 \mathrm{~A}$

Si3N4 thickness: $X A$

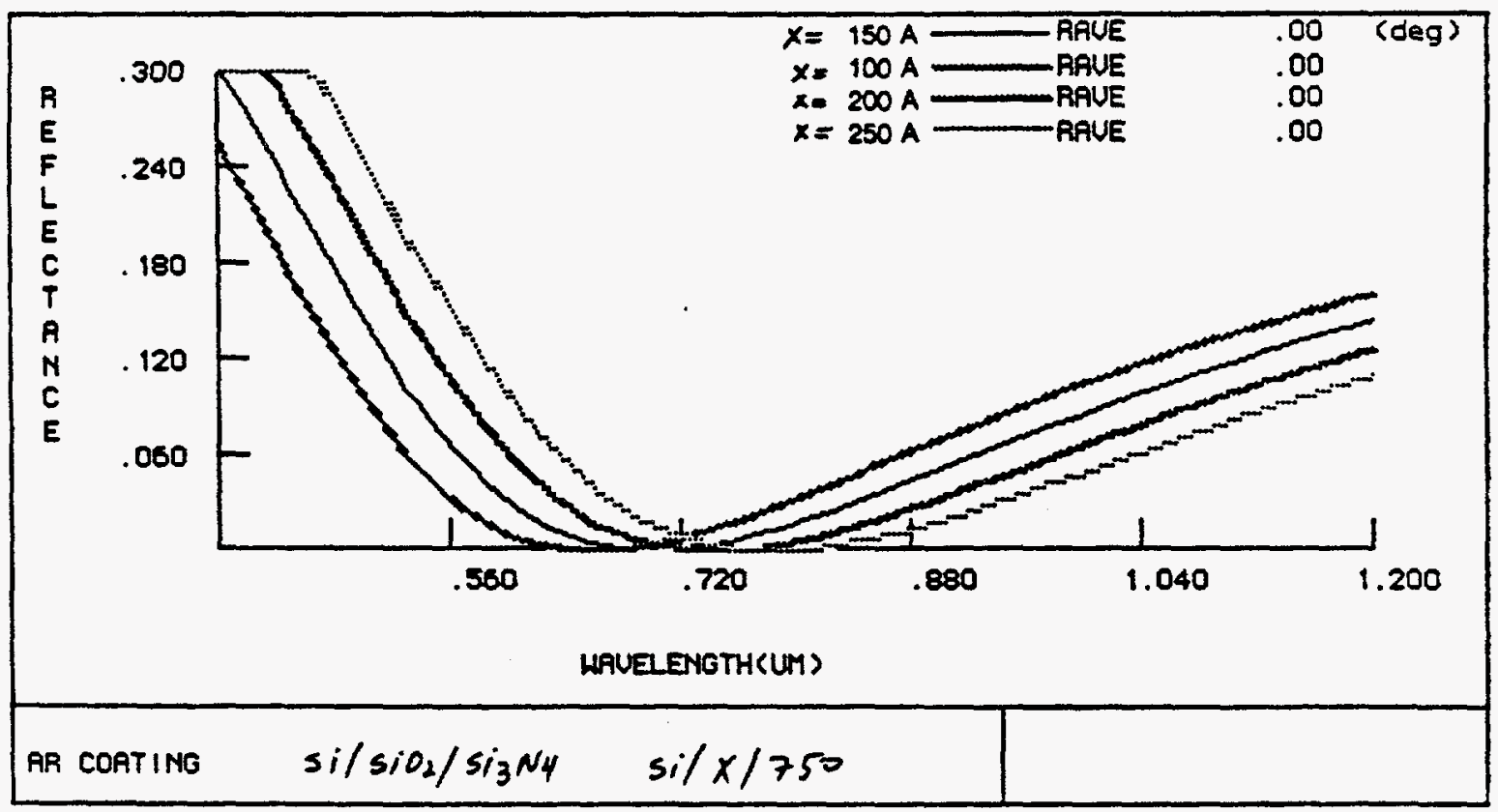

Figure 5-13. Keeping Si3N4 thickness constant and changing the SiO2 thickness to check the effect on the reflectance.

Si3N4 thickness: $750 \mathrm{~A}$

SiO2 thickness: $\times A$ 


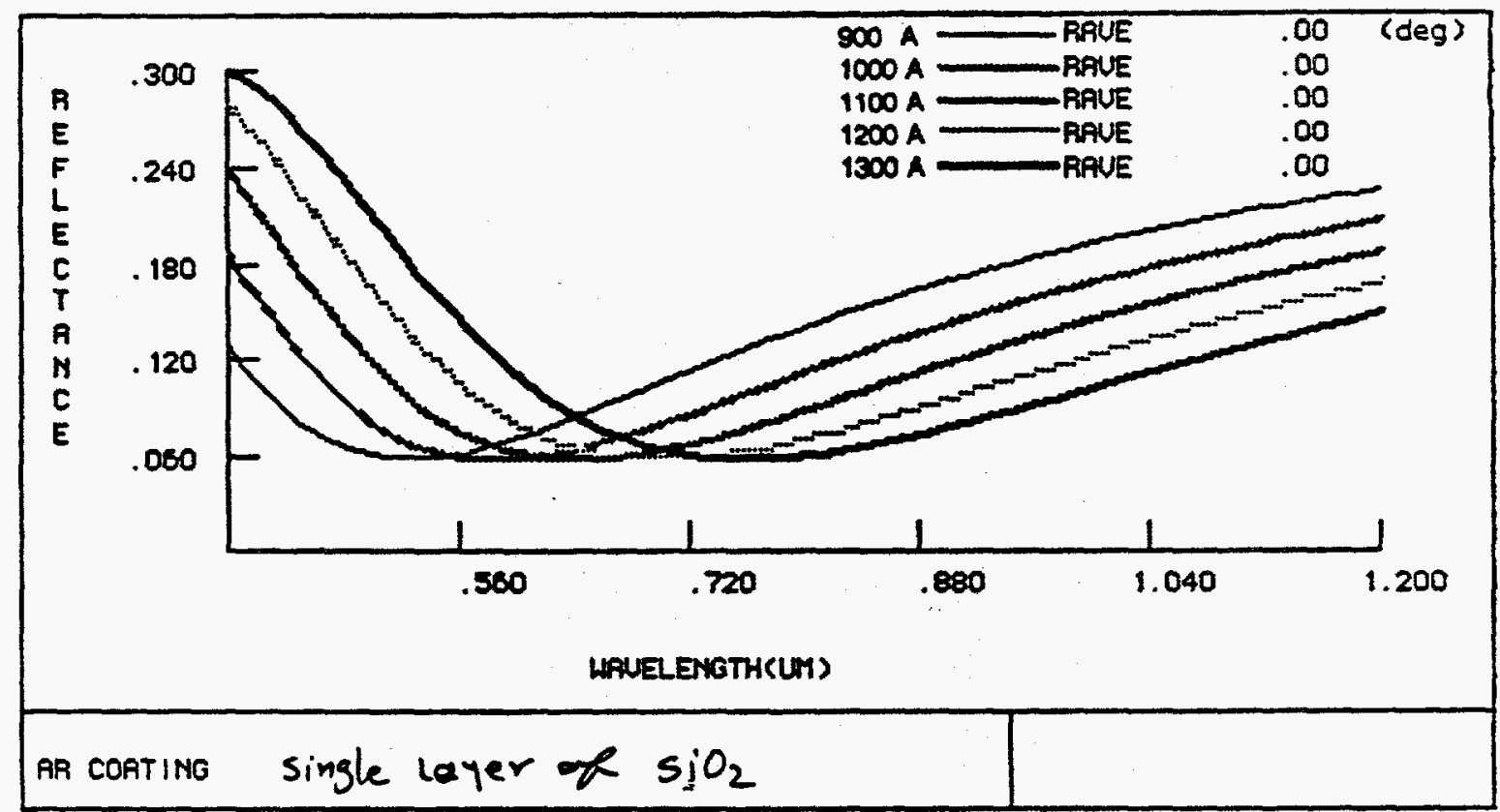

Figure 5-14. Changing the SiO2 thickness to check the effect on the reflectance.

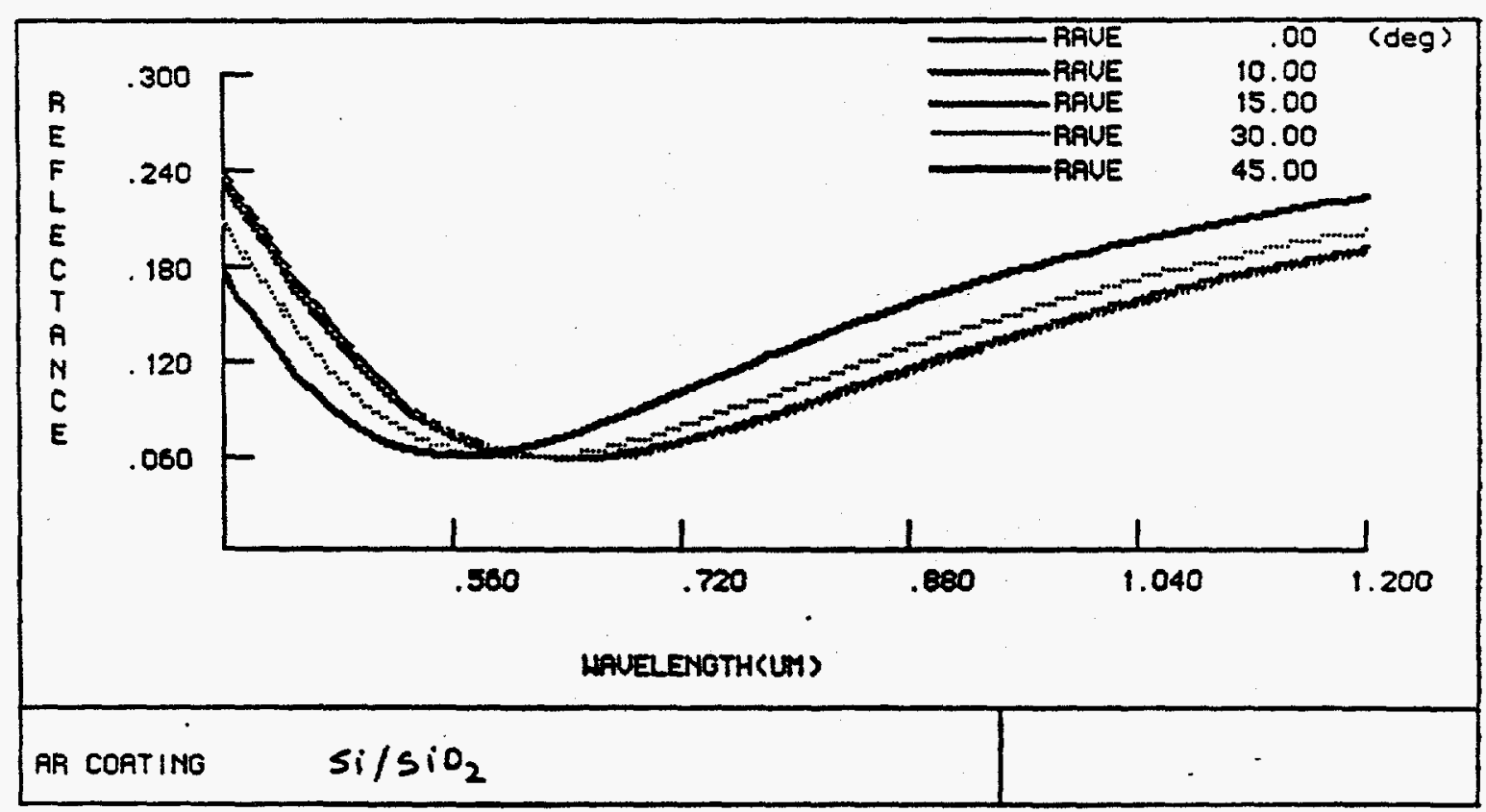

Figure 5-15. Changing the incident angle to check the effect on the refiectance.

SiO2 thickness: $1100 \mathrm{~A}$ 


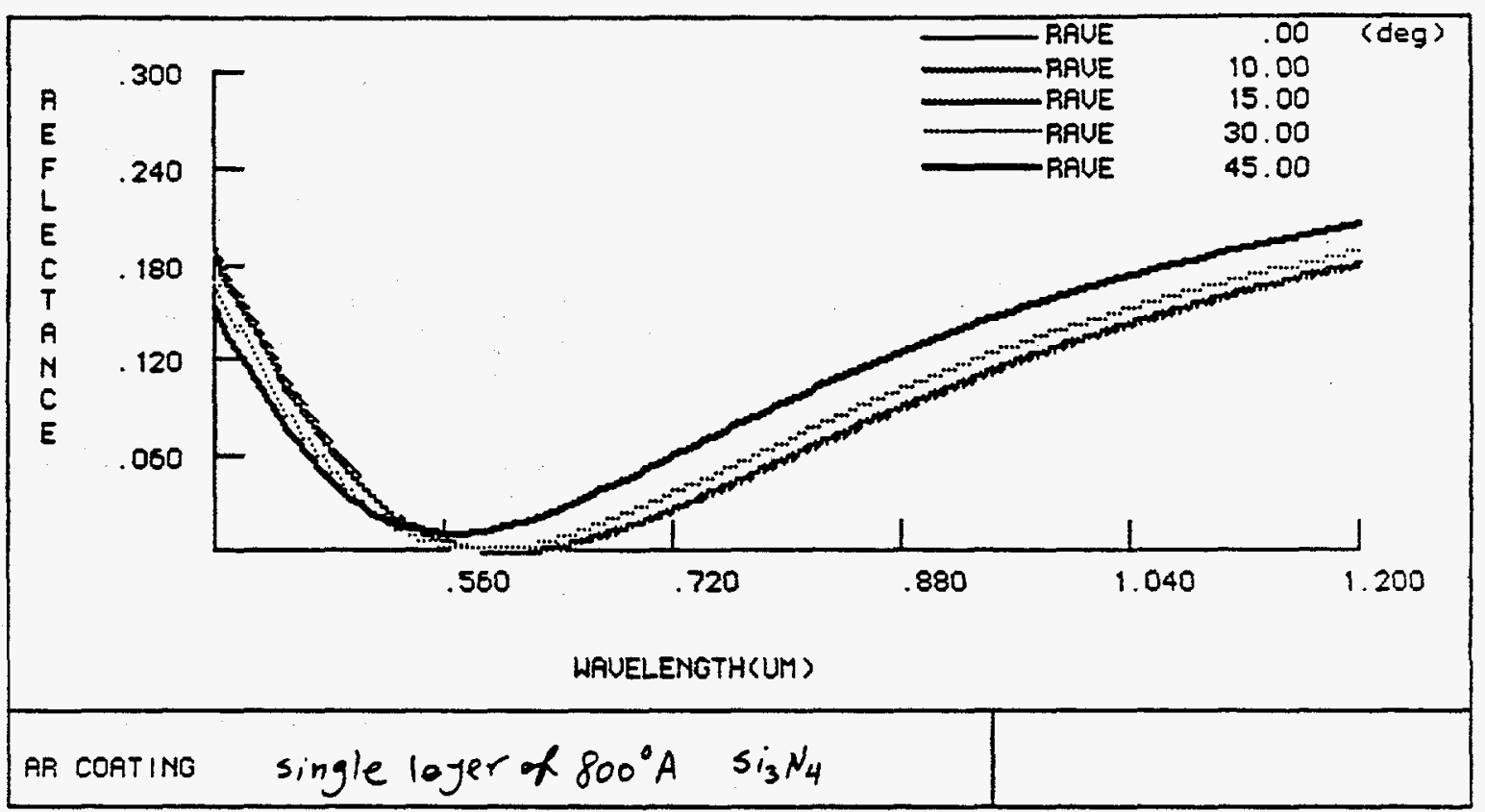

Figure 5-16. Changing the incident angle to check the effect on the reflectance.

Si3N4 thickness: $800 \mathrm{~A}$

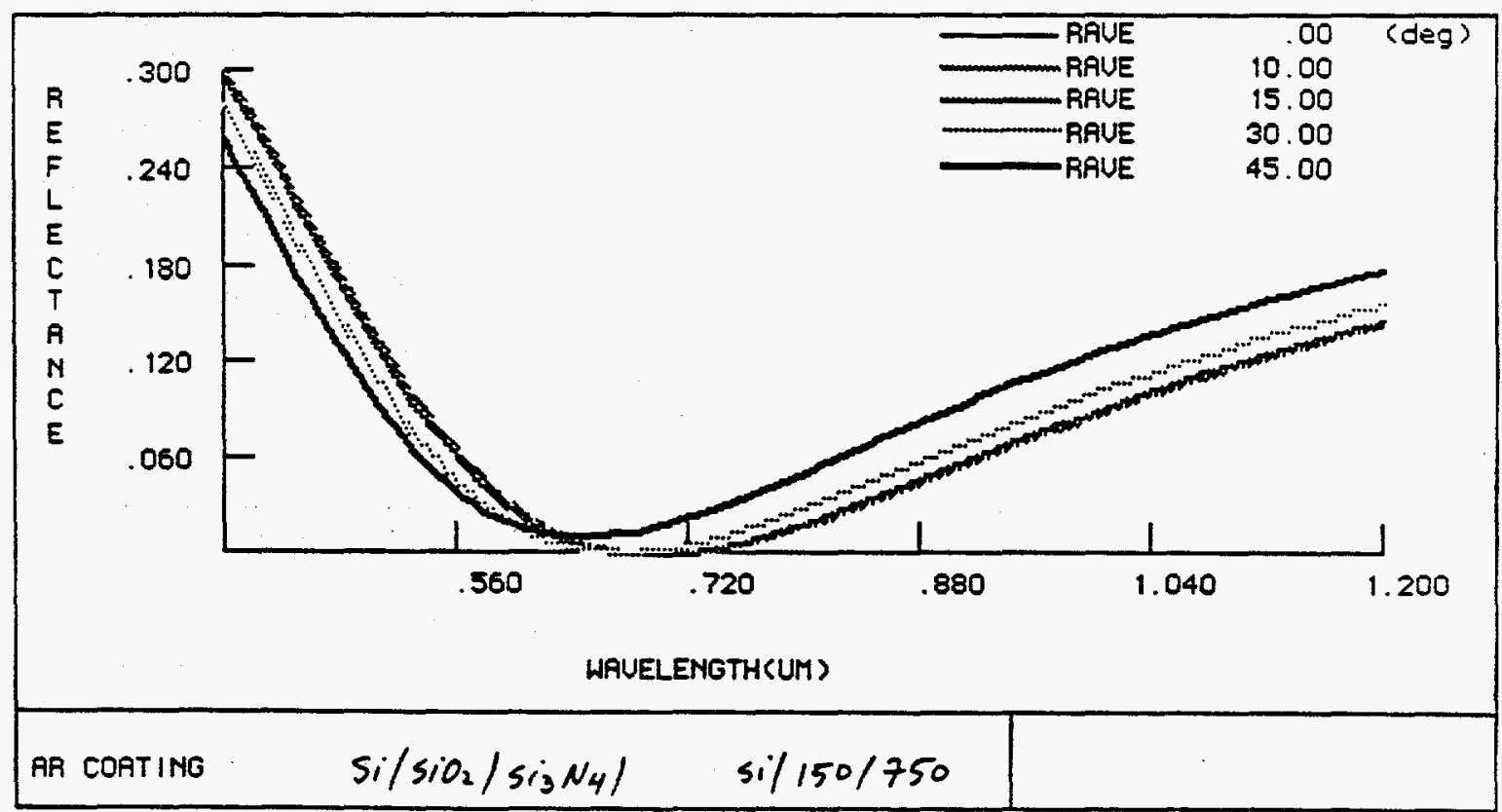

Figure 5-17. Changing the incident angle to check the effect on the reflectance.

SiO2 thickness: $150 \mathrm{~A}$

SiN4 thickness: $750 \mathrm{~A}$ 


\subsection{SOLAR CELL FABRICATION AND RESULTS}

Solar cells were fabricated on .2 ohm-cm p-type silicon wafers to develop a baseline process sequence that can give high efficiency solar cells. First, $\mathrm{N}^{+} / \mathrm{P} / \mathrm{P}^{+}$solar cells were fabricated with $100^{\circ}$ A passivating oxide and no AR coating (Figure 5-18). The following process sequence was used which consists of one oxidation step, two phosphorus diffusion steps ,and one Al sintering step. This process gave $12-13 \%$ efficient solar cells on .2 ohm-cm material without the AR coating.

1. Initial wafer clean

2. Dry oxidation at $1050^{\circ} \mathrm{C}$ for 3 hours

3. Photolithography step to open grid pattern for phosphorous diffusion

4. Deep phosphorous diffusion at $930^{\circ} \mathrm{C}$ for 30 minutes; $20 \Omega / \square$

5. Photolithography to open windows for phosphorous diffusion

6. Phosphorous diffusion at $900^{\circ} \mathrm{C}$ for 15 minutes; $100-150 \Omega / \square$

7. $100 \mathrm{~A}^{\circ}$ passivating oxide at $875^{\circ} \mathrm{C}$ for 10 minutes

8. Photolithography to open rear contact windows

9. Evaporate 1-um Al over the entire rear surface

10. Sinter $\mathrm{Al}$ at $600^{\circ} \mathrm{C}$ in forming gas for 30 minutes

11. Evaporate $\mathrm{Ti} / \mathrm{Ag}$ on front and define grid pattern by lift-off process

12. Silver plate grid to 2 um

13. Forming gas anneal at $450^{\circ} \mathrm{C}$ for 30 minutes

Solar cells were fabricated on low-resistivity $F Z, M C Z$, and $C Z$ silicon. The cell data showed that the difference between the MCZ and FZ cell efficiency is less than $1 \%$, but the $\mathrm{CZ}$ cells gave about $2-3 \%$ lower efficiencies than FZ cells. Lifetime measurements, performed before and after processing, showed that process induced lifetime degradation 


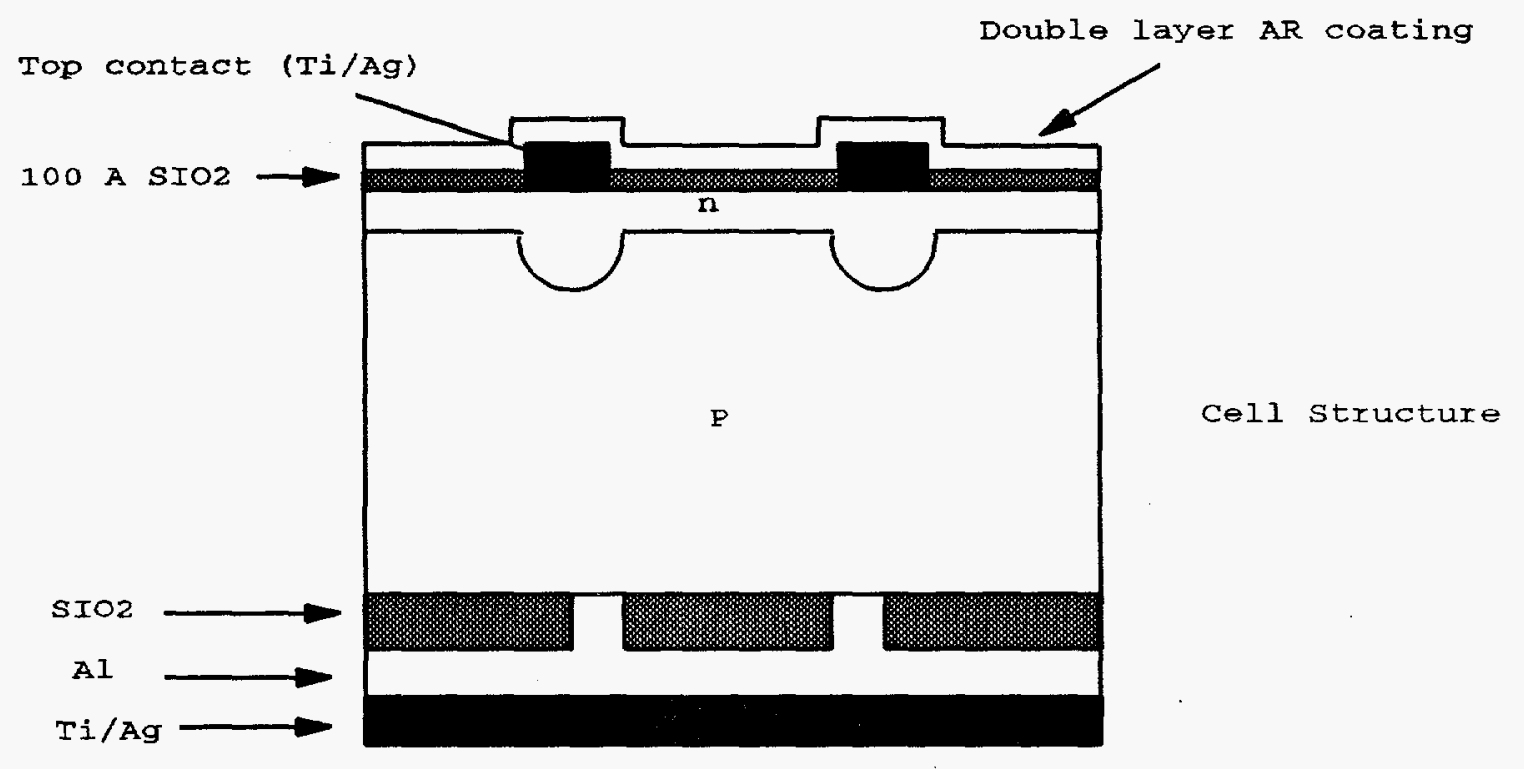

Figure 5-2 . Schematic diagram of the silicon Solar Cell Fabricated at Georgia Tech 
in $\mathrm{CZ}$ material is responsible for lower $\mathrm{CZ}$ cell efficiencies. Tables 5-1, 5-3 and 5-4 show data for the uncoated cells fabricated at Georgia Tech and measured at Sandia National Laboratories. Table 5-2 shows the measured carrier lifetime in the as-grown wafers and finished $F Z, M C Z$, and $C Z$ cells. Cell efficiencies are consistent with the measured lifetimes. CZ silicon shows considerable amount of process-induced lifetime degradation.

Table 5-1. SOLAR CELLS FABRICATED ON FZ MATERIAL

\begin{tabular}{|c|c|c|c|c|}
\hline Cell ID & $\mathrm{J}_{\mathrm{sc}}$ & $\mathrm{V}_{\mathrm{oc}}$ & $\mathrm{FF}$ & EFF\% \\
\hline $1 F Z 1-19$ & 24.90 & .648 & .817 & 13.19 \\
\hline $1 F Z 1-2$ & 25.10 & .649 & .802 & 13.09 \\
\hline $1 F Z 1-7$ & 24.90 & .647 & .811 & 13.04 \\
\hline $1 F Z 1-5$ & 25.00 & .648 & .802 & 12.98 \\
\hline $1 F Z 1-6$ & 24.80 & .644 & .808 & 12.90 \\
\hline $1 F Z 1-12$ & 24.60 & .646 & .813 & 12.88 \\
\hline $1 F Z 1-13$ & 24.20 & .646 & .815 & 12.76 \\
\hline $1 F Z 1-14$ & 24.00 & .647 & .819 & 12.72 \\
\hline
\end{tabular}


Table 5-2. Carrier lifetime in As-grown wafers and finished solar cells fabricated on .2-.3 ohm-cm FZ, MCZ, CZ silicon

\begin{tabular}{||c|c|c|c|c|}
\hline $\begin{array}{c}\text { sample } \\
\text { ID }\end{array}$ & $\begin{array}{c}{\left[\mathrm{O}_{\mathrm{i}}\right]} \\
(\mathrm{ppma})\end{array}$ & $\begin{array}{c}\text { Doping Conc. } \\
\left(\mathrm{cm}^{-3}\right)\end{array}$ & $\begin{array}{c}\text { As-Grown } \\
\tau \text { (us) }\end{array}$ & $\begin{array}{c}\text { Processed } \\
\tau \text { (us) }\end{array}$ \\
\hline PO2FZ & 0.00 & $6.8 \times 10^{16}$ & 175 & 85 \\
\hline PO2MCZ & 10.70 & $6.9 \times 10^{16}$ & 152 & 48 \\
\hline PO2CZ & 15.50 & $7.50 \times 10^{16}$ & 124 & 20 \\
\hline
\end{tabular}

Table 5-3. SOLAR CELLS FABRICATED ON MCZ MATERIAL

\begin{tabular}{|c|c|c|c|c|}
\hline Cell ID & $J_{s c}$ & $V_{\text {oc }}$ & FF & EFF\% \\
\hline$M C Z-35$ & 24.62 & .637 & .805 & 12.63 \\
\hline$M C Z-11$ & 24.58 & .641 & .798 & 12.57 \\
\hline$M C Z-43$ & 24.08 & .635 & .817 & 12.50 \\
\hline$M C Z-41$ & 24.25 & .637 & .803 & 12.40 \\
\hline$M C Z-25$ & 24.03 & .636 & .809 & 12.37 \\
\hline$M C Z-33$ & 23.78 & .635 & .816 & 12.31 \\
\hline$M C Z-24$ & 23.75 & .638 & .811 & 12.30 \\
\hline$M C Z-44$ & 24.02 & .635 & .805 & 12.28 \\
\hline
\end{tabular}


Table 5-4. SOLAR CELLS FABRICATED ON CZ MATERIAL

\begin{tabular}{|c|c|c|c|c||}
\hline Cell ID & $\mathrm{J}_{\mathrm{sc}}$ & $\mathrm{V}_{\mathrm{oc}}$ & $\mathrm{FF}$ & $\mathrm{EFF}$ \\
\hline $\mathrm{CZ}-14$ & 22.46 & .629 & .780 & 11.51 \\
\hline $\mathrm{CZ}-23$ & 23.21 & .626 & .784 & 11.40 \\
\hline $\mathrm{CZ}-22$ & 22.37 & .628 & .809 & 11.36 \\
\hline $\mathrm{CZ}-35$ & 23.50 & .625 & .783 & 11.33 \\
\hline $\mathrm{CZ}-21$ & 22.23 & .628 & .809 & 11.29 \\
\hline $\mathrm{CZ}-32$ & 22.42 & .625 & .798 & 11.19 \\
\hline $\mathrm{CZ}-13$ & 22.06 & .630 & .805 & 11.18 \\
\hline $\mathrm{CZ}-24$ & 21.81 & .626 & .810 & 11.05 \\
\hline
\end{tabular}

As discussed in section 5.2, two layer $\mathrm{ZnS} / \mathrm{MgF}_{2} \mathrm{AR}$ coating was designed and developed. FZ silicon cells were fabricated with the two layer AR coating, Table 5-5. Preliminary attempts have been made to fabricate textured cells with single-layer $\mathrm{SiO}_{2}$ and $\mathrm{Si}_{3} \mathrm{~N}_{4}$ AR coatings. These results are shown in Tables 5-6 and 5-7, respectively. Efficiencies as high as $17.8 \%$ have been achieved so far. More work is in progress to improve the cell efficiencies beyond $20 \%$. 
Table 5-5. .2 ohm-cm FZ with double layer AR

\begin{tabular}{|c|c|c|c|c|}
\hline \hline sample ID & $J_{s c}$ & $V_{o c}$ & $F F$ & EFF\% \\
\hline $10 F Z 12$ & 33.97 & .645 & .775 & 16.99 \\
\hline $10 F Z 13$ & 34.16 & .645 & .785 & 17.30 \\
\hline $10 F Z 23$ & 34.35 & .645 & .792 & 17.59 \\
\hline $10 F Z 33$ & 34.64 & .645 & .791 & 17.69 \\
\hline $10 F Z 34$ & 34.50 & .644 & .781 & 17.36 \\
\hline
\end{tabular}

Table 5-6. $.2 \mathrm{ohm}-\mathrm{cm} \mathrm{FZ}$ textured with $1000 \mathrm{~A}^{\circ} \mathrm{SiO}_{2}$

\begin{tabular}{|c|c|c|c|c|}
\hline sample ID & $J_{s c}$ & $V_{o c}$ & FF & EFF \% \\
\hline 1BPT-02 & 32.70 & .645 & .796 & 16.78 \\
\hline 1BPT-13 & 31.30 & .645 & .812 & 16.41 \\
\hline 1BPT-15 & 34.80 & .635 & .733 & 16.18 \\
\hline 1BPT-12 & 30.90 & .645 & .809 & 16.11 \\
\hline
\end{tabular}

Table 5-7. $.2 \mathrm{ohm}-\mathrm{cm} \mathrm{FZ}$ textured with single layer AR $\left(750 \mathrm{~A}^{\circ} \mathrm{Si}_{3} \mathrm{~N}_{4}\right)$

\begin{tabular}{||c|c|c|c|c||}
\hline sample ID & $J_{s c}$ & $V_{o c}$ & $F F$ & EFF \% \\
\hline FZ2-51 & 35.71 & .629 & .792 & 17.80 \\
\hline FZ2-52 & 34.61 & .631 & .796 & 17.38 \\
\hline FZ2-53 & 34.65 & .628 & .798 & 17.35 \\
\hline FZ2-54 & 34.67 & .633 & .774 & 17.00 \\
\hline
\end{tabular}




\section{APPENDIX A}

The following section is from a paper presented at the $21^{\text {st }}$ IEEE Photovoltaics Specialist Conference. It presents a brief description and som preliminary results of TEXTURE. 


\section{ABSTRACT}

Increased interest in light trapping techniques to enhance the output current of silicon solar cells has led to a variety of surface texturing designs. Unfortunately the inability to quantify and eliminate some of the photon loss mechanisms have not allowed textured cells to reach their full potential. TEXTURE, a monte carlo ray tracing program, has been written which is capable of addressing the above issues. The program has a convenient user interface and a variety of output modes. Different material systems such as GaAs, InP, and thin films on textured glass can also be modelled by Texture. This paper shows that the output of the TEXTURE program not only compares quite favorably with results of other researchers under the same assumptions, but also provides additional quantitative information about photon losses due to the sides of the cell, grid absorption, and imperfect back surface reflectance. Output results show that tetrahedrons are more effective light trapping structures than tilted pyramids $(40.87 \mathrm{~mA}$ compared to $40.37 \mathrm{~mA}$ ).

\section{INTRODUCTION}

Light trapping techniques to enhance the output current of silicon solar cells has led to a variety of surface texturing designs. Methods to quantify the effect of various texturing patterns on different material systems has not been readily available to cell designers. Numerical simulation of light trapping structures would provide an inexpensive resource for the design of high efficiency textured solar cells.

Currently there is no public domain or commercially available ray tracing program 
for the solar cell community. It was recognized that such a program would be extremely helpful in the development of high efficiency cells if it could be applicable to a wide variety of computing capabilities, cell structures, and material systems. In addition the program should provide the user with as many possible loss mechanisms as can be modelled. Texture, a monte carlo ray tracing program, was developed with these and other constraints in mind, Table A-1.

\section{Table A-1. Design attributes of ray tracing program}

1. Applicable to computer system's with Fortran 77 and ANSI (American National Screen Interface)

2. Compatibility with current and future modelling programs

3. New structures easily incorporated due to modular nature of the program

4. 3 dimensional analysis in all modes of operation

5. Variable angle of incidence

6. Surface structures should be independent of each other

7. Different material systems should be easily modelled

8. Cover glass treated as fully as the semiconductor

9. Grid lines modelled as fully as possible

10. Average increase in surface area to be output

11. Various spectra and intensities accounted for

12. Loss of flux due to sides, back, grids, cover glass, and reflection should be provided 
The program was designed to be a companion to PC-1D version 2 [1]. However, incorporation of effects of current interest (grid lines, cover glass, side effects) provide more information than required by $P C-1 D$ and thus the program has become an independent unit. A user friendly interface provides access to the design options supported by the program. This program can support various surface structures including flat, Lambertian, slats, pyramids, tetrahedrons, and square wells, figure A-1.

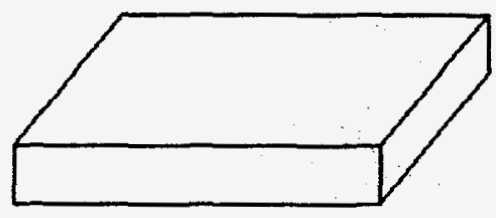

a

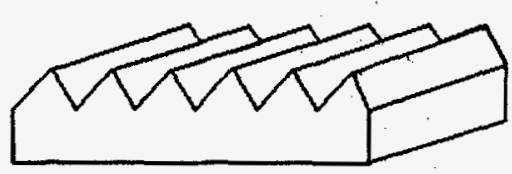

c

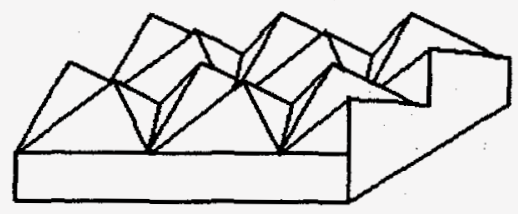

$e$

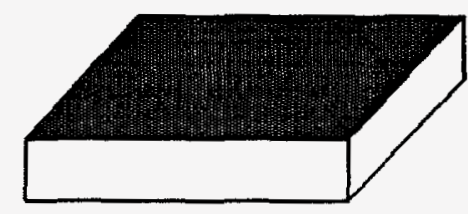

b

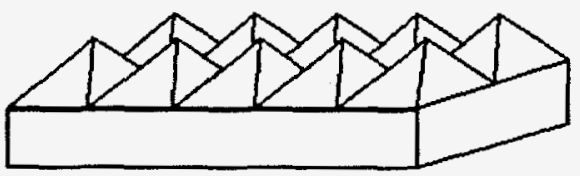

$d$

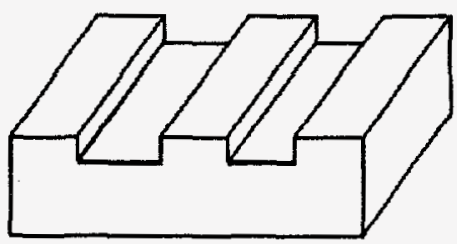

f

Figure A-1. Texturing options supported by the program: a) flat b) Lambertian c) slats d) pyramids e) tetrahedrons f) square wells.

The modular nature of the program allows for the incorporation of other surface features. Other options accounted for by the program include textured cover glass, grid lines of 
variable reflectivity, non-normal light incidence, and variable back surface reflectance.

The remainder of this paper will progress along the following guidelines: The numerical equations used in the tracing of rays will be briefly reviewed. Then the input and output options of the program will be discussed. The application of the program to various material systems and surface texturing schemes will be presented and compared to referenced data. Finally, the creation and coupling of PC-1D compatible photon generation and area files will be discussed and the results of cell simulations presented.

\title{
NUMERICAL METHOD
}

The basis for the numerical method lies in three mathematical concepts. The first is the intersection of a line and plane, to find where the ray strikes a surface. The second mathematical equation is Snell's law of refraction, to find the direction the ray is travelling upon entering the material. Finally, the last mathematical expression will deal with the angle of reflection from a surface. Each of these will be outlined below.

\author{
Intersection of a Ray and Plane
}

The equation for a ray is given by:

$$
\frac{x 1-x 0}{\text { calf }}=\frac{y 1-y 0}{\text { cbet }}=\frac{z 1-z 0}{\text { cgam }}
$$


where $\mathrm{X} 1, \mathrm{Y} 1$, and $\mathrm{Z} 1$ are the coordinates of the point of intersection, $\mathrm{X} 0, \mathrm{Y} 0$, and $\mathrm{Z} 0$ are the coordinates of the initial point. The direction cosines calf, cbet, and cgam are found from either Snell's law or the law of reflection. The equation for a general plane is given by, figure 2-18:

$$
\frac{x 1}{a}+\frac{y 1}{b}+\frac{z 1}{c}=d
$$

where $a, b$, and $c$ are the intercepts of the $x, y$, and $z$ axes respectively. The direction cosines of the normal of the plane, relative to the cartesian coordinates, are given as follows:

$$
\begin{aligned}
& \text { calfn }=\frac{a}{\sqrt{a^{2}+b^{2}+c^{2}}} \\
& \text { cbetn }=\frac{b}{\sqrt{a^{2}+b^{2}+c^{2}}} \\
& \text { cgamn }=\frac{c}{\sqrt{a^{2}+b^{2}+c^{2}}}
\end{aligned}
$$

The calculation of these angles will become important for the angles of refraction and reflection. The point of intersection will be given by solving equations $(A-1)$ and $(A-2)$ for one of the unknown coordinates, then the other two coordinates are found from a 
rearrangement of equation (A-1) to save computation time. The new point of intersection is then checked against three criteria: Is it contained on the correct mathematical plane. Is it on a physical plane. Does the point of intersection lie on the textured surface.

\author{
Snell's Law of Refraction
}

Figure A-2 shows an example of Snell's law of refraction. The plane of the paper is called

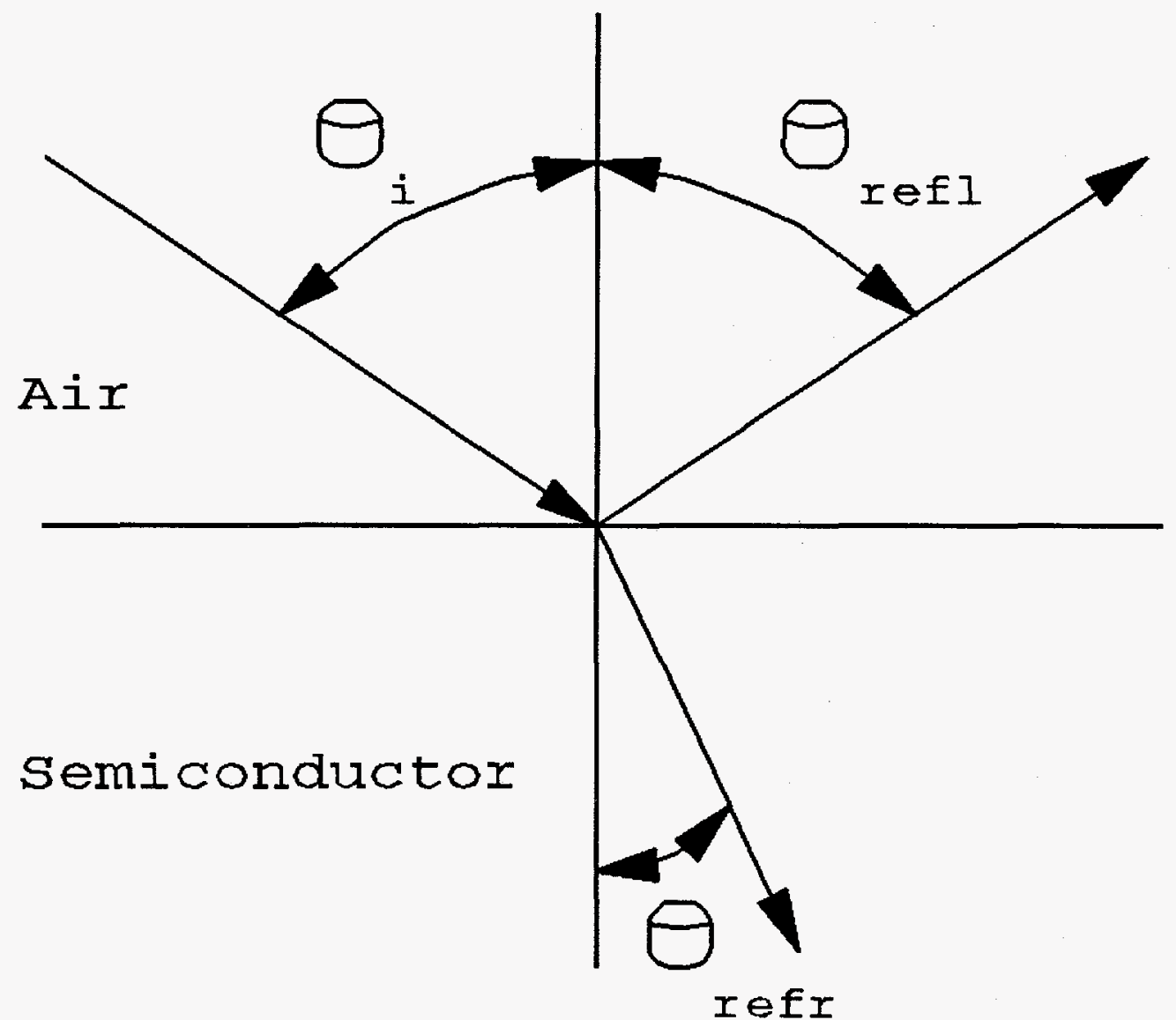

Figure A-2. Snell's law for refraction, reflection ray is also presented.

the refraction (or reflection) plane, and the normal is perpendicular to the incoming ray, 
the refracted ray, and the surface normal. Since the incoming ray and the surface normal direction cosines are known, then the cross product of these two vectors is the refraction plane normal.

$$
\text { refraction plane normal }=\text { incident ray } \otimes \text { surface normal }
$$

Carrying out the vector product leads to the following equations for the direction cosines of the refraction plane normal:

$$
\begin{aligned}
& \text { calfp = cbet } \times \text { cgamn }- \text { cbetn } \times \text { cgam } \\
& \text { cbetp = calfn } \times \text { cgam }- \text { cgamn } \times c a l f \\
& \text { cgamp }=\text { calf } \times \text { cbetn }- \text { calfn } \times \text { cbet }
\end{aligned}
$$

Therefore since the refracted ray is also perpendicular to this plane normal then the dot product of the two vectors will be zero. Mathematically this is equivalent to

$$
0=\text { calfr } \times \text { calfp }+ \text { cbetr } \times \text { cbetp }+ \text { cgamr } \times \text { cgamp }
$$

Where calfr, cbetr, and cgamr are the direction cosines of the refracted ray. The next condition on the refracted ray is given by Snell's law as follows: First the angle between the surface and the incoming ray is found from the dot product of the surface normal and the incoming ray 


$$
\cos \left(\theta_{i}\right)=\text { calf } \times \text { calf }+ \text { cbet } \times \text { cbetn }+ \text { cgam } \times \text { cgamn }
$$

Then by a re-arrangement of Snell's law from

$$
\frac{\sin \left(\theta_{i}\right)}{\sin \left(\theta_{r}\right)}=\frac{\eta_{r}}{\eta_{i}}
$$

to the following form

$$
\cos \left(\theta_{r}\right)=\cos \left(\arcsin \left(\sin \left(\theta_{1}\right) \frac{\eta_{r}}{\eta_{l}}\right)\right)
$$

relates the incoming angle to the cosine of the refracted angle. Yet, this is equivalent to the dot product of the surface normal and the refracted ray

$$
\cos \left(\theta_{r}\right)=\text { calfr } \times \text { calfn }+ \text { cbetr } \times \text { cbetn }+ \text { cgamr } \times \text { cgamn }
$$

The third and final condition will be given by the angle between the refracted ray and the incoming ray. This is given by the sum of the two angles

$$
\cos \left(\theta_{n}\right)=\cos \left(\arccos \left(\theta_{i}\right)+\arccos \left(\theta_{r}\right)\right)
$$

Which is equal to the dot product between the incoming ray and the refracted ray

$$
\cos \left(\theta_{r}\right)=\text { calfr } \times \text { calf }+ \text { cbetr } \times \text { cbet }+ \text { cgamr } \times \text { cgam }
$$

This leads to a three by three system of equations for the unknowns of calfr, cbetr, and cgamr given by 


$$
\left[\begin{array}{ccc}
\text { calfp } & \text { cbetp } & \text { cgamp } \\
\text { calfn } & \text { cbetn } & \text { cgamn } \\
\text { calf } & \text { cbet } & \text { cgam }
\end{array}\right]\left[\begin{array}{c}
\text { calfr } \\
\text { cbetr } \\
\operatorname{cgamr}
\end{array}\right]=\left[\begin{array}{c}
0.0 \\
\cos \left(\theta_{1}\right) \\
\cos \left(\theta_{n}\right)
\end{array}\right]
$$

This system is solved by the method of determinants for the new direction of the refracted ray, calfr, cbetr, and cgamr. These values are then substituted for the old values of the direction cosines (calf, cbet, cgam) and give the new direction the ray is travelling.

\section{Angle of Reflection}

The first condition on the reflected ray is the same as that of the refracted ray, i.e. the ray must be perpendicular to the normal of the plane of reflection. The second condition of the reflected ray is that the angle of incidence is equal to the angle of reflection relative to the surface normal,

$$
\cos \left(\theta_{r}\right)=\cos \left(\theta_{i}\right)
$$

therefore, the following relationship holds, which is the dot product of the reflected ray and the surface normal,

$$
\cos \left(\theta_{i}\right)=\text { calfr } \times \text { calfn }+ \text { cbetr } \times \text { cbetn }+ \text { cgamr } \times \text { cgamn }
$$

The third and final condition on the reflected ray also comes from the fact that the angle of incidence equals the angle of reflection, then the angle between the incident ray and 
the reflected ray is $2 \Theta_{\mathrm{i}}$. The following equation is created by taking the dot product of the reflected ray with the incoming ray,

$$
\cos \left(2 \theta_{i}\right)=\text { calfr } \times \text { calf }+ \text { cbetr } \times c b e t+\operatorname{cgamr} \times c g a m
$$

Arranging these three conditions into a linear set produces

$$
\left[\begin{array}{ccc}
\text { calfp } & \text { cbetp } & \text { cgamp } \\
\text { calfn } & \text { cbetn } & \text { cgamn } \\
\text { calf } & \text { cbet } & \text { cgam }
\end{array}\right]\left[\begin{array}{c}
\text { calfr } \\
\text { cbetr } \\
\text { cgamr }
\end{array}\right]=\left[\begin{array}{c}
0.0 \\
\cos \left(\theta_{i}\right) \\
\cos \left(2 \theta_{i}\right)
\end{array}\right]
$$

Again this system is solved by the method of determinants to produce the new direction cosines of the reflected ray. In addition if the surface being considered is the top of the cell or cover glass and the direction of travel is outward then the angle of incidence is checked against the critical angle. If it is smaller than the critical angle the ray is coupled out of the material, and the new angle of refraction is calculated.

\title{
INPUT AND OUTPUT OPTIONS
}

\author{
Program Input
}

A user friendly interface has been included, if the program is run on an IBM compatible PC. This program creates an input file which is directly read into the main program. The 
first part of the input includes the incident flux, the spectrum file (PC-1D spectrum files are recommended) and the angle of incidence of the light. The next part of the input includes the cell dimensions and the output mode to be used. The output modes will be discussed in the program output section of this paper. The material parameters (absorption coefficients, index of refraction, index of reflection, and back surface reflectance) for the cell may be input as either static values or files with the values of the parameters as a function of wavelength. The top structure of the cell with the corresponding dimensions is the next input variable, then the bottom structure of the cell with its dimensions. In most cases the choices of the top and bottom structure are independent of each other. If a cover glass is present, then the thickness, absorption, index of refraction, index of reflection, and top texture must be input. If grid lines are to be present then the file name which contains the geometry of the lines must be input. Currently only grid lines parallel to the coordinate axes are supported. The top surface and bottom surface reflectivity of the grid can be specified independently, figure A-3.

\section{Program Output}

The output of the program will depend upon which mode of operation was selected by the user. The basic output will contain the cell structure used, with the dimensions, the files or static numbers for material parameters, the spectrum chosen, and the mode dependent data.

If the program were run in the distance mode, then the output contains the percentage of rays remaining as a function of the number of passes through the cell. In addition the total average distance travelled before exiting the cell is included. This value 


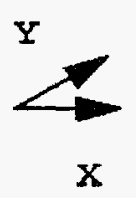

$\mathrm{x}$

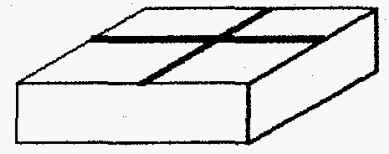

I。
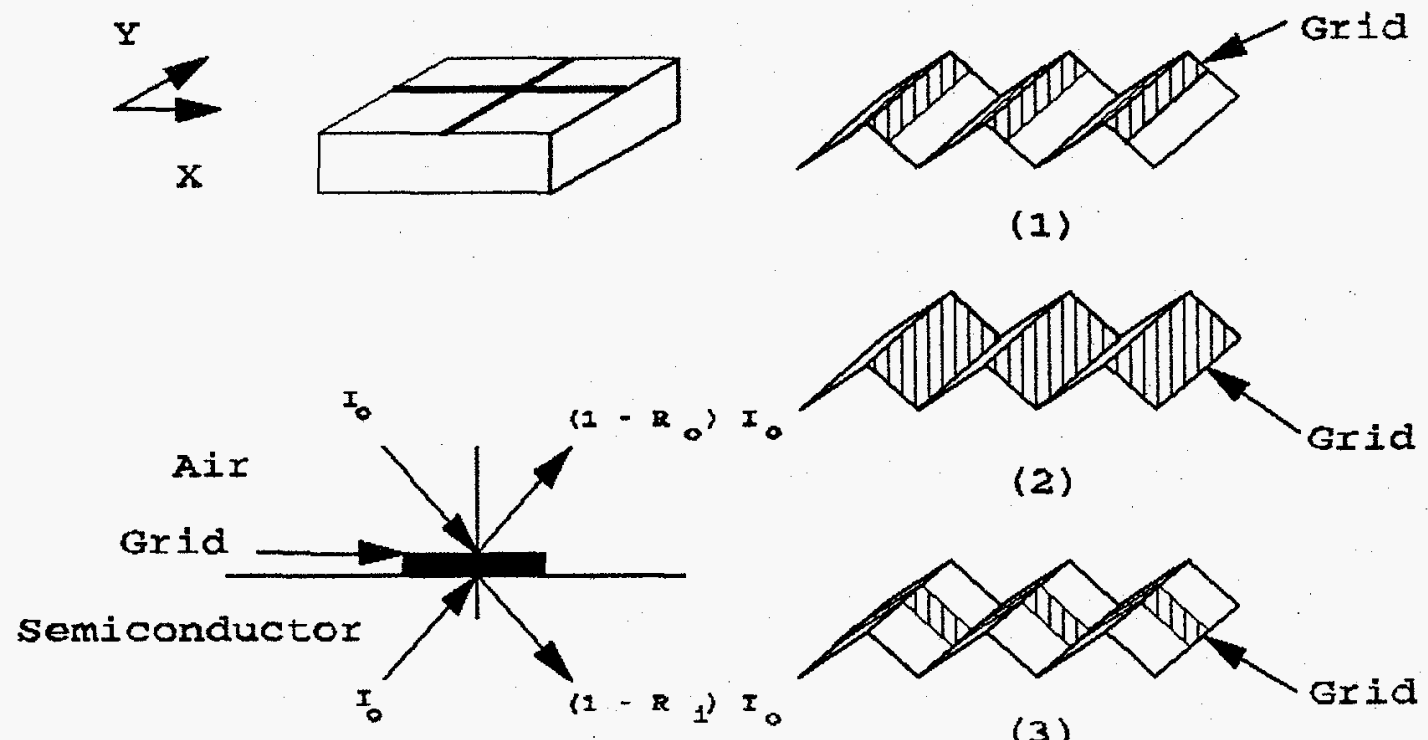

(1)

(3)

Figure A-3. Grid line geometry allowed, reflectivity of top and bottom of grid line. Also depicted are the grid lines used in latter cell simulations

divided by the thickness of the cell gives the relative distance improvement of the cell structure over that of a flat cell of the same dimensions.

Running the program in the absorption mode produces results for the percentage of photons absorbed as a function of wavelength (figure A-4), the total absorbed flux, and the increase in surface area due to the texture. In addition ideal improvements in cell parameters $\left(U_{s c}, V_{o c}\right.$, and $\left.\eta\right)$ are predicted [2]. The cumulative number of photons lost due to grids, sides, and non-ideal back surface reflectance are also included in the output.

One dimensional profile mode produces a cumulative peak to peak generation profile for the structure. The node placement is dictated by a user supplied node file. Again the cumulative losses due to sides, grids, and non-ideal back surface reflectance will be included. 


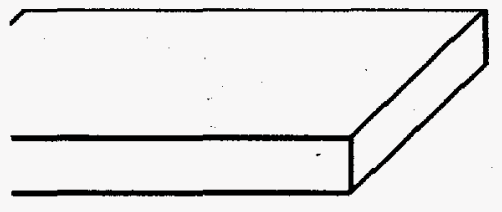

$a$

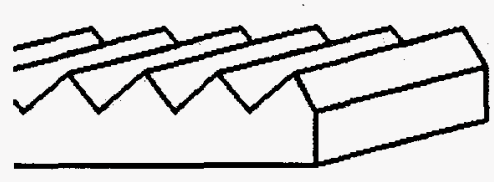

$c$

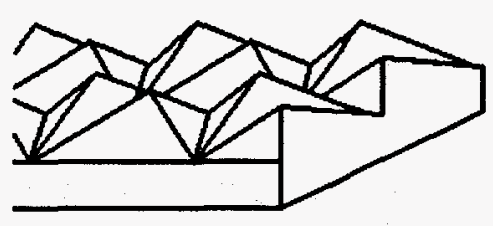

$\Theta$

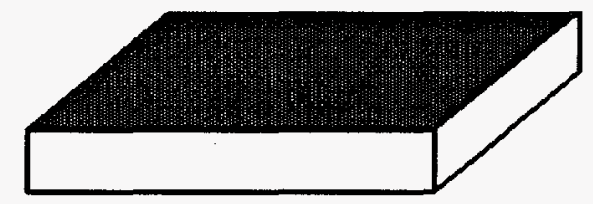

b

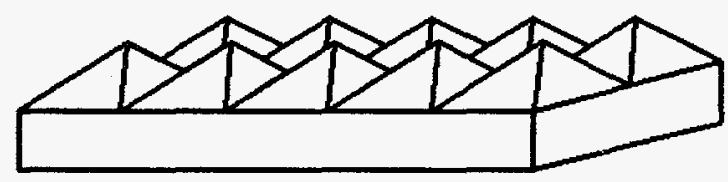

d

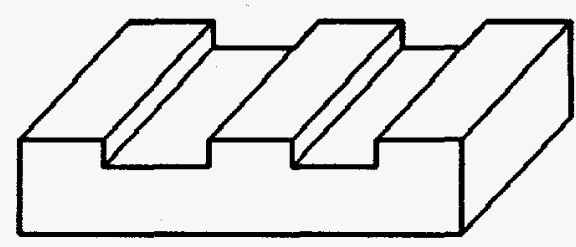

王

Figure A-4. Percentage of photons absorbed as a function of wavelength for a textured cell

In the PC-1D mode, area and one dimensional generation files are created which are directly compatible with PC-1D version 2 (.ARA and .GEN). Again, the node placement is dictated by the user supplied node file. Therefore, one dimensional cell simulations may be attempted. Several examples will be highlighted latter in this paper. A separate file containing the cumulative losses will also be created.

\section{APPLICATION OF PROGRAM}

Table A-2 presents an overview of some results obtained from TEXTURE for silicon, when possible cumulative photon absorption values from other references are listed. Notice 
that TEXTURE provides additional information, the loss of photons due to the sides, nonideal back surface reflectance, and front surface. Although the loss of photons due to the sides of the cell is small at one sun, this can become significant as the cell size decreases or the incident intensity increases. The application of the program to determine the effect of various grid geometries is also presented in Table A-2. In all cases, the front surface was textured with slats, with a flat back. The grid lines were modelled in three configurations (figure A-2) all containing same surface area coverage:

1. symmetrically along the top of slat peak (half the grid line on each side of the peak);

2. all of the grid line running down one face of the slat; 3. running along the contour of the slat.

Table 2-2. Results of simulations by TEXTURE, comparison with referenced data provided.

\begin{tabular}{lccc} 
Structure & TEXTURE & PC-1D & {$[3,4]$} \\
\hline Flat & 36.46 & 36.50 & 37.60 \\
Lambertian & 41.81 & 41.50 & 41.60 \\
Pyramids & 40.11 & - & 39.50 \\
Per. Slats & 42.28 & -- & 42.00 \\
Tilted Pyramids & 40.37 & -- & 41.00 \\
Tetrahedrons & 40.87 & - & -
\end{tabular}

TEXTURE simulations of 100 microns thick cells with $80 \%$ back surface reflector, $5 \%$ front surface reflection.

\begin{tabular}{lllll} 
Structure & Current & Back loss & Side loss & Grid loss \\
\hline Flat & 35.87 & 2.18 & 0.00 & -- \\
$55^{\circ}$ Slats & 38.59 & 2.52 & 0.002 & -- \\
$55^{\circ}$ Slats Grid (1) & 38.67 & 2.55 & 0.044 & 0.037 \\
$55^{\circ}$ Slats Grid (2) & 38.62 & 2.41 & 0.044 & 0.311 \\
$55^{\circ}$ Slats Grid (3) & 37.33 & 2.39 & 0.045 & 0.135 \\
Pyramids & 39.04 & 3.70 & 0.007 & $\ldots-$ \\
Perpendicular Slats & 39.59 & 7.39 & 0.013 & --
\end{tabular}

Another important feature modelled is the effect of a cover glass on a variety of 
cells. Figure A-5 is an example of a cell and cover glass configuration. The encapsulant material is assumed to fill all the space between the glass and textured cell, and possesses the index of refraction and absorption coefficients of the glass. In all of the cases simulated the thickness of the cover glass was $3 \mathrm{~mm}$. In addition, the cover glass absorption coefficient, index of refraction, and front surface reflectance were assumed constant at the values of $0.1 \mathrm{~cm}^{-1}\left(10 \mathrm{~m}^{-1}\right), 1.485$ and $2.5 \%$ respectively. The model can account for various texturing schemes of the cover glass as well as the cell itself.

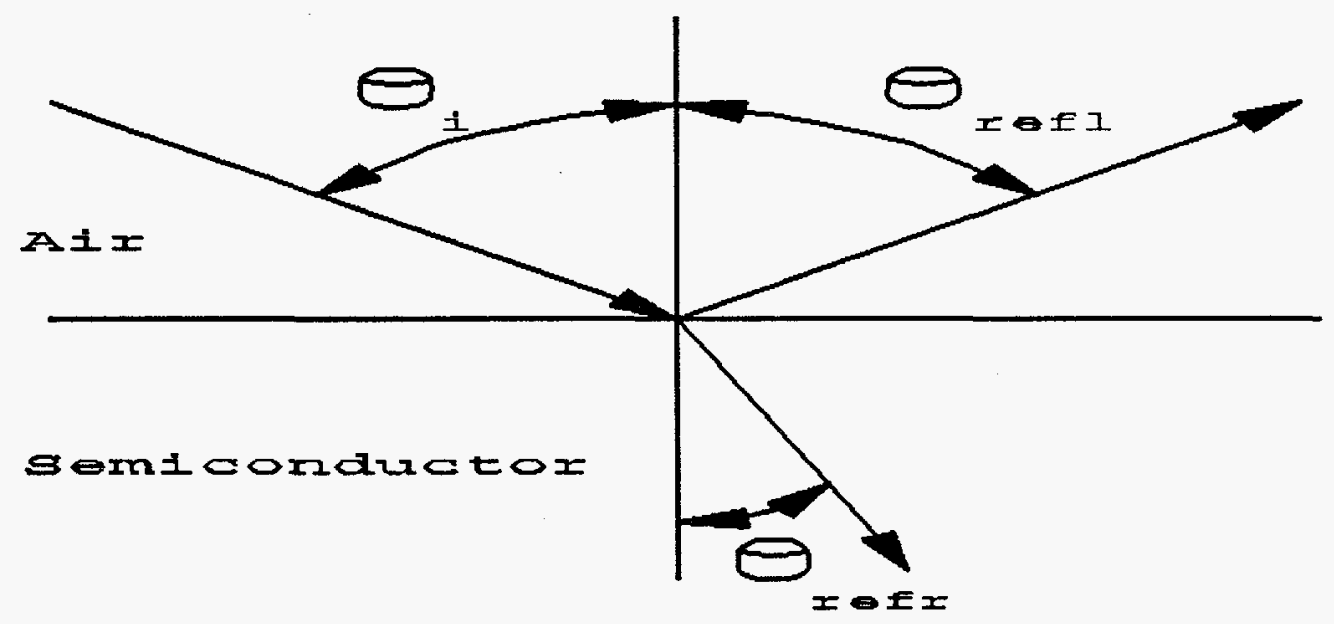

Figure A-5. Textured cell with cover glass

The use of PC-1D compatible absorption coefficient and index of refraction files makes the application of TEXTURE to other material systems quite simple. Table A-3 provides examples of the application of the program to GaAs, InP, and Ge textured cells. When compared to Table A-2, Table A-3 clearly shows that a texturing scheme which provides excellent light trapping for one material may not be as good for another material (Lambertian for silicon compared to Lambertian for GaAs). Also listed in Table 2-3 are simulated systems of textured cover glass for polycrystalline $\|-\mathrm{VI}$ cells (CdTe and 
CulnSe $e_{2}$. In this case the purpose of the simulation was to find the enhancement in absorbed flux. Since the glass is the textured surface the front and back of the $\|-\mathrm{VI}$ material was assumed to be flat, the wide gap window absorption was assumed to be negligible.

Table 2-3. Short circuit current (assuming $100 \%$ collection) for various materials textured in different manners. No back surface reflector except on the Ge based cells ( $80 \%$ effective). Front surface reflectance set to $5 \%$ over all wavelengths.

\begin{tabular}{|c|c|c|c|c|c|}
\hline \multirow{2}{*}{\multicolumn{2}{|c|}{ Material }} & \multirow[b]{2}{*}{ Flat } & \multicolumn{3}{|c|}{ Slats with angles } \\
\hline & & & $45^{\circ}$ & $55^{\circ}$ & Lambertian \\
\hline GaAs & $(6 \mu m)$ & 30.81 & 32.61 & 32.74 & 32.49 \\
\hline $\ln P$ & $(5.5 \mu \mathrm{m})$ & 31.90 & 33.59 & 33.65 & 33.38 \\
\hline $\mathrm{Ge}$ & $(80 \mu \mathrm{m})$ & 56.22 & 59.04 & 59.06 & 58.59 \\
\hline
\end{tabular}

Textured cover glass and II-VI materials

\begin{tabular}{llll} 
Material & Flat & Lambertian & $45^{\circ}$ Slats \\
\hline CulnSe $_{2}$ & 45.18 & 45.30 & 45.71 \\
CdTe & 24.55 & 24.62 & 24.87
\end{tabular}

COUPLING TEXTURE AND PC-1D

In coupling TEXTURE to PC-1D several factors need to be considered. The first is the effective device thickness. This relates how the textured surface height and substrate thickness may be modelled by the quasi-one dimensional simulation of PC-1D. The next is the generation profile which is used over the effective device thickness. It is clear that a peak-to-peak generation profile would only be applicable to the smallest texture height. 
In the determination of the effective device thickness two criteria are used. The first is to try to maintain the total volume of the cell. For example if the front surface is textured in symmetric slats then the volume of the cell is given by the volume of the substrate added to the volume of the peaks which is mathematically

$$
V O L=X D^{*} Y D^{*} T H I C K+F H^{*} F L^{*} Y D^{\star}(X D /(2 F L))
$$

Combining the terms and assuming the area of the substrate to be XD*YD the effective device thickness would be $\left(\right.$ THICK $\left.+0.5^{\star} \mathrm{FH}\right)$. Similar relationships may be found for the other surface texturing geometries. However, if the front texture height is quite large in relation to the length $(F H>>F L)$ then the second criteria becomes important. This second condition states that the device thickness cannot exceed the maximum distance to the nearest point of collection. Figure A-6 will be used in the explanation of this concept. If a photon is absorbed at point $P$, then the length to the nearest point of collection on the front surface is given by

$$
\mathrm{DL}=\left[\left(0.5^{\star} \mathrm{THICK}\right)^{2}+(\mathrm{FL})^{2}\right]^{0.5}+0.5^{\star} \mathrm{THICK}
$$

Therefore the effective device thickness in this case would be given by the minimum of these two conditions

$$
E T H I C K=M I N\left\{D L,\left(T H I C K N E S S+0.5^{\star} F H\right)\right\}
$$



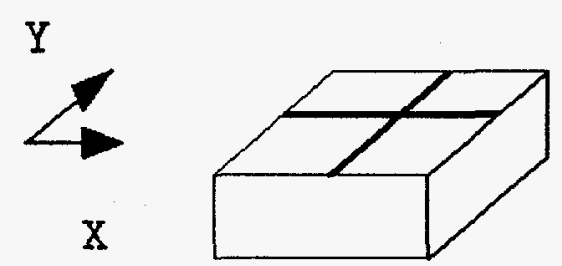
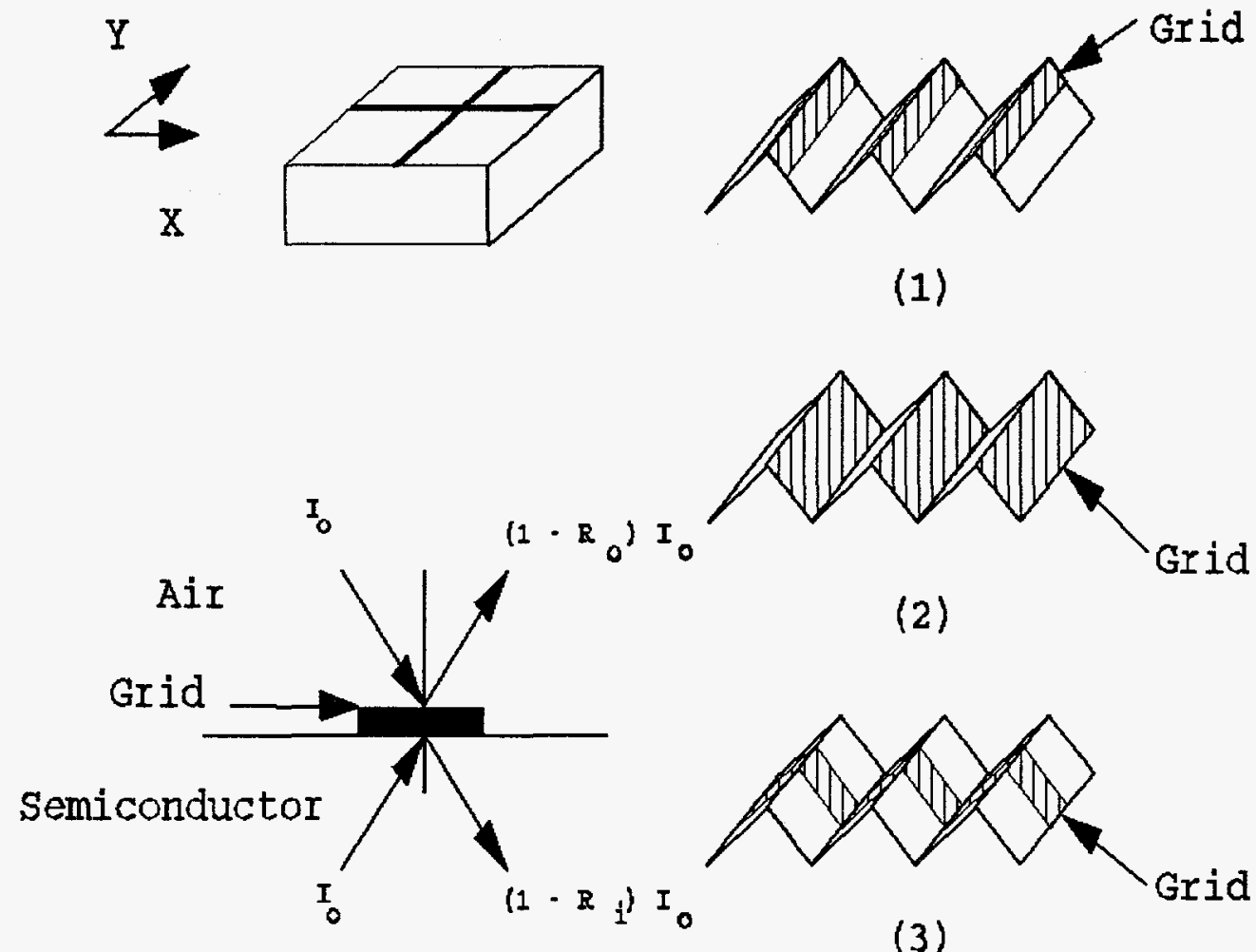

(1)

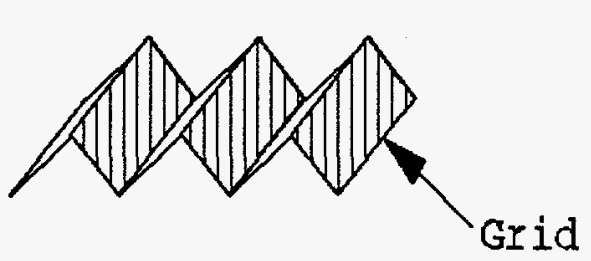

(2)

(3)

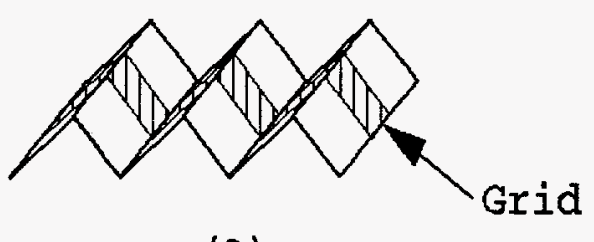

Figure A-6. Concept of maximum distance for collection by a surface for effective device thickness

If the back surface is also textured then the effective device thickness would be given by

$$
\text { ETHICK }=\operatorname{MIN}\left\{D L,\left(T H I C K N E S S+0.5^{*}(F H+B H)\right)\right\} .
$$

Where $\mathrm{DL}$ in this case would include the back texture

$$
\mathrm{DL}=\left[\left(0.5^{\star} \mathrm{THICK}\right)^{2}+(\mathrm{FL})^{2}\right]^{0.5}+\left[\left(0.5^{\star} \mathrm{THICK}\right)^{2}+(\mathrm{BL})^{2}\right]^{0.5}
$$


Similar relations may be formed for other front and back surface texturing geometries. The area file (.ARA) created by TEXTURE for use in PC-1D assumes that the surface area falls off to the area of the substrate in a gaussian manner with the characteristic distance given by one third the effective texture height.

\section{Generation Profile}

In the case of a cell with flat surfaces the generation profile defines how deep within the cell the photon is absorbed. It also reflects the distance from the collecting surfaces.

This concept must be maintained in the transition from the three dimensional generation profile of a textured cell to the one dimensional generation profile of a flat cell for PC-1D. To accomplish the conversion, the minimum distance from the point of absorption to the nearest collecting surface is calculated, either point $A$ or $B$ in figure A-7. The distance on the texture face will be on the line perpendicular to the surface, i.e. along the surface normal. The distance to the bottom of the texture will be given by the pythagorean theorem. By following the path of all of the incoming rays and finding the absorption as a function of this effective depth the one dimensional generation profile for PC-1D (.GEN files) is produced.

Table A-4 is a compilation of several cell simulations carried out using the area and generation files created by TEXTURE for PC-1D, comparison with PC-1D's internal model is provided. 


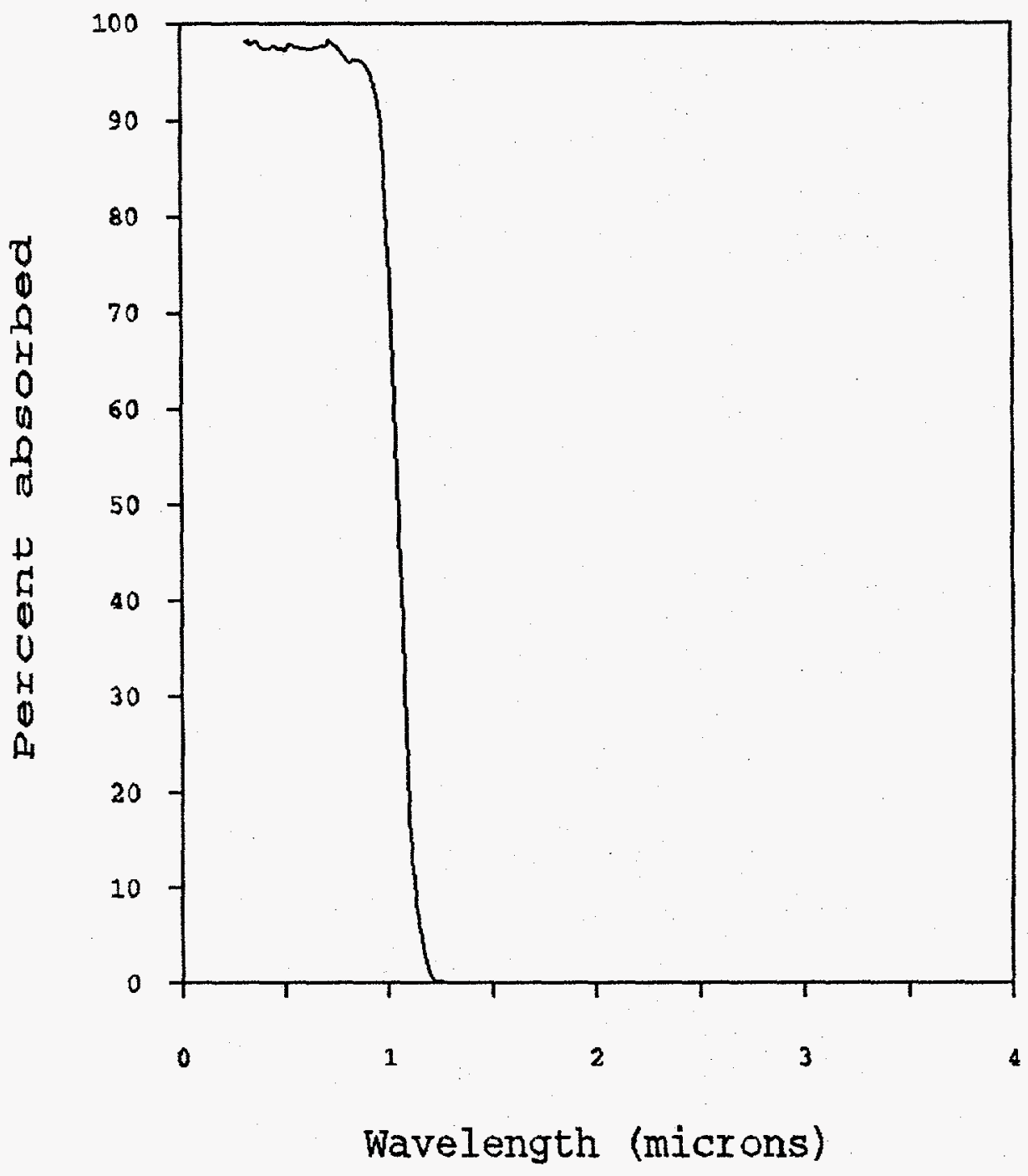

Figure A-7. Nearest distance concept for absorption

Table 2-4. Calculated cell parameters using TEXTURE and PC-1D's internal model for generation and effective area.

\begin{tabular}{llll} 
Structure & Efficiency & $V_{o c}$ & $J_{s c}$ \\
\hline Slats $\left(45^{\circ}\right.$ PC-1D) & 21.13 & 0.691 & 36.34 \\
Slats $\left(45^{\circ}\right.$ TEXTURE) & 21.64 & 0.693 & 37.09 \\
Slats $\left(18^{\circ}\right.$ PC-1D) & 20.17 & 0.690 & 34.76 \\
Slats $\left(18^{\circ}\right.$ TEXTURE) & 20.29 & 0.690 & 34.92
\end{tabular}




\section{REFERENCES}

1. P.A. Basore, PC-1D Installation Manual and User's Guide Version 2.1, lowa State Univeristy Research Foundation, Ames, IA, 1989.

2. R.J. Roedel and P.M. Holm, "The Design of Anisotropically Etched III-V Solar Cells", Solar Cells, vol. 11, pp. 221-239, 1984.

3. M.A. Green and P. Campbell, "Light Trapping Properites of Pyramidally Textured and Grooved Surfaces", in Proceeding of the 19th IEEE Photovoltaic Specialists Conf., pp. 912-917, 1987.

4. P. Campbell, S.R. Wenham, and M.A. Green, "Light Trapping and Reflection Control with Tilted Pyramids and Grooves", in Proceeding of the 20th IEEE Photovoltaic Specialists Conf., pp. 713-716, 1989. 


\section{APPENDIX C.}

\section{ANTIREFLECTION COATING MODEL THEORY}

The main purpose of AR coating is to increase the short-circuit current of the solar cell. Therefore, for optimum design of the cell we must consider the solar spectrum and the quantum efficiency of the cell in the wavelength region of interest. In this report we have considered the airmass 1.5 (AM1.5) spectrum illumination for optimizing the AR coating. In our optimization we have considered wavelength region .3-1.1 um because above $1.1 \mathrm{um}$ the $\mathrm{Si}$ is transparent and therefore no light is absorbed and below $3 \mathrm{um}$ there is negligible energy in the spectrum.

\section{Physics of Single Layer AR Coating}

1. Definitions

- Light propagation is in z-direction

- Quarter-wave thickness: $\lambda=4 n_{i} t_{i}$

- Geometrical thickness: $t_{i}=\lambda / 4 n_{i}$

- Phase: $\phi=2 \pi n_{i} / \lambda$ where $n_{i}$ is the refractive index and $I$ is optical thickness

- Wave number: $k_{i}=2 \pi n_{i} / \lambda \quad\left[\mathrm{cm}^{-1}\right]$

2. Propagation of Light in Isotropic Dielectrics

- Electromagnetic theory combines the material equations, maxwell equations, and the boundary conditions at an interface to give $H_{i}=k_{i} E_{i}$ where $k_{i}=2 \pi n_{i} / \lambda$

- Magnetic and electric fields must be continuous across an interface that contains no free charges.

- At a normal interface 


$$
\begin{gathered}
E_{i}+E_{r}=E_{t} \\
H_{i}-H_{r}=H_{t} \\
n_{i}\left(E_{i}-E_{r}\right)=n_{t} E_{t} \quad\left(n_{i}=n_{r}\right)
\end{gathered}
$$

3. Fields at the Interfaces of a Thin Film Between Two Media

Consider a thin film of thickness $t_{1}$ and refractive index $n_{1}$ shown in Figure $C-1$.

- First Interface

$$
\begin{aligned}
E_{0}+E_{0}^{\prime} & =E_{1}+E_{1}^{\prime} \\
n_{0} E_{0}-n_{0} E_{0}^{\prime} & =n_{1} E_{1}-n_{1} E_{1}^{\prime}
\end{aligned}
$$

- Second Interface

$$
\begin{gathered}
E_{1} e^{i k_{1} t_{1}}+E_{1}^{\prime} \theta^{-1 k_{1} t_{1}}=E_{2} \\
n_{1} E_{1} e^{i k_{1} t_{4}}-n_{1} E_{1}^{\prime} e^{-i k_{1} t_{1}}=n_{2} E_{2}
\end{gathered}
$$

- Define reflectivity $: r=E_{0}^{\prime} / E_{0}$ and transmissivity: $t=E_{2} / E_{0}$

- Solution to the above equations gives

$$
\begin{aligned}
& 1+r=\left[\cos k_{1} t_{1}-i\left(\frac{n_{2}}{n_{1}}\right) \sin k_{1} t_{1}\right] t \\
& n_{0}-n_{0} r=\left[-i n_{1} \sin k_{1} t_{4}+n_{2} \cos k_{1} t_{1}\right] t
\end{aligned}
$$




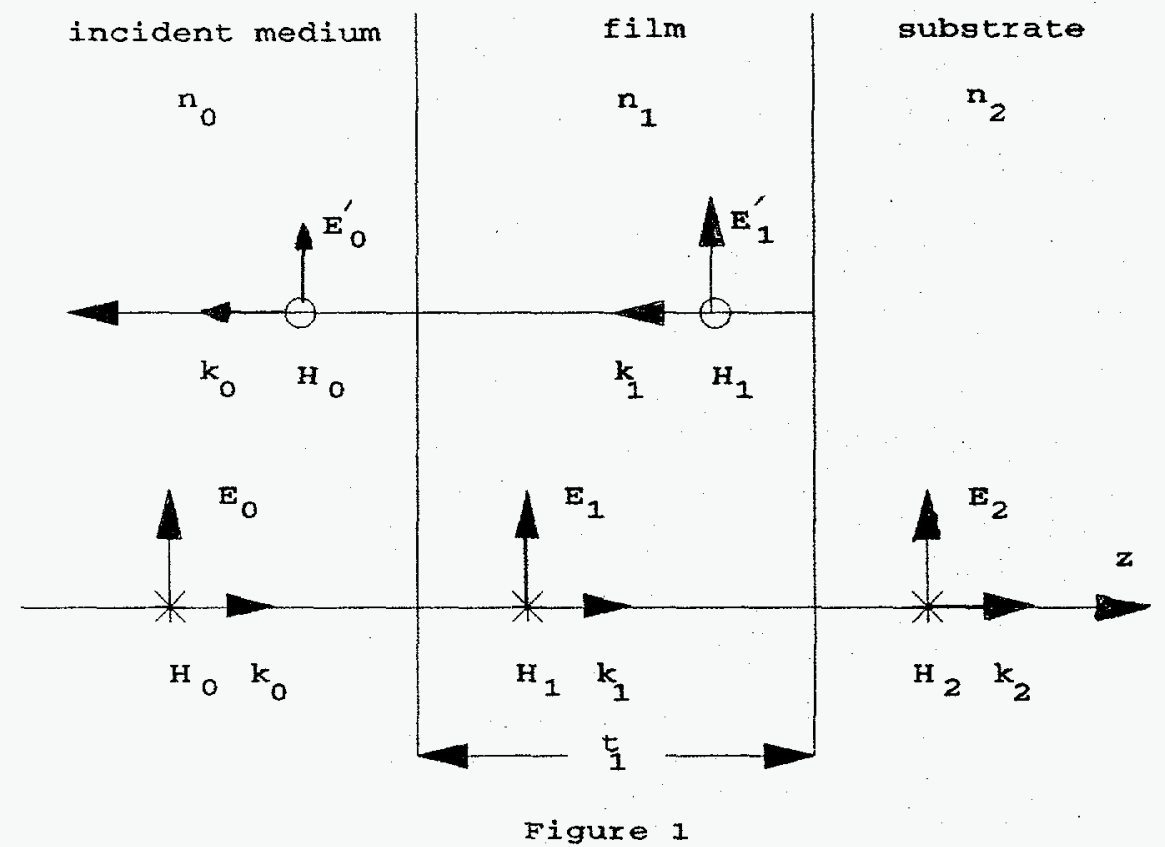

Figure $\mathbf{C}-1$. Fields at the interfaces of a thin film between two media.

- Matrix form for modeling calculations

$$
\begin{gathered}
{\left[\begin{array}{l}
1 \\
n_{0}
\end{array}\right]+\left[\begin{array}{c}
1 \\
-n_{0}
\end{array}\right] r=\left[\begin{array}{cc}
\cos k_{1} t_{1} & -i\left(1 / n_{1}\right) \sin k_{1} t_{1} \\
-i n_{1} \sin k_{1} t_{1} & \cos k_{1} t_{1}
\end{array}\right]\left[\begin{array}{l}
1 \\
n_{2}
\end{array}\right] t} \\
{\left[\begin{array}{l}
1 \\
n_{0}
\end{array}\right]+\left[\begin{array}{c}
1 \\
-n_{0}
\end{array}\right] r=M_{1}\left[\begin{array}{c}
1 \\
n_{2}
\end{array}\right] t}
\end{gathered}
$$

- $M_{1}$ is a matrix that depends only on the parameters of layer 1. 
Where $M_{1}=\left[\begin{array}{cc}\cos _{1} t_{1} & -i\left(1 / n_{1}\right) \sin k_{1} t_{1} \\ -i n_{1} \sin k_{1} t_{1} & \cos k_{1} t_{1}\end{array}\right]$

- Additional layer will contribute other matrices with same index of refraction and thickness dependence, so the generalized form for $P$ intermediate layers is

$$
\begin{gathered}
{\left[\begin{array}{c}
1 \\
n_{0}
\end{array}\right]+\left[\begin{array}{c}
1 \\
-n_{0}
\end{array}\right] r=M_{1} * M_{2} * M_{3} * \ldots . . M_{p} *\left[\begin{array}{c}
1 \\
n_{p+1}
\end{array}\right] t} \\
n_{p+1}=n_{\text {substrate }}
\end{gathered}
$$

- Once matrix multiplication is performed on the intermediate layers there is one $2 \times 2$ matrix $M$ :

$$
M=\left[\begin{array}{cc}
A & -i B \\
-i C & D
\end{array}\right]
$$

- Solving the above for reflectance and transmittance gives

$$
R=|r|^{2}=r \odot r^{*}=\frac{\left(n_{0} A-N_{p+1} D\right)^{2}+\left(n_{0} n_{p+1} B-C\right)^{2}}{\left(n_{0} A+n_{p+1} D\right)^{2}+\left(n_{0} n_{p+1} B+C\right)^{2}}
$$

$$
T=\frac{n_{p+1}}{n_{0}} t \odot t^{*}=\frac{4 n_{0} n_{p+1}}{\left(n_{0} A+n_{p+1} D\right)^{2}+\left(n_{0} n_{p+1} B+C\right)^{2}}
$$

C-4 


$$
R+T=1
$$

- The theoretical calculation to minimize reflectance over the desired wavelengths using the above equations for double layers of $\mathrm{MgF}_{2}$ and $\mathrm{Zns}$ on top of $100^{\circ} \mathrm{A}$ $\mathrm{SiO}_{2}$ gives the optimum thickness of about $550^{\circ} \mathrm{A}$ for $\mathrm{ZnS}$ and $1100^{\circ} \mathrm{A}$ for $\mathrm{MgF}_{2}$.

C-5 
Amonix, Inc. (2)

Attn: Vahan Garboushian Sewang Yoon

$3545 \mathrm{~W}$. Lomita Blvd.

Unit A

Torrance, CA 90505

Applied Solar Energy Corp. (3)

Attn: Ken Ling

Frank Ho

Peter Iles

15251 E. Don Julian Rd.

City of Industry, CA 91749

Arizona State University (2)

College of Engineering

Attn: D. K. Schroder Charles E. Backus

Tempe, AZ 85287

Astropower, Inc. (3)

Attn: Allen Barnett

Bob Hall

James Rand

30 Lovett Ave.

Newark, DE 19711

Boeing Electronics Company

Attn: Lewis Fraas

P. O. BoX 24969, MS 9Z-80

Seattle, WA 98124-6269

Brown University

Div. of Engineering

Attn: J. J. Loferski

Providence, RI 02912

Cornell University

Professor D. Ast

Department of Materials Science and Engineering

Bard Hall

Ithaca, NY 14853-1501

Electric Power Research Inst.

Attn: Ed DeMeo

Frank Goodman

Frank Dostelak

P. O. Box 10412

Palo Alto, CA 94303
ENTECH, InC. (3)

Attn: Mark o'Neill

A. J. McDanal

Mark Jackson

P. O. Box 612246

DFW Airport, TX 75261

Georgia Institute of Technology (2

School of Electrical Engineering

Attn: Ajeet Rohatgi

Richard King

Atlanta, GA 30332

Global Photovoltaic Specialists

Attn: Howard Somberg

21525 Parthenia street

Canoga Park, CA 91304

High Intensity Photovoltaics

Attn: Bernard L. Sater

9007 Westlawn Blvd.

Olmsted Falls, OH 44138

Hughes Research Co.

Attn: Robert Loo

S. Kamath

3011 Malibu Canyon Rd.

Malibu, CA 90265

Kopin Corporation

Attn: J.C.C. Fan

Mark Spitzer

Ronald P. Gale

695 Myles Standish Blvd.

Taunton, MA 02780

James Associates

Attn: L. W. James

7329 Meadow Court

Boulder, CO 80301

Mobil Solar Energy Corp.

Attn: Fritz V. Wald

Dick Bell

Juris Kalejs

Jacob Murad

Four Suburban Park Drive Billerica, MA 01821 
NASA/Lewis Research Center (4)

Attn: Vic Weizer

M. F. Piszczor, Jr.

Henry Curtis

Dennis Flood

21000 Brookpark Rd.

Cleveland, OH 44135

National Institute of

Standards and Technology

Attn: Jon C. Geist

Gaithersburg, MD 20899

North Carolina State Univ. Materials Engineering Dept. Attn: George A. Rozgonyi

Raleigh, NC 27650

Oak Ridge National Lab

Attn: R. F. Wood

P. O. BoX $Y$

Oak Ridge, TN 37830

Penn State University

Attn: S. J. Fonash

127 Hammond Building

University Park, PA 16802

Purdue University (3)

Electrical Engineering Dept.

Attn: R. J. Schwartz

Jeff Gray

Mark Lundstrom

West Lafayette, IN 47907

Research Triangle Institute (3)

Attn: James Hutchby

Michael Timmons

R. A. Whisnant

P. O. Box 12194

Research Triangle Park, NC 27709

Rensselaer Polytechnic Inst.

Attn: S. H. Ghandi

J. M. Borrego

Troy, NY 12181
Seimens Solar Industries

Attn: Charles Gay

Ray Kosanke

Kim Mitchell

4650 Adohr Lane

P. O. Box 6032

Camarillo, CA 93010

SERI Library (2)

1536 Cole Blvd. Bldg. \#4

Golden, CO 80401

SOLEC International, Inc.

Attn: Ishaq Shahryar

12533 Chadron Ave.

Hawthorne, CA 90250

Solar Energy Research Inst. (6)

Attn: John Benner

Jack Stone

Cecile Leboeuf

Ted Ciszek

Tom Surek

Bushan Sopori

1617 Cole Boulevard

Golden, CO 80401

Solarex Corporation (3)

Attn: John Wohlgemuth

Mohan Narayanan

Steve Shea

630 Solarex Court

Frederick, MD 21701

Spectrolab, Inc. (2)

Attn: David Lillington

Gerald crotty

12500 Gladstone Ave.

Sylmar, CA 91342

Spire Corporation (3)

Attn: Chris Keavney

Roger Little

steve Tobin

Patriots Park

Bedford, MA 01730

SUNY-Albany

Attn: J. W. Corbett

P. O. Box 9

Albany, NY 12201 
SunPower Corporation

Attn: R. M. Swanson
R. A. Sinton

435 Indio Way, Suite 100

Sunnyvale, CA 94086

Texas Instruments

Attn: Eric S. Graf

13500 Central Exp.

Dallas, TX 75265

University of Florida (2)

Dept. of Electrical Engineering

Attn: A. Neugroschel

F. A. Lindholm

Gainesville, FL 32611

University of New South Wales

Department of Electrical Engr.

(3)

Attn: Martin A. Green

P. O. Box 1

Kensington, New South Wales

Australia 2033

University of Pennsylvania

Department of Electrical Engr.

Attn: Martin Wolf

Philadelphia, PA 19104

University of Washington

Joint Center for Graduate study

Attn: Larry c. Olsen

Richland, WA 99352

U. S. Department of Energy (9)

Attn: Robert $\mathrm{H}$. Annan

Morton B. Prince

James E. Rannels (5)

Lloyd Herwig

Richard King

Independence Avenue, SW

Washington, DC 20585

U. S. Department of Energy

Attn: D. L. Krenz

John Hanson

P. O. Box 5400

Albuquerque, NM 87115
VS Corporation

Attn: Jan Werthen

1054 Saratoga-Sunnyvale Rd.

Suite 110

San Jose, CA 95129

Westinghouse Electric Corp.

P. O. Box 10864

Attn: Charles M. Rose

Dick Hopkins

Pittsburgh, PA 15236

Wright Patterson AFB (2)

AFWAL/POOC

Attn: Jack Geis Steve Cloyd

wright Patterson AFB, OH

1140 P. S. Peercy

1141 H. T. Weaver

1144 D. S. Ginley

1815 G. E. Pike

3141 S. A. Landenberger (5)

3145 Document Processing for DOE/OSTI (8)

3151 G. C. Claycomb

2531

6200

B. H. Rose

B. W. Marshall

6220

6220

6220

6223

6224

6224

6224

6224

6224

8523-2
D. G. Schueler

A. V. Van Arsdall

J. Pineau,

Solar Library (5)

G. J. Jones

D. E. Hasti

P. A. Basore

J. M. Gee

D. S. Ruby

W. K. Schubert 